\title{
MARKOVIAN PERTURBATION, RESPONSE AND FLUCTUATION DISSIPATION THEOREM
}

\author{
AMIR DEMBO* JEAN-DOMINIQUE DEUSCHEL ${ }^{\dagger}$
}

\begin{abstract}
We consider the Fluctuation Dissipation Theorem (FDT) of statistical physics from a mathematical perspective. We formalize the concept of "linear response function" in the general framework of Markov processes. We show that for processes out of equilibrium it depends not only on the given Markov process $X(s)$ but also on the chosen perturbation of it. We characterize the set of all possible response functions for a given Markov process and show that at equilibrium they all satisfy the FDT. That is, if the initial measure $\nu$ is invariant for the given Markov semi-group, then for any pair of times $s<t$ and nice functions $f, g$, the dissipation, that is, the derivative in $s$ of the covariance of $g(X(t))$ and $f(X(s))$ equals the infinitesimal response at time $t$ and direction $g$ to any Markovian perturbation that alters the invariant measure of $X(\cdot)$ in the direction of $f$ at time $s$. The same applies in the so called FDT regime near equilibrium, i.e. in the limit $s \rightarrow \infty$ with $t-s$ fixed, provided $X(s)$ converges in law to an invariant measure for its dynamics. We provide the response function of two generic Markovian perturbations which we then compare and contrast for pure jump processes on a discrete space, for finite dimensional diffusion processes, and for stochastic spin systems.
\end{abstract}

\section{INTRODUCTION AND OUTLINE}

One of the fundamental premises of statistical physics, the Fluctuation Dissipation Theorem (FDT), follows from the assumption that the response of a system in thermodynamic equilibrium to a small external perturbation is the same as its relaxation after a spontaneous fluctuation. The FDT provides an explicit relationship between the equilibrium fluctuation properties of the thermodynamic system and its linear response (e.g. susceptibility), which involve out-of-equilibrium quantities. As such it relates the dissipation of dynamics at thermal equilibrium of molecular scale (i.e. microscopic) models, with observable macroscopic response to external perturbations, allowing the use of microscopic models for predicting material properties (in the context of linear response theory). Among notable special cases of the FDT are the Einstein relation between particle diffusivity and its mobility 8 (or the recent accounts in [11, [19] and 20]), and the Johnson-Nyquist formula [22] for the thermal noise in a resistor.

Date: June 17, 2007; Revised: February 16, 2010.

*Research partially supported by NSF grants \#DMS-0806211, \#DMS-0406042 and \#DMSFRG-0244323.

${ }^{\dagger}$ Research partially supported by DFG grant \#663/2-3.

AMS (2000) Subject Classification: Primary: 60J25, 82C05, Secondary: 82C31, 60J75, $60 \mathrm{~J} 60,60 \mathrm{~K} 35$

Keywords: Markov processes, Out of equilibrium statistical physics, Langevin dynamics, Dirichlet forms, Fluctuation Dissipation Theorem. 
When deriving the FDT in equilibrium statistical physics one typically starts from a measure $\mu^{0}(\cdot)$ which is often a Gibbs measure, characterized by a Hamiltonian $H(\cdot)$, and a dynamics $X(s)$ for which this measure is invariant. For $\delta>0$ small, one perturbs the dynamics so it becomes invariant for the Gibbs measure corresponding to the Hamiltonian $H(\cdot)+\delta f(\cdot)$. The linear response measures the effect of applying such perturbation at time $s$ on the rate of change, as $\delta \downarrow 0$ in the value of a test function $g(\cdot)$ at time $t>s$ (often taking $f=g$ to be the state variable of the dynamics in question). This response function (of $s$ and $t$ ) is then compared to the rate of change in $s$ of the covariance between $f(X(s))$ and $g(X(t))$ at equilibrium, in the non-perturbed dynamics, whereby the FDT states that the ratio between these two functions is merely $\beta$, the inverse of the system's temperature.

Whereas the FDT is well established and understood in physics, at least in or near thermal equilibrium, see [16, 17], our goal here is to provide its rigorous derivation from a mathematical perspective, as a result about perturbations of Markovian semi-groups. This is easy to do in special (simple) cases, most notably, when dealing with a Markov process on a finite state space. Aiming here instead for a unified derivation, we formalize in Definition 2.7 the concept of having a linear response function in the general framework of a family of continuous time, homogeneous Markov processes $X^{f}(\cdot)$ that are invariant for the measures $\mu^{f}(\cdot)=$ $e^{f} \mu^{0}(\cdot)$ (see Assumption 2.5 for the precise setting). In our definition, the response function $R_{X^{f}, g}(s, t)$ depends on the initial position of the process at time 0 , thereby allowing us to study the effect of the initial measure on the FDT relation. Though this function is uniquely defined per family $X^{f}(\cdot)$, it depends not only on the given Markov process $X(\cdot)$ but also on the chosen perturbation $X^{f}(\cdot)$ of it (compare for example Theorems 4.1 and 4.2). In Theorem 2.10 we characterize the set of all possible response functions for a given Markov process $X(\cdot)$ and show that they all satisfy the FDT relation (2.13). It states that if the initial measure $\mu^{0}(\cdot)$ is invariant for the underlying Markov process $X(\cdot)$, then the dissipation, that is the derivative in $s$ of the covariance between $f(X(s))$ and $g(X(t))$ for $s<t$ equals the $\mu^{0}$-average of the infinitesimal response $R_{X^{f}, g}(s, t)$ to any Markovian fluctuation $X^{\delta f}(\cdot)$ that for $\delta \downarrow 0$ alters the invariant measure of $X(\cdot)$ in the direction of test function $f(\cdot)$ at time $s$, as registered at time $t$ via test function $g(\cdot)$. We show in Corollary 2.13 that this FDT relation holds in the limit $s \rightarrow \infty$ and $t-s$ fixed, whenever the initial measure is such that the law of $X(s)$ converges (in the appropriate sense) to an invariant measure $\mu^{0}(\cdot)$ and in Proposition 2.14 we specify the set of all possible response functions in case of $\mu^{f}$-symmetric (i.e. reversible) Markovian perturbations. Note that the FDT relation has to do with invariance of $\mu^{0}(\cdot)$ but does not require the Markov process $X(\cdot)$ to be reversible with respect to $\mu^{0}(\cdot)$. To further demonstrate how widely this theory can be used, we construct in Section 3 two generic families of Markov perturbations satisfying Assumption 2.5. which apply for any Markov process (subject only to mild restrictions on the domain of certain generators). Namely, the time change of Propositions 3.1 and 3.4 and the generalized Langevin dynamics of Proposition 3.2 (in the symmetric case) and Proposition 3.8 (in the general, non-symmetric case). The bulk of the mathematical work in this paper is in proving that these two generic families admit a response function per Definition 2.7. This is done in Theorems 4.1 and 4.2 of Section 4 which also provide an explicit formula for the response function in each case. 
Moving to examples of specific Markov processes, in Proposition 5.1 we use the simple sufficient condition of Proposition 2.9 for the existence of a response function, to show that essentially all choices of the response function that are possible per Theorem 2.10 are indeed attainable in the context of pure jump processes on a discrete state space. We also demonstrate there how to create a host of perturbations via cycle decomposition (for example, perturbation of a Metropolis dynamics, or of a Glauber dynamics). In Section 6 we illustrate our generic Langevin perturbation for diffusion processes on connected, compact, smooth, finite dimensional manifolds without boundary, showing in Proposition 6.1 that it is generated in this case by the addition of a smooth drift, which for symmetric diffusions is of gradient form. Finally, in Section 7 we demonstrate the flexibility of our framework by considering such a perturbation for infinite dimensional diffusion processes associated with stochastic spin systems in the setting of Gibbs distributions.

All our derivations and results apply even when the Markov process $X(\cdot)$ has more than one invariant measure (as is often the case in statistical physics when the system's temperature is sufficiently low), and most of them apply also for nonreversible dynamics (i.e. having non-symmetric semi-groups).

We note in passing that the appearance of $\beta$ in the FDT in physics is merely due to the definition of Gibbs measure as proportional to $e^{-\beta H}$, with $H$ the corresponding Hamiltonian, and not counting $\beta$ as part of the perturbation $\delta f$. It makes more sense for the mathematical version of the FDT to not mention $\beta$ (so it in effect corresponds to doing statistical physics at $\beta=1$ ). Also, though from a mathematical point of view the response function can not be defined solely in terms of the given Markov process $X(\cdot)$, this is never an issue in physics, whereby viewing the (Markov) dynamics as a classical approximation of a quantum system, the perturbation of its Hamiltonian in direction $f(\cdot)$ uniquely defines also the perturbed quantum system dynamics, hence its classical approximation $X^{f}(\cdot)$ (for example, see the physics based derivation in [14] of the FDT in quantum statistical mechanics).

\section{General theory: FDT at or near EQuilibrium}

A continuous time, homogeneous, strong Markov process $X(t)$ with values in a complete, separable metric space $\mathcal{S}$ is defined on a fixed probability space $(\Omega, \mathcal{F}, \mathbb{P})$. We assume that $X(t)$ has right continuous sample path and Markov semi-group $\mathbf{P}_{t} h(x)=\mathbb{E}_{x}(h(X(t))) \in \mathcal{C}_{b}(\mathcal{S})$ for any $h \in \mathcal{C}_{b}(\mathcal{S})$. Let $\mathcal{D}\left(\mathbf{P}_{t}\right)$ denote the domain of $\mathbf{P}_{t}$ with respect to the supremum norm. That is, the closed vector space $\mathcal{D}\left(\mathbf{P}_{t}\right)=$ $\left\{h \in \mathcal{C}_{b}(\mathcal{S}):\left\|\mathbf{P}_{t} h-h\right\|_{\infty} \rightarrow 0\right.$ as $\left.t \rightarrow 0\right\}$ on which $\mathbf{P}_{t}$ is strongly continuous and such that $\mathbf{P}_{t}: \mathcal{D}\left(\mathbf{P}_{t}\right) \mapsto \mathcal{D}\left(\mathbf{P}_{t}\right)$ and denote by $\mathcal{D}(\mathcal{L})$ the domain of the generator $\mathcal{L}$ : $\mathcal{D}(\mathcal{L}) \mapsto \mathcal{D}\left(\mathbf{P}_{t}\right)$ of the semi-group $\mathbf{P}_{t}$ (i.e. $\mathcal{D}(\mathcal{L}):=\left\{h \in \mathcal{D}\left(\mathbf{P}_{t}\right): t^{-1}\left(\mathbf{P}_{t} h-h\right) \rightarrow \mathcal{L} h\right.$ in $\left(\mathcal{D}\left(\mathbf{P}_{t}\right),\|\cdot\|_{\infty}\right)$ for $\left.\left.t \downarrow 0\right\}\right)$, which is a dense subset of $\left(\mathcal{D}\left(\mathbf{P}_{t}\right),\|\cdot\|_{\infty}\right)$. Recall that if $g \in \mathcal{D}(\mathcal{L})$ then $t \mapsto \mathbf{P}_{t} g: \mathbb{R}_{+} \mapsto \mathcal{D}(\mathcal{L})$ is differentiable and $\partial_{t} \mathbf{P}_{t} g=\mathcal{L} \mathbf{P}_{t} g=\mathbf{P}_{t} \mathcal{L} g$.

For example, when $\mathcal{S}$ is compact one often has $\mathcal{D}\left(\mathbf{P}_{t}\right)=\mathcal{C}_{b}(\mathcal{S})$ whereas for $\mathcal{S}=\mathbb{R}^{d}$ one typically has $\mathcal{D}\left(\mathbf{P}_{t}\right)=\left\{h+c: c \in \mathbb{R}, h \in \mathcal{C}_{0}(\mathcal{S})\right\}$ for the space $\mathcal{C}_{0}(\mathcal{S})$ of continuous functions that vanish at infinity.

The following hypothesis applies throughout.

Assumption 2.1. We assume that $\mathcal{D}\left(\mathbf{P}_{t}\right)$ is an algebra under point-wise multiplication and a dense subset of $L_{2}(\mu)$ for any probability measure $\mu$. We consider test functions in a linear vector space $\mathcal{G} \subseteq \mathcal{D}(\mathcal{L})$ such that $\mathcal{G}$ is an algebra with 
$\mathbf{1} \in \mathcal{G}$ and that $\mathcal{G}$ is dense in $\left(\mathcal{D}\left(\mathbf{P}_{t}\right),\|\cdot\|_{\infty}\right)$. Setting $\widehat{\mathcal{G}}:=\left\{\mathbf{P}_{t} g: g \in \mathcal{G}, t \geq 0\right\}$, we further assume that $\phi(g) \in \mathcal{G}, \mathcal{L} g \in \mathcal{G}$ and $g \widehat{h} \in \mathcal{D}(\mathcal{L})$ for all $g \in \mathcal{G}, \widehat{h} \in \widehat{\mathcal{G}}$ and any $\phi \in \mathcal{C}^{\infty}(\mathbb{R})$.

As usual we say that a finite measure $\mu$ on $\mathcal{S}$ is invariant for $\mathbf{P}_{t}$ if $\left\langle\mathbf{P}_{t} g\right\rangle_{\mu}:=$ $\int \mathbf{P}_{t} g d \mu=\int g d \mu$ for all $g \in B_{\mathcal{S}}$ and $t \geq 0$. Since $\mathcal{G} \subseteq \mathcal{D}(\mathcal{L})$ is dense in $L_{2}(\mu)$, this is equivalent to $\int \mathcal{L} \widehat{g} d \mu=0$ for all $g \in \widehat{\mathcal{G}}$.

A key role is to be played by the symmetric bi-linear operator

$$
\Gamma(f, g)=\mathcal{L}(f g)-f \mathcal{L}(g)-g \mathcal{L}(f),
$$

whose domain is $\mathcal{D}(\boldsymbol{\Gamma}):=\{(f, g): f \in \mathcal{D}(\mathcal{L}), g \in \mathcal{D}(\mathcal{L}), f g \in \mathcal{D}(\mathcal{L})\} \subseteq \mathcal{D}\left(\mathbf{P}_{t}\right) \times$ $\mathcal{D}\left(\mathbf{P}_{t}\right)$. One may instead define $\boldsymbol{\Gamma}$ as the carré du champ operator for the Dirichlet form associated with a $\mu^{0}$-symmetric semi-group $\mathbf{P}_{t}$, thus possibly having a larger domain (c.f. [2, Proposition I.4.1.3]). However, in either case $\mathcal{G} \times \mathcal{G}$ is a dense subset of $\mathcal{D}(\boldsymbol{\Gamma})$, which is all we use in this paper.

Let $\nu=\nu_{0}$ denote the initial measure of $X(0)$ and $\nu_{t}:=\nu \circ \mathbf{P}_{t}$ the corresponding measure of $X(t)$. Fixing $0 \leq s<t<\infty$ and $f \in L_{2}\left(\nu_{s}\right), g \in L_{2}\left(\nu_{t}\right)$, we denote the covariance of the random variables $f(X(s))$ and $g(X(t))$ by $\mathcal{K}_{f, g}(s, t)$. By the Markov property we have,

$$
\mathcal{K}_{f, g}(s, t)=\left\langle\mathbf{P}_{s}\left(f \mathbf{P}_{t-s} g\right)\right\rangle_{\nu}-\left\langle\mathbf{P}_{s} f\right\rangle_{\nu}\left\langle\mathbf{P}_{t} g\right\rangle_{\nu} .
$$

The next lemma provides useful formulas for the derivative of the covariance with respect to $s$.

Lemma 2.2. For any $f, g \in \mathcal{G}$ and all $0 \leq s<t<\infty$,

$$
\begin{aligned}
\partial_{s} \mathcal{K}_{f, g}(s, t) & =\left\langle\mathbf{P}_{s} \mathcal{L}\left(f \mathbf{P}_{t-s} g\right)\right\rangle_{\nu}-\left\langle\mathbf{P}_{s}\left(f \mathbf{P}_{t-s} \mathcal{L} g\right)\right\rangle_{\nu}-\left\langle\mathbf{P}_{s} \mathcal{L} f\right\rangle_{\nu}\left\langle\mathbf{P}_{t} g\right\rangle_{\nu} \\
& =\int_{\mathcal{S}} \mathbf{P}_{s} \boldsymbol{\Gamma}\left(f, \mathbf{P}_{t-s} g\right) d \nu+\mathcal{K}_{\mathcal{L} f, g}(s, t) .
\end{aligned}
$$

Suppose $\nu$ is invariant for $\mathbf{P}_{t}$. Then,

$$
\partial_{s} \mathcal{K}_{f, g}(s, t)=-\left\langle f \mathcal{L} \mathbf{P}_{t-s} g\right\rangle_{\nu} .
$$

If in addition $\mathbf{P}_{t}$ is $\nu$-symmetric (i.e. $\left\langle f \mathbf{P}_{t} g\right\rangle_{\nu}=\left\langle g \mathbf{P}_{t} f\right\rangle_{\nu}$ for all $f, g \in B_{\mathcal{S}}$ ), then

$$
\partial_{s} \mathcal{K}_{f, g}(s, t)=\frac{1}{2}\left\langle\boldsymbol{\Gamma}\left(f, \mathbf{P}_{t-s} g\right)\right\rangle_{\nu} .
$$

Proof: Fix $f, g$ and $s, t$ as in the statement of the lemma, and $\delta>0$. Let $D$ denote the right-hand-side of (2.2) $, h=\mathcal{L} g, \boldsymbol{\Delta}_{\delta}=\mathbf{P}_{\delta}-\mathbf{I}$ and $\widehat{\boldsymbol{\Delta}}_{\delta}=\delta^{-1}\left(\mathbf{P}_{\delta}-\mathbf{I}\right)-\mathcal{L}$. It is not hard to verify that

$$
\begin{aligned}
& \delta^{-1}\left(\mathcal{K}_{f, g}(s+\delta, t)-\mathcal{K}_{f, g}(s, t)\right)-D=\left\langle\mathbf{P}_{s} \widehat{\boldsymbol{\Delta}}_{\delta} f \mathbf{P}_{t-s} g\right\rangle_{\nu}-\left\langle\mathbf{P}_{s+\delta} f \mathbf{P}_{t-s-\delta} \widehat{\boldsymbol{\Delta}}_{\delta} g\right\rangle_{\nu} \\
+ & \left\langle\mathbf{P}_{s+\delta} f \mathbf{P}_{t-s-\delta} \boldsymbol{\Delta}_{\delta} h\right\rangle_{\nu}-\left\langle\mathbf{P}_{s} \boldsymbol{\Delta}_{\delta} f \mathbf{P}_{t-s} h\right\rangle_{\nu}+\left\langle\mathbf{P}_{s} \widehat{\boldsymbol{\Delta}}_{\delta} f\right\rangle_{\nu}\left\langle\mathbf{P}_{t} g\right\rangle_{\nu} .
\end{aligned}
$$

Recall that $\left\{\mathbf{P}_{t}\right\}$ is contractive for the supremum norm while $\left\|\boldsymbol{\Delta}_{\delta} h\right\|_{\infty} \rightarrow 0$ and $\left\|\widehat{\boldsymbol{\Delta}}_{\delta} h\right\|_{\infty} \rightarrow 0$ as $\delta \downarrow 0$ for each fixed $h \in \mathcal{D}(\mathcal{L})$. Since $f$ is bounded with $f, g$, $f \mathbf{P}_{t-s} g$ and $f \mathbf{P}_{t-s} h$ in $\mathcal{D}(\mathcal{L})$, it follows that

$$
\lim _{\delta \downarrow 0} \delta^{-1}\left(\mathcal{K}_{f, g}(s+\delta, t)-\mathcal{K}_{f, g}(s, t)\right)=D .
$$

Similar computation applies for the case of $\delta<0$, thus establishing (2.2). Since $\mathbf{P}_{t-s} \mathcal{L} g=\mathcal{L} \mathbf{P}_{t-s} g$ for $g \in \mathcal{G}$, the equality (2.3) is a direct consequence of (2.1) and 
the definition of $\boldsymbol{\Gamma}(\cdot, \cdot)$. To derive (2.4) note that

$$
D=\left\langle\mathcal{L}\left(f \mathbf{P}_{t-s} g\right)\right\rangle_{\nu_{s}}-\left\langle f \mathcal{L} \mathbf{P}_{t-s} g\right\rangle_{\nu_{s}}-\langle\mathcal{L} f\rangle_{\nu_{s}}\left\langle\mathbf{P}_{t} g\right\rangle_{\nu} .
$$

If $\nu$ is invariant for $\mathbf{P}_{s}$ then $\nu_{s}=\nu$ and further $\langle\mathcal{L} h\rangle_{\nu_{s}}=0$ for any $h \in \mathcal{D}(\mathcal{L})$. Consequently, in this case $D=-\left\langle f \mathcal{L} \mathbf{P}_{t-s} g\right\rangle_{\nu}$ yielding (2.4). If in addition $\mathbf{P}_{t}$ is $\nu$-symmetric, then obviously $\langle f \mathcal{L} h\rangle_{\nu}=\langle h \mathcal{L} f\rangle_{\nu}$ for all $f, h \in \mathcal{D}(\mathcal{L})$, so (2.5) holds by the definition of $\boldsymbol{\Gamma}(\cdot, \cdot)$.

Definition 2.3. The $\mathcal{G}$-convergence as $s \rightarrow \infty$ of probability measures $\nu_{s}$ on $\mathcal{S}$ to a probability measure $\mu$ on $\mathcal{S}$, denoted $\nu_{s} \stackrel{\mathcal{G}}{\rightarrow} \mu$, means that $\langle h\rangle_{\nu_{s}} \rightarrow\langle h\rangle_{\mu}$ as $s \rightarrow \infty$, for any fixed $h \in\{f \widehat{g}, \mathcal{L}(f \widehat{g}): f \in \mathcal{G}, \widehat{g} \in \widehat{\mathcal{G}}\}$.

For example, the weak convergence of $\nu_{s}$ to $\mu$ implies that $\nu_{s} \stackrel{\mathcal{G}}{\rightarrow} \mu$ as well.

Corollary 2.4. If $\nu_{s}=\nu \circ \mathbf{P}_{s} \stackrel{\mathcal{G}}{\rightarrow} \mu$ as $s \rightarrow \infty$, then (2.2) implies that for all $f, g \in \mathcal{G}$, and any fixed $\tau$,

$$
\lim _{s \rightarrow \infty} \partial_{s} \mathcal{K}_{f, g}(s, s+\tau)=\left\langle\mathcal{L}\left(f \mathbf{P}_{\tau} g\right)\right\rangle_{\mu}-\left\langle f \mathbf{P}_{\tau} \mathcal{L} g\right\rangle_{\mu}-\langle\mathcal{L} f\rangle_{\mu}\left\langle\mathbf{P}_{\tau} g\right\rangle_{\mu} .
$$

If in addition $\mathbf{P}_{t}$ is $\mu$-symmetric then by (2.5),

$$
\lim _{s \rightarrow \infty} \partial_{s} \mathcal{K}_{f, g}(s, s+\tau)=\frac{1}{2}\left\langle\boldsymbol{\Gamma}\left(f, \mathbf{P}_{\tau} g\right)\right\rangle_{\mu} .
$$

Given an invariant probability measure $\mu^{0}$ for $\mathbf{P}_{t}$ we consider throughout the following setting (where the Banach space $\mathcal{B}$ is typically $L_{2}\left(\mu^{0}\right)$ or $\left(\mathcal{C}_{b}(\mathcal{S}),\|\cdot\|_{\infty}\right)$ ).

Assumption 2.5. The norm topology on a Banach space $(\mathcal{B},\|\cdot\|)$ of $\mu^{0}$-integrable functions is finer than the one induced by $L_{1}\left(\mu^{0}\right)$ and $\|h\| \leq\|h\|_{\infty}$ for all $h \in$ $\mathcal{D}\left(\mathbf{P}_{t}\right) \subseteq \mathcal{B}$. For each $f \in \mathcal{G}$ there exists a continuous time, homogeneous Markov process $X^{f}(t)$ with a contractive semi-group $\mathbf{P}_{t}^{f}$ on $(\mathcal{B},\|\cdot\|)$ such that $\left\|\mathbf{P}_{t}^{f} h-h\right\| \rightarrow 0$ as $t \rightarrow 0$ for any $h \in \mathcal{D}\left(\mathbf{P}_{t}\right)$ and the finite, positive measure $\mu^{f}$ on $\mathcal{S}$ such that $d \mu^{f} / d \mu^{0}=e^{f}$ is invariant for $\mathbf{P}_{t}^{f}$. Further, $X^{0}(t)=X(t)$, the subspace $\mathcal{G}$ is contained in the domain of the generator $\mathcal{L}^{f}$ of $\mathbf{P}_{t}^{f}$, and $\mathcal{L}^{f} g \in \mathcal{G}$ for all $g \in \mathcal{G}$.

Remark 2.6. It is easy to verify that all results and proofs of this section remain valid in case $\mu^{f}=e^{\Phi(f)} \mu^{0}$ as long as $\Phi: \mathcal{G} \rightarrow B_{\mathcal{S}}$ is such that for any fixed $f \in \mathcal{G}$ the functions $\psi_{\delta}:=\delta^{-1}\left(e^{\Phi(\delta f)}-\delta f-\mathbf{1}\right)$ converge to zero in $L_{1}\left(\mu^{0}\right)$ when $\delta \downarrow 0$.

The FDT is about the relation between derivatives of the covariance at equilibrium and the response of the system to small perturbation out of equilibrium. We turn now to the rigorous definition of the latter (see also Proposition 2.9 for an easy sufficient condition for existence of such response, in case $\mathcal{L}$ is bounded on $(\mathcal{B},\|\cdot\|))$.

Definition 2.7. Assume 2.5 and that for any $f \in \mathcal{G}$ there exists a linear operator $\mathbf{A}_{f}: \mathcal{D}\left(\mathbf{A}_{f}\right) \mapsto \mathcal{B}$ whose domain $\mathcal{D}\left(\mathbf{A}_{f}\right)$ contains $\widehat{\mathcal{G}}$, and such that $s \mapsto \mathbf{A}_{f} \mathbf{P}_{s} g$ is strongly continuous in $(\mathcal{B},\|\cdot\|)$ for each $g \in \mathcal{G}$. If moreover, for any $T \geq 0, \widehat{g} \in \widehat{\mathcal{G}}$, all $t \in[0, T]$ and $\delta>0$,

$$
\left\|\delta^{-1}\left(\mathbf{P}_{t}^{\delta f}-\mathbf{P}_{t}\right) \widehat{g}-\int_{0}^{t} \mathbf{P}_{s} \mathbf{A}_{f} \mathbf{P}_{t-s} \widehat{g} d s\right\| \leq \eta_{\delta} t,
$$


and $\eta_{\delta}=\eta_{\delta}(f, \widehat{g}, T) \rightarrow 0$ as $\delta \downarrow 0$, then we call

$$
R_{X^{f}, g}(s, t)=\mathbf{P}_{s} \mathbf{A}_{f} \mathbf{P}_{t-s} g \in \mathcal{B}
$$

(which for any $t \geq 0$ is strongly integrable on $[0, t]$ ), the response function (at time $t$ and direction $g$ ) for the Markovian perturbations $X^{f}(\cdot)$ on $\mathcal{G}$ (applied at time $s$ ).

Note that $R_{X^{f}, g}(s, t)$ is uniquely defined per given Markovian perturbations $X^{f}(\cdot)$. Indeed, suppose that for some $f \in \mathcal{G}$ the inequality (2.6) holds for the same semi-groups $\mathbf{P}_{t}^{\delta f}$ and both linear operators $\mathbf{A}_{f}$ and $\widetilde{\mathbf{A}}_{f}$. Then, taking $\delta \downarrow 0$ we see that the linear operator $\boldsymbol{\Delta}_{A}=\widetilde{\mathbf{A}}_{f}-\mathbf{A}_{f}$ is such that for all $t>0$ and $\widehat{g} \in \widehat{\mathcal{G}}$,

$$
t^{-1} \int_{0}^{t} \mathbf{P}_{s} \boldsymbol{\Delta}_{A} \widehat{g} d s+t^{-1} \int_{0}^{t} \mathbf{P}_{t-s} \boldsymbol{\Delta}_{A}\left(\mathbf{P}_{s}-\mathbf{I}\right) \widehat{g} d s=\mathbf{0} .
$$

With $\mathbf{P}_{s}$ strongly continuous, upon taking $t \downarrow 0$ the left-most term converges to $\boldsymbol{\Delta}_{A} \widehat{g}$, whereas $t^{-1} \int_{0}^{t} \mathbf{P}_{t-s} \boldsymbol{\Delta}_{A}\left(\mathbf{P}_{s}-\mathbf{I}\right) \widehat{g} d s \rightarrow \mathbf{0}$ by the contractivity of $\mathbf{P}_{t-s}$ on $(\mathcal{B},\|\cdot\|)$ and the assumed strong continuity of $\boldsymbol{\Delta}_{A} \mathbf{P}_{s} \widehat{g}$. Consequently, $\mathbf{A}_{f}=\widetilde{\mathbf{A}}_{f}$ on the set $\widehat{\mathcal{G}}$, and so using either operator in (2.7) leads to the same response function.

As we demonstrate next, $R_{X^{f}, g}(u, a+t+b)$ of (2.7) is merely the effect in "direction" $g$ and at time $a+t+b$, of a small perturbation of the dynamics in "direction" $f$ during the time interval $u \in[a, a+t]$, in agreement with the less formal definition of response function one often finds in the literature.

Corollary 2.8. For any $f, g \in \mathcal{G}$, each $T \geq a, b, t \geq 0$ and all $\delta>0$, a response function of the form 2.7) must satisfy the inequality

$$
\left\|\delta^{-1} \mathbf{P}_{a}\left(\mathbf{P}_{t}^{\delta f}-\mathbf{P}_{t}\right) \mathbf{P}_{b} g-\int_{a}^{a+t} R_{X^{f}, g}(u, a+t+b) d u\right\| \leq \eta_{\delta} t,
$$

for $\eta_{\delta}=\eta_{\delta}(b, f, g, T) \rightarrow 0$ as $\delta \downarrow 0$.

Proof: Using the expression (2.7) to write (2.9) more explicitly, one finds that for $a=0$ the latter is precisely (2.6) for $v=b$, hence it obviously holds. Moreover, in case $a>0$ we merely consider the norm of $\mathbf{P}_{a} h^{\delta}$, where $h^{\delta}$ is the element of $\mathcal{B}$ the norm of which we consider in (2.6). As $\mathbf{P}_{a}$ is contractive on $(\mathcal{B},\|\cdot\|)$, we have that (2.9) holds also in this case.

We provide now an explicit sufficient condition for the existence of a response function of the form (2.7) when $\mathcal{L}^{f}, \mathcal{L}$ and $\mathbf{A}_{f}$ are bounded operators (as is the case in the setting of Section 5 ).

Proposition 2.9. Assume 2.5 holds. If for each $f \in \mathcal{G}$, the operators $\mathbf{A}_{f}, \mathcal{L}, \mathcal{L}^{\delta f}$, $\delta>0$ are bounded on $(\mathcal{B},\|\cdot\|)$ and the corresponding operator norms are such that

$$
\lim _{\delta \downarrow 0}\left\|\delta^{-1}\left(\mathcal{L}^{\delta f}-\mathcal{L}\right)-\mathbf{A}_{f}\right\|=0,
$$

then (2.6) holds and $\mathbf{A}_{f} \mathbf{P}_{s} g$ is strongly continuous, for each $g \in \mathcal{G}$.

Proof: Since $\left\|\mathbf{A}_{f} \mathbf{P}_{s+v} g-\mathbf{A}_{f} \mathbf{P}_{v} g\right\| \leq\left\|\mathbf{A}_{f}\right\|\left\|\mathbf{P}_{s} \widehat{g}-\widehat{g}\right\|$ for $\widehat{g}=\mathbf{P}_{v} g$, the strong continuity of $s \mapsto \mathbf{A}_{f} \mathbf{P}_{s} g$ is a direct consequence of the strong continuity of $\mathbf{P}_{s}$ on its domain. Fixing $f, g \in \mathcal{G}$ and $v \geq 0$ it remains only to show that $t^{-1}\left\|\rho_{t}^{\delta}\right\| \rightarrow 0$ as $\delta \downarrow 0$, uniformly over $t \in(0, T]$, where

$$
\rho_{t}^{\delta}:=\delta^{-1}\left(\mathbf{P}_{t}^{\delta f}-\mathbf{P}_{t}\right) \widehat{g}-\int_{0}^{t} \mathbf{P}_{t-u} \mathbf{A}_{f} \mathbf{P}_{u} \widehat{g} d u .
$$


To this end, let $r_{t}:=-\int_{0}^{t} \mathbf{P}_{t-u} \mathbf{A}_{f} \mathbf{P}_{u} \widehat{g} d u$ and note that

$$
\partial_{t} \rho_{t}^{\delta}=\delta^{-1}\left(\mathcal{L}^{\delta f} \mathbf{P}_{t}^{\delta f}-\mathcal{L} \mathbf{P}_{t}\right) \widehat{g}-\mathbf{A}_{f} \mathbf{P}_{t} \widehat{g}+\mathcal{L} r_{t}
$$

which imply, after some algebraic manipulations, that

$$
\partial_{t} \rho_{t}^{\delta}=\mathcal{L}^{\delta f} \rho_{t}^{\delta}+\zeta_{t}^{\delta}
$$

for

$$
\zeta_{t}^{\delta}:=\left(\mathcal{L}-\mathcal{L}^{\delta f}\right) r_{t}+\left[\delta^{-1}\left(\mathcal{L}^{\delta f}-\mathcal{L}\right)-\mathbf{A}_{f}\right] \mathbf{P}_{t} \widehat{g} .
$$

It is not hard to show that the solution $\rho_{t}^{\delta}$ of (2.11) with initial condition $\rho_{0}^{\delta}=\mathbf{0}$, is

$$
\rho_{t}^{\delta}=\int_{0}^{t} \mathbf{P}_{t-s}^{\delta f} \zeta_{s}^{\delta} d s
$$

With both $\mathbf{P}_{t}^{\delta f}$ and $\mathbf{P}_{t}$ contractive on $(\mathcal{B},\|\cdot\|)$, we thus have that $\left\|r_{t}\right\| \leq t\left\|\mathbf{A}_{f}\right\|\|g\|$ and for any $t \in[0, T]$,

$\left\|\rho_{t}^{\delta}\right\| \leq \int_{0}^{t}\left\|\zeta_{s}^{\delta}\right\| d s \leq t\left[T\left\|\mathcal{L}-\mathcal{L}^{\delta f}\right\|\left\|\mathbf{A}_{f}\right\|+\left\|\delta^{-1}\left(\mathcal{L}^{\delta f}-\mathcal{L}\right)-\mathbf{A}_{f}\right\|\right]\|g\|=: \eta_{\delta}(f, g, T) t$.

Finally, note that from (2.10) we have that $\eta_{\delta} \rightarrow 0$ as $\delta \downarrow 0$.

Our next theorem characterizes the type of response functions one may find. It also proves the FDT, showing that if $X^{f}(\cdot)$ has a response function $R_{X^{f}, g}(s, t)$, then the average of the response function according to an initial measure $\nu_{0}=\mu^{0}$ which is invariant for $X(\cdot)$, equals the time derivative of the covariance of $X(\cdot)$ under the same initial measure.

Theorem 2.10 (Fluctuation Dissipation Theorem). Let $f \in \mathcal{G}$. If $X^{f}(\cdot)$ has a response function, then $\mathbf{A}_{f} \mathbf{1}=\mathbf{0}$ and $\mathbf{A}_{r f}=r \mathbf{A}_{f}$ for all $r>0$. Further, then $\left\langle\left(\mathbf{A}_{f}+f \mathcal{L}\right) \widehat{g}\right\rangle_{\mu^{0}}=0$ for all $\widehat{g} \in \widehat{\mathcal{G}}$ and consequently, if the initial measure $\nu_{0}=\mu^{0}$, then for any $s<t$,

$$
\partial_{s} \mathcal{K}_{f, g}(s, t)=\left\langle R_{X^{f}, g}(s, t)\right\rangle_{\nu_{0}} .
$$

Remark 2.11. If $\mathbf{P}_{t}$ is $\mu^{0}$-symmetric and $f, g \in L_{2}\left(\mu^{0}\right)$ with $(f, g) \in \mathcal{D}(\boldsymbol{\Gamma})$, then by spectral decomposition we have the Green-Kubo formula

$$
-\langle f \mathcal{L} g\rangle_{\mu^{0}}=\frac{1}{2}\langle\boldsymbol{\Gamma}(f, g)\rangle_{\mu^{0}}=\int_{0}^{\infty}\left[\left\langle\left(\mathbf{P}_{s} \mathcal{L} f\right)(\mathcal{L} g)\right\rangle_{\mu^{0}}\right] d s
$$

(c.f. [15, Theorem 4.3.8]). Applying it for $f$ and $\mathbf{P}_{t-s} g$, we get the alternative expression

$$
\partial_{s} \mathcal{K}_{f, g}(s, t)=\int_{0}^{\infty}\left[\left\langle\left(\mathbf{P}_{s} \mathcal{L} f\right)\left(\mathcal{L} \mathbf{P}_{t-s} g\right)\right\rangle_{\mu^{0}}\right] d s
$$

for the dissipation term. In contrast with (2.13), this identity does not involve a perturbation of the given Markovian dynamic.

Proof: Fix $f \in \mathcal{G}$. If $g=\mathbf{1}$ then $\mathbf{P}_{u} g=\mathbf{P}_{u}^{\delta f} g=\mathbf{1}$ for all $\delta>0$ and $u \geq 0$, so in this case taking $\delta \downarrow 0$ in (2.6) we find that for any $t>0$,

$$
t^{-1} \int_{0}^{t} \mathbf{P}_{s} \mathbf{A}_{f} \mathbf{1} d s=\mathbf{0} \text {. }
$$

Thus, taking $t \downarrow 0$ we have that $\mathbf{A}_{f} \mathbf{1}=\mathbf{0}$. 
Next, fixing $r>0$, note that $\mathbf{P}_{t}^{\delta(r f)}=\mathbf{P}_{t}^{(\delta r) f}$ for all $\delta>0$, hence by (2.6) we have for $\boldsymbol{\Delta}_{A}=\mathbf{A}_{r f}-r \mathbf{A}_{f}$,

$$
\left\|\int_{0}^{t} \mathbf{P}_{s} \boldsymbol{\Delta}_{A} \mathbf{P}_{t-s} \widehat{g} d s\right\| \leq\left[\eta_{\delta}(r f, \widehat{g}, T)+r \eta_{\delta r}(f, \widehat{g}, T)\right] t .
$$

Taking $\delta \downarrow 0$ this implies that (2.8) holds for $\boldsymbol{\Delta}_{A}=\mathbf{A}_{r f}-r \mathbf{A}_{f}$ and all $t>0$. Hence, by the argument we provided immediately following (2.8), we deduce that $\boldsymbol{\Delta}_{A} \widehat{g}=\mathbf{0}$ for all $\widehat{g} \in \widehat{\mathcal{G}}$. That is, without loss of generality we may assume that $\mathbf{A}_{r f}=r \mathbf{A}_{f}$, as claimed.

Let $\psi_{\delta}=\delta^{-1}\left(e^{\delta f}-\delta f-\mathbf{1}\right)$. Since $\mu^{\delta f}$ is invariant for $\mathbf{P}_{t}^{\delta f}$ and $\mu^{0}$ is invariant for $\mathbf{P}_{t}$, it follows that

$$
\begin{aligned}
\left\langle\delta^{-1}\left(\mathbf{P}_{t}^{\delta f}-\mathbf{P}_{t}\right) \widehat{g}\right\rangle_{\mu^{0}} & =\left\langle\psi_{\delta}\left(\mathbf{I}-\mathbf{P}_{t}^{\delta f}\right) \widehat{g}\right\rangle_{\mu^{0}}-\left\langle f\left(\mathbf{P}_{t}^{\delta f}-\mathbf{P}_{t}\right) \widehat{g}\right\rangle_{\mu^{0}}-\left\langle f\left(\mathbf{P}_{t}-\mathbf{I}\right) \widehat{g}\right\rangle_{\mu^{0}} \\
& :=F_{1}(\delta)+F_{2}(\delta)+F_{3} .
\end{aligned}
$$

With (2.6) implying that $\left\|\left(\mathbf{P}_{t}^{\delta f}-\mathbf{P}_{t}\right) \widehat{g}\right\| \rightarrow 0$ as $\delta \downarrow 0$ (hence so does \langle|$\left(\mathbf{P}_{t}^{\delta f}-\right.$ $\left.\left.\mathbf{P}_{t}\right) \widehat{g}|\rangle_{\mu^{0}}\right)$, and $\|f\|_{\infty}<\infty$ we deduce that $F_{2}(\delta) \rightarrow 0$. Further, $\left\langle\left|\psi_{\delta}\right|\right\rangle_{\mu^{0}} \rightarrow 0$ when $\delta \downarrow 0$ (since $f$ is bounded), while $\left\|\left(\mathbf{I}-\mathbf{P}_{t}^{\delta f}\right) \widehat{g}\right\|_{\infty} \leq 2\|\widehat{g}\|_{\infty}$ is uniformly bounded in $\delta$, resulting with $F_{1}(\delta) \rightarrow 0$ as well. We thus deduce by considering the limit $\delta \downarrow 0$ in (2.6), and applying Fubini's theorem, that for any $t>0$ and $\widehat{g} \in \widehat{\mathcal{G}}$,

$$
\left\langle f t^{-1}\left(\mathbf{P}_{t}-\mathbf{I}\right) \widehat{g}\right\rangle_{\mu^{0}}+t^{-1} \int_{0}^{t}\left\langle\mathbf{P}_{t-s} \mathbf{A}_{f} \mathbf{P}_{s} \widehat{g}\right\rangle_{\mu^{0}} d s=0 .
$$

Further, with $\mu^{0}$ invariant for $\mathbf{P}_{t}$ and having assumed strong continuity of $s \mapsto$ $\mathbf{A}_{f} \mathbf{P}_{s} \widehat{g}$, upon taking $t \downarrow 0$ we find that

$$
\langle f \mathcal{L} \widehat{g}\rangle_{\mu^{0}}+\left\langle\mathbf{A}_{f} \widehat{g}\right\rangle_{\mu^{0}}=0
$$

as claimed.

Next, recall (2.4) and (2.7) that for $\nu_{0}=\mu^{0}$ which is invariant for $\mathbf{P}_{s}$ and for any finite $s<t$,

$$
\partial_{s} \mathcal{K}_{f, g}(s, t)-\left\langle R_{X^{f}, g}(s, t)\right\rangle_{\mu^{0}}=-\left\langle f \mathcal{L} \mathbf{P}_{t-s} g\right\rangle_{\mu^{0}}-\left\langle\mathbf{A}_{f} \mathbf{P}_{t-s} g\right\rangle_{\mu^{0}}=0,
$$

where the right-most identity is precisely (2.14).

Combining Corollary 2.4 and Theorem 2.10 we deduce the existence of the FDT regime in out-of-equilibrium dynamics whenever $X(s)$ converges in law (in the appropriate sense), with the limiting measure $\mu^{0}$ being invariant for $X(\cdot)$. That is, the FDT relation (2.13) then asymptotically holds for $t-s=\tau$ fixed, in the limit $s \rightarrow \infty$. Specifically, similar to Definition 2.3 we define the notion of $\mathcal{G}_{f}$-convergence as follows.

Definition 2.12. The $\mathcal{G}_{f}$-convergence of probability measures $\nu_{s}$ on $\mathcal{S}$ to a probability measure $\mu$ on $\mathcal{S}$, denoted $\nu_{s} \stackrel{\mathcal{G}_{f}}{\rightarrow} \mu$, means that $\langle h\rangle_{\nu_{s}} \rightarrow\langle h\rangle_{\mu}$ as $s \rightarrow \infty$, for any fixed $h \in\left\{f \widehat{g}, \mathcal{L}(f \widehat{g}), \mathbf{A}_{f} \widehat{g}: \widehat{g} \in \widehat{\mathcal{G}}\right\}$ (implicitly assuming that $\mathbf{A}_{f} \widehat{g} \in L_{1}\left(\nu_{s}\right)$ for all s large enough).

Corollary 2.13. Let $\nu_{0}$ denote the initial measure of $X(0)$ for an $\mathcal{S}$-valued, continuous time, homogeneous, strong Markov process $X(t)$ such that $X^{f}(\cdot)$ has a response 
function (in the sense of Definition 2.7). Suppose further that $\nu_{s}=\nu_{0} \circ \mathbf{P}_{s} \stackrel{\mathcal{G}_{f}}{\rightarrow} \mu^{0}$ as $s \rightarrow \infty$. Then, for any $g \in \mathcal{G}$ and fixed $\tau \geq 0$,

$$
\lim _{s \rightarrow \infty} \partial_{s} \mathcal{K}_{f, g}(s, s+\tau)=-\left\langle f \mathcal{L} \mathbf{P}_{\tau} g\right\rangle_{\mu^{0}}=\lim _{s \rightarrow \infty}\left\langle R_{X^{f}, g}(s, s+\tau)\right\rangle_{\nu_{0}} .
$$

Proof: The left side of the identity follows from the formula for the limit of $\partial_{s} \mathcal{K}_{f, g}(s, s+\tau)$ given in Corollary 2.4 and the fact that $\mu^{0}$ is invariant for $\mathbf{P}_{t}$ so $\langle\mathcal{L} h\rangle_{\mu^{0}}=0$ for all $h \in \mathcal{D}(\mathcal{L})$. Recall (2.7) that $\left\langle R_{X^{f}, g}(s, s+\tau)\right\rangle_{\nu_{0}}=\left\langle\mathbf{A}_{f} \mathbf{P}_{\tau} g\right\rangle_{\nu_{s}}$ which converges to $\left\langle\mathbf{A}_{f} \mathbf{P}_{\tau} g\right\rangle_{\mu^{0}}$ as $s \rightarrow \infty$. From Theorem 2.10 we have that $\left\langle\left(\mathbf{A}_{f}+f \mathcal{L}\right) \mathbf{P}_{\tau} g\right\rangle_{\mu^{0}}=0$, which thus yields the right side of the stated identity.

We conclude this section with a statement characterizing response functions for symmetric perturbations.

Proposition 2.14. Assume 2.5 holds for the Hilbert space $\mathcal{B}=L_{2}\left(\mu^{0}\right)$ and that for each $f \in \mathcal{G}$ the semi-group $\mathbf{P}_{t}^{f}$ of $X^{f}(t)$ is $\mu^{f}$-symmetric. Then, any response function of the form (2.7) is based on $\mathbf{A}_{f}=\mathbf{B}_{f}-f \mathcal{L}$ for a linear operator $\mathbf{B}_{f}$ which is $\mu^{0}$-symmetric on $\widehat{\mathcal{G}}$.

Remark 2.15. The stated $\mu^{0}$-symmetry of $\mathbf{B}_{f}=\mathbf{A}_{f}+f \mathcal{L}$ is necessary in this setting of symmetric perturbations. However, typically more is required from $\mathbf{B}_{f}$ in order to assure the existence of a response function of the form 2.7).

Proof: Note that for any $\delta>0$ and all $\widehat{g}, \widehat{h} \in \widehat{\mathcal{G}}$,

$$
\begin{aligned}
F_{\delta}(\widehat{h}, \widehat{g}):= & \delta^{-1}\left\langle\widehat{h}\left(\mathbf{P}_{t}^{\delta f}-\mathbf{P}_{t}\right) \widehat{g}\right\rangle_{\mu^{0}}+\left\langle\widehat{h} f\left(\mathbf{P}_{t}-\mathbf{I}\right) \widehat{g}\right\rangle_{\mu^{0}} \\
= & \left\langle\widehat{h} \psi_{\delta}\left(\mathbf{I}-\mathbf{P}_{t}^{\delta f}\right) \widehat{g}\right\rangle_{\mu^{0}}-\left\langle\widehat{h} f\left(\mathbf{P}_{t}^{\delta f}-\mathbf{P}_{t}\right) \widehat{g}\right\rangle_{\mu^{0}} \\
+ & \delta^{-1}\left\langle\widehat{h}\left(\mathbf{P}_{t}^{\delta f}-\mathbf{I}\right) \widehat{g}\right\rangle_{\mu^{\delta f}}-\delta^{-1}\left\langle\widehat{h}\left(\mathbf{P}_{t}-\mathbf{I}\right) \widehat{g}\right\rangle_{\mu^{0}} \\
=: & F_{1}(\delta, \widehat{h}, \widehat{g})+F_{2}(\delta, \widehat{h}, \widehat{g})+F_{3}(\delta, \widehat{h}, \widehat{g})+F_{4}(\delta, \widehat{h}, \widehat{g}),
\end{aligned}
$$

where $\psi_{\delta}=\delta^{-1}\left(e^{\delta f}-\delta f-\mathbf{1}\right)$. Recall that $\left\|\left(\mathbf{I}-\mathbf{P}_{t}^{\delta f}\right) \widehat{g}\right\|_{\infty} \leq 2\|\widehat{g}\|_{\infty}, \widehat{h}$ is bounded and $\left\langle\left|\psi_{\delta}\right|\right\rangle_{\mu^{0}} \rightarrow 0$, hence $F_{1}(\delta, \widehat{h}, \widehat{g}) \rightarrow 0$ as $\delta \downarrow 0$. Further, $\left\langle\left|\left(\mathbf{P}_{t}^{\delta f}-\mathbf{P}_{t}\right) \widehat{g}\right|\right\rangle_{\mu^{0}} \rightarrow 0$ hence also $F_{2}(\delta, \widehat{h}, \widehat{g}) \rightarrow 0$ as $\delta \downarrow 0$. By the $\mu^{\delta f}$-symmetry of $\mathbf{P}_{t}^{\delta f}$ and the $\mu^{0}$-symmetry of $\mathbf{P}_{t}$, it follows that

$$
F_{3}(\delta, \widehat{h}, \widehat{g})=F_{3}(\delta, \widehat{g}, \widehat{h}), \quad F_{4}(\delta, \widehat{h}, \widehat{g})=F_{4}(\delta, \widehat{g}, \widehat{h}),
$$

for any $\delta>0$. Consequently, as $\delta \downarrow 0$,

$$
F_{\delta}(\widehat{h}, \widehat{g})-F_{\delta}(\widehat{g}, \widehat{h}) \rightarrow 0
$$

which by (2.6) and the $\mu^{0}$-symmetry of $\mathbf{P}_{t-u}$ amounts to

$$
E_{t}(\widehat{h}, \widehat{g})=E_{t}(\widehat{g}, \widehat{h}) \text {, }
$$

where

$$
E_{t}(\widehat{h}, \widehat{g}):=\int_{0}^{t}\left\langle\left(\mathbf{P}_{t-s} \widehat{h}\right)\left(\mathbf{A}_{f} \mathbf{P}_{s} \widehat{g}\right)\right\rangle_{\mu^{0}} d s+\left\langle\widehat{h} f\left(\mathbf{P}_{t}-\mathbf{I}\right) \widehat{g}\right\rangle_{\mu^{0}} .
$$

Since $s \mapsto \mathbf{A}_{f} \mathbf{P}_{s} \widehat{g}$ is strongly continuous (as part of Definition 2.7 of the response function), it follows that as $t \downarrow 0$,

$t^{-1} \int_{0}^{t}\left\langle\widehat{h} \mathbf{A}_{f} \mathbf{P}_{s} \widehat{g}\right\rangle_{\mu^{0}} d s+t^{-1}\left\langle\widehat{h} f\left(\mathbf{P}_{t}-\mathbf{I}\right) \widehat{g}\right\rangle_{\mu^{0}} \rightarrow\left\langle\widehat{h} \mathbf{A}_{f} \widehat{g}\right\rangle_{\mu^{0}}+\langle\widehat{h} f \mathcal{L} \widehat{g}\rangle_{\mu^{0}}=\left\langle\widehat{h} \mathbf{B}_{f} \widehat{g}\right\rangle_{\mu^{0}}$ 
Further, for all $u>0$,

$$
\left(\mathbf{P}_{u}-\mathbf{I}\right) \widehat{h}=\int_{0}^{u} \mathbf{P}_{v} \mathcal{L} \widehat{h} d v,
$$

hence $\left\|\left(\mathbf{P}_{u}-\mathbf{I}\right) \widehat{h}\right\|_{\infty} \leq u\|\mathcal{L} \widehat{h}\|_{\infty}$, while the strong continuity of $\mathbf{A}_{f} \mathbf{P}_{s} \widehat{g}$ implies that $\sup _{s \leq t}\left\|\mathbf{A}_{f} \mathbf{P}_{s} \widehat{g}\right\| \rightarrow\left\|\mathbf{A}_{f} \widehat{g}\right\|<\infty$ as $t \downarrow 0$. Taken together, these imply that

$$
\lim _{t \downarrow 0} t^{-1} \int_{0}^{t}\left\langle\left(\left(\mathbf{P}_{t-s}-\mathbf{I}\right) \widehat{h}\right)\left(\mathbf{A}_{f} \mathbf{P}_{s} \widehat{g}\right)\right\rangle_{\mu^{0}} d s=0
$$

and consequently we have also that

$$
t^{-1} E_{t}(\widehat{h}, \widehat{g}) \rightarrow\left\langle\widehat{h} \mathbf{B}_{f} \widehat{g}\right\rangle_{\mu^{0}} .
$$

We thus conclude, based on (2.16), that $\left\langle\widehat{h} \mathbf{B}_{f} \widehat{g}\right\rangle_{\mu^{0}}=\left\langle\widehat{g} \mathbf{B}_{f} \widehat{h}\right\rangle_{\mu^{0}}$ for any $\widehat{g}, \widehat{h} \in \widehat{\mathcal{G}}$, as claimed.

\section{Generic Markov perturbations}

In this section we construct several generic Markov perturbations for which Assumption 2.5 holds. Our presentation is somewhat technical because we aim at addressing a rather general framework. The reader may thus benefit from considering first the concrete examples of Sections 5 and 6

Time change. Our first construction corresponds to changing the clock as follows. For each fixed $f \in \mathcal{G}$ and $s \geq 0$ let

$$
t^{f}(s, \omega):=\int_{0}^{s} e^{f(X(u))} d u .
$$

Note that $t^{f}: \mathbb{R}_{+} \times \Omega \rightarrow \mathbb{R}_{+}$is a measurable stochastic process the sample path of which are everywhere differentiable with $\frac{d}{d s} t^{f}=e^{f(X(s))}$ bounded and bounded away from zero. Its inverse, $\tau^{f}(t):=\inf \left\{s \geq 0: t^{f}(s) \geq t\right\}$ is thus also a measurable stochastic process, the sample path of which are everywhere differentiable with $\frac{d}{d t} \tau^{f}=e^{-f\left(X\left(\tau^{f}(t)\right)\right)}$ uniformly (in $t$ and $\omega$ ) bounded and bounded away from zero.

Proposition 3.1. Assumption 2.5 holds for $(\mathcal{B},\|\cdot\|)=\left(\mathcal{C}_{b}(\mathcal{S}),\|\cdot\|_{\infty}\right)$ and the Markov process $X_{0}^{f}(t)=X\left(\tau^{f}(t)\right)$. Further, the generator $\mathcal{L}_{0}^{f}$ of the semi-group $\left(\mathbf{P}_{0}^{f}\right)_{t}$ of $X_{0}^{f}(t)$ is such that $\mathcal{D}(\mathcal{L})=\mathcal{D}\left(\mathcal{L}_{0}^{f}\right)$ and $\mathcal{L}_{0}^{f} g=e^{-f} \mathcal{L} g$ for any $g \in \mathcal{D}(\mathcal{L})$.

Proof: Obviously, $\left\{\tau^{f}(t) \leq s\right\}=\left\{t^{f}(s) \geq t\right\}$ is in $\mathcal{F}_{s}=\sigma(X(u): 0 \leq u \leq s)$ by the right continuity and boundedness of $u \mapsto \exp (f(X(u)))$. Hence, for each fixed $t$, the random variable $\tau^{f}(t)$ is a stopping time with respect to the canonical filtration $\mathcal{F}_{s}$ of $\{X(\cdot)\}$. Similarly, the stochastic process

$$
\tau^{f}(t)=\int_{0}^{t} e^{-f\left(X_{0}^{f}(v)\right)} d v
$$

is adapted to the canonical filtration $\mathcal{F}_{t}^{f}=\sigma\left(X_{0}^{f}(u): 0 \leq u \leq t\right)$ of $\left\{X_{0}^{f}(\cdot)\right\}$. The strict monotonicity and continuity of $t \mapsto \tau^{f}(t)$ imply that $\mathcal{F}_{t}^{f}=\mathcal{F}_{\tau^{f}(t)}$ and further it is not hard to check that $\tau^{f}(t, \omega)$ have the regeneration property

$$
\tau^{f}(t, \omega)=\tau^{f}(s, \omega)+\tau^{f}\left(t-s, \theta^{\tau^{f}(s, \omega)} \omega\right),
$$


for any $t>s>0$ (where $\theta^{u} \omega(\cdot)=\omega(u+\cdot)$ denotes the usual shift operator). Thus, for any $h \in B_{\mathcal{S}}, t>s>0$ and $x \in \mathcal{S}$, by the strong Markov property of $X(\cdot)$ at $\tau^{f}(s)$

$$
\begin{aligned}
\mathbb{E}_{x}\left[h\left(X_{0}^{f}(t)\right) \mid \mathcal{F}_{s}^{f}\right] & =\mathbb{E}_{x}\left[h\left(X\left(\tau^{f}(t)\right)\right) \mid \mathcal{F}_{\tau^{f}(s)}\right] \\
& =\mathbb{E}_{X\left(\tau^{f}(s)\right)}\left[h\left(\tilde{X}\left(\tau^{f}(t-s)\right)\right)\right]=\mathbb{E}_{X_{0}^{f}(s)}\left[h\left(\tilde{X}_{0}^{f}(t-s)\right)\right],
\end{aligned}
$$

where $\tilde{X}(\cdot)$ and $\tilde{X}_{0}^{f}(\cdot)$ denote independent copies of $X(\cdot)$ and $X_{0}^{f}(\cdot)$, respectively. The Markov property of $X_{0}^{f}(\cdot)$ then follows from (3.1) by standard arguments. Since $X_{0}^{f}(\cdot)$ assumes its values in a complete, separable metric space, the contractive semigroup $\mathbf{P}_{t}^{f} h=\mathbb{E}_{x}\left(h\left(X_{0}^{f}(t)\right)\right)$ is well defined on $\left(\mathcal{C}_{b}(\mathcal{S}),\|\cdot\|_{\infty}\right)$. Further, by the change of variable $v=\tau^{f}(u)$ its resolvent is given by

$$
\mathbf{R}_{\lambda}^{f} h=\mathbb{E}_{x}\left[\int_{0}^{\infty} e^{-\lambda u} h\left(X_{0}^{f}(u)\right) d u\right]=\mathbb{E}_{x}\left[\int_{0}^{\infty} e^{-\lambda t^{f}(v)} e^{f(X(v))} h(X(v)) d v\right] .
$$

Note that $\lambda t^{f}(s)=\lambda c s-\int_{0}^{s} \xi(X(u)) d u$ for positive, finite $c=\exp \left(\|f\|_{\infty}\right)$ and the continuous function $\xi(x)=\lambda\left(c-e^{f(x)}\right) \geq 0$ with $\|\xi\|_{\infty}<\lambda c$. The linear operator $\mathbf{R}_{\lambda c} \xi: \mathcal{D}\left(\mathbf{P}_{t}\right) \mapsto \mathcal{D}(\mathcal{L})$ such that

$$
\left(\mathbf{R}_{\lambda c} \xi\right) g=\mathbb{E}_{x}\left[\int_{0}^{\infty} e^{-\lambda c s} \xi(X(s)) g(X(s)) d s\right]
$$

is thus strictly contractive, and since the series

$$
\mathbf{R}_{\lambda}^{f} h=\sum_{k \geq 0}\left(\mathbf{R}_{\lambda c} \xi\right)^{k} \mathbf{R}_{\lambda c}\left(e^{f} h\right)
$$

converges uniformly, it follows that $\mathbf{R}_{\lambda}^{f}: \mathcal{D}\left(\mathbf{P}_{t}\right) \mapsto \mathcal{D}\left(\mathbf{P}_{t}\right)$ and consequently $\mathbf{P}_{t}^{f}$ : $\mathcal{C}_{b}(\mathcal{S}) \mapsto \mathcal{C}_{b}(\mathcal{S})$

Fixing $g \in \mathcal{D}(\mathcal{L})$ recall that $g(X(\cdot))$ and $(\mathcal{L} g)(X(\cdot))$ are bounded right-continuous functions, with $M(t):=g(X(t))-g(X(0))-\int_{0}^{t}(\mathcal{L} g)(X(v)) d v$ a right-continuous martingale with respect to the filtration $\mathcal{F}_{t}$. With $M(0)=0$ and $\tau^{f}(t)$ bounded above uniformly in $\omega$, by Doob's optional sampling theorem $\mathbb{E}_{x} M\left(\tau^{f}(t)\right)=0$ for any $x \in \mathcal{S}$. By the change of variable $v=\tau^{f}(u)$ and Fubini, this amounts to

$$
\mathbf{P}_{t}^{f} g(x)=g(x)+\int_{0}^{t} \mathbb{E}_{x}\left[\left(e^{-f} \mathcal{L} g\right)\left(X^{f}(u)\right)\right] d u=g(x)+\int_{0}^{t}\left(\mathbf{P}_{u}^{f} e^{-f} \mathcal{L} g\right)(x) d u
$$

Let $\widetilde{g}=e^{-f} \mathcal{L} g \in \mathcal{D}\left(\mathbf{P}_{t}\right)$. Since $\mathbf{P}_{u}^{f}$ is contractive on $\mathcal{D}\left(\mathbf{P}_{t}\right)$, it follows from (3.2) that $\left\|\mathbf{P}_{t}^{f} g-g\right\|_{\infty} \leq t\|\widetilde{g}\|_{\infty}$. In particular, $\left\|\mathbf{P}_{t}^{f} g-g\right\|_{\infty} \rightarrow 0$ as $t \downarrow 0$, for all $g \in \mathcal{G}$. With $\mathcal{G}$ dense in the closed vector space $\left(\mathcal{D}\left(\mathbf{P}_{t}\right),\|\cdot\|_{\infty}\right)$, we have that $\mathbf{P}_{t}^{f}$ is strongly continuous there. The uniformly bounded and strongly continuous $\mathbf{P}_{u}^{f} \widetilde{g}:[0, t] \mapsto$ $\mathcal{C}_{b}(\mathcal{S})$ is also strongly integrable, so 3.2 implies that for any $g \in \mathcal{D}(\mathcal{L})$,

$$
\lim _{t \downarrow 0}\left\|t^{-1}\left(\mathbf{P}_{t}^{f} g-g\right)-\widetilde{g}\right\|_{\infty}=\lim _{t \downarrow 0}\left\|t^{-1} \int_{0}^{t} \mathbf{P}_{u}^{f} \widetilde{g} d u-\widetilde{g}\right\|_{\infty}=0
$$

We have thus seen that $\mathcal{D}(\mathcal{L}) \subseteq \mathcal{D}\left(\mathcal{L}_{0}^{f}\right)$ with $\mathcal{L}_{0}^{f} g=e^{-f} \mathcal{L} g$ for all $g \in \mathcal{D}(\mathcal{L})$.

We next prove that if $g \in \mathcal{D}\left(\mathcal{L}_{0}^{f}\right)$, then necessarily $g \in \mathcal{D}(\mathcal{L})$. To this end, observe that the sample path of $X^{f}(t)$ inherits the right continuity of those of $X(t)$, and fixing $g \in \mathcal{D}\left(\mathcal{L}_{0}^{f}\right)$ (which is thus continuous and bounded), we have the right-continuous martingale $M^{f}(s)=g\left(X^{f}(s)\right)-g\left(X^{f}(0)\right)-\int_{0}^{s}\left(\mathcal{L}_{0}^{f} g\right)\left(X^{f}(v)\right) d v$ 
with respect to the filtration $\mathcal{F}_{s}^{f}$. With $M^{f}(0)=0$ and the stopping time $t^{f}(s)$ for the latter filtration bounded above uniformly (in $\omega$ ), by the optional sampling theorem $\mathbb{E}_{x} M^{f}\left(t^{f}(s)\right)=0$ for all $x \in \mathcal{S}$. Since $X^{f}\left(t^{f}(u)\right)=X(u)$, using Fubini and the change of variable $v=t^{f}(u)$ we get in analogy to (3.2) that

$$
\mathbf{P}_{s} g(x)=g(x)+\int_{0}^{s}\left(\mathbf{P}_{u} e^{f} \mathcal{L}_{0}^{f} g\right)(x) d u,
$$

which by the uniform boundedness and strong continuity of $\mathbf{P}_{u} e^{f} \mathcal{L}_{0}^{f} g$ results with $g \in \mathcal{D}(\mathcal{L})$ (and $\mathcal{L} g=e^{f} \mathcal{L}_{0}^{f} g$ ), completing the proof that $\mathcal{L}_{0}^{f}=e^{-f} \mathcal{L}$.

In particular, $\mathcal{G} \subseteq \mathcal{D}\left(\mathcal{L}_{0}^{f}\right)$ and since $\mathcal{G}$ is an algebra containing both $e^{-f}$ and $\mathcal{L} g$, it follows that $\mathcal{L}_{0}^{f} g \in \mathcal{G}$ for any $f, g \in \mathcal{G}$. It remains just to verify that $\mu^{f}$ is invariant for $\mathbf{P}_{t}^{f}$, that is, $\int \mathcal{L}_{0}^{f} \mathbf{P}_{t}^{f} g d \mu^{f}=0$ for all $t>0$ and $g \in \mathcal{G}$. We have already shown that $\int \mathcal{L}_{0}^{f} \mathbf{P}_{t}^{f} g d \mu^{f}=\int \mathcal{L}\left(\mathbf{P}_{t}^{f} g\right) d \mu^{0}$. The latter is zero since $\mathbf{P}_{t}^{f} g \in \mathcal{D}\left(\mathcal{L}_{0}^{f}\right)=\mathcal{D}(\mathcal{L})$ and $\mu^{0}$ is invariant for $\mathbf{P}_{t}$, so the proof of the proposition is complete.

Perturbations for symmetric processes. Relying on the powerful technology of Dirichlet forms (c.f. [2]), the second generic case we consider is that of a generalized Langevin dynamics for a $\mu^{0}$-symmetric process.

Proposition 3.2. Suppose $\mathbf{P}_{t}$ is $\mu^{0}$-symmetric. Then, for any $f \in \mathcal{G}$ there exists a Markov process $X_{1}^{f}$ such that the generator of its $\mu^{f}$-symmetric, strongly continuous semi-group $\left(\overline{\mathbf{P}}_{1}^{f}\right)_{t}$ on the Hilbert space $\mathcal{B}=L_{2}\left(\mu^{f}\right)$, satisfies

$$
\overline{\mathcal{L}}_{1}^{f} g=\mathcal{L} g+\frac{1}{2} e^{-f} \boldsymbol{\Gamma}\left(e^{f}, g\right), \quad \forall g \in \mathcal{G}
$$

and for which Assumption 2.5 holds.

The Leibniz rule $\boldsymbol{\Gamma}(f h, g)=h \boldsymbol{\Gamma}(f, g)+f \boldsymbol{\Gamma}(h, g)$ applies whenever $\mathcal{L}$ is the generator of a Markov process of continuous trajectories $t \mapsto X(t)$ (c.f. [1]), resulting with $\overline{\mathcal{L}}_{1}^{f} g=\mathcal{L} g+\frac{1}{2} \boldsymbol{\Gamma}(f, g)$ as soon as $\boldsymbol{\Gamma}\left(\sum_{k>n} \frac{f^{k}}{k !}, g\right) \rightarrow 0$ when $n \rightarrow \infty$. We call $X_{1}^{f}$ a generalized Langevin dynamics since for $\mathcal{S}$ a finite dimensional, compact, connected smooth manifold without boundary, the perturbed process $X_{1}^{f}(\cdot)$ is then obtained by adding to the original (diffusion) process a drift of a gradient form (c.f. Section 6).

Remark 3.3. Proposition 3.2 provides us with a semi-group $\left(\overline{\mathbf{P}}_{1}^{f}\right)_{t}$ of $X_{1}^{f}$ that is defined only for $\mu^{0}$ almost every $x \in \mathcal{S}$. In most interesting specific situations one easily shows that $\left(\overline{\mathbf{P}}_{1}^{f}\right)_{t}$ is the unique extension to $L_{2}\left(\mu^{f}\right)$ of a semi-group $\left(\mathbf{P}_{1}^{f}\right)_{t}$ that is strongly continuous on $\left(\mathcal{D}\left(\mathbf{P}_{t}\right),\|\cdot\|_{\infty}\right)$ such that $\left(\mathbf{P}_{1}^{f}\right)_{t} h(x)=\mathbb{E}_{x}\left(h\left(X_{1}^{f}(t)\right)\right)$ for all $x \in \mathcal{S}$ and $h \in \mathcal{C}_{b}(\mathcal{S})$. Our proof of the proposition also shows that if $f(\cdot)$ is constant on $\mathcal{S}$ then $\overline{\mathcal{L}}_{1}^{f}=\overline{\mathcal{L}}$ is merely the closure of $\mathcal{L}$ for the Hilbert space $L_{2}\left(\mu^{0}\right)$.

Proof: Fixing $f \in \mathcal{G}$, we construct the continuous time, homogeneous Markov process $X_{1}^{f}$ on the Hilbert space $\mathcal{H}=L_{2}\left(\mu^{f}\right)$. To this end, consider the bi-linear form

$$
\mathcal{E}_{f}(h, g):=\left\langle h\left(-\mathcal{L}_{1}^{f}\right) g\right\rangle_{\mu^{f}}, \quad \forall g, h \in \mathcal{D}_{f},
$$

where $\mathcal{D}_{f}:=\left\{g: e^{f} g \in \mathcal{D}(\mathcal{L})\right\} \cap \mathcal{D}(\mathcal{L})$ is a linear subspace of $\mathcal{H}$ and $\mathcal{L}_{1}^{f} g:=$ $\mathcal{L} g+\frac{1}{2} e^{-f} \boldsymbol{\Gamma}\left(e^{f}, g\right)$ is a linear operator from $\mathcal{D}_{f}$ to $\mathcal{H}$. Our assumptions imply that the algebra $\mathcal{G}$ is a subspace of $\mathcal{D}_{f}$, hence the latter is dense in $\mathcal{H}$. Recall that $\mathbf{P}_{t}$ is 
$\mu^{0}$-symmetric, so the same applies for its generator $\mathcal{L}$ (i.e. $\langle h \mathcal{L} g\rangle_{\mu^{0}}=\langle g \mathcal{L} h\rangle_{\mu^{0}}$ for all $g, h \in \mathcal{D}(\mathcal{L}))$. It is easy to check that thus $\mathcal{L}_{1}^{f}$ is $\mu^{f}$-symmetric and consequently, $\mathcal{E}_{f}(\cdot, \cdot)$ is a symmetric form on $\mathcal{D}_{f} \times \mathcal{D}_{f}$. Further, by the definition of $\mathcal{L}$ and the $\mu^{0}$-symmetry of $\mathbf{P}_{t}$ we find that for any $g \in \mathcal{D}_{f}$,

$$
\mathcal{E}_{f}(g, g)=\frac{1}{2}\left\langle g^{2} \mathcal{L} e^{f}\right\rangle_{\mu^{0}}-\frac{1}{2}\left\langle g e^{f} \mathcal{L} g\right\rangle_{\mu^{0}}-\frac{1}{2}\left\langle g \mathcal{L}\left(e^{f} g\right)\right\rangle_{\mu^{0}}=\lim _{t \rightarrow 0} \mathcal{E}_{f, t}(g),
$$

where for any $t>0$

$$
\mathcal{E}_{f, t}(g):=\frac{1}{2 t}\left\langle g^{2}-2 g \mathbf{P}_{t} g+\mathbf{P}_{t} g^{2}\right\rangle_{\mu^{f}}=\frac{1}{2 t}\left\langle\mathbb{E}_{x}\left[(g(X(t))-g(x))^{2}\right]\right\rangle_{\mu^{f}} \geq 0,
$$

and consequently $\mathcal{E}_{f}(g, g) \geq 0$ as well. The non-negative quadratic form $\mathcal{E}_{f}$ on the dense subspace $\mathcal{D}\left[\mathcal{E}_{f}\right]=\mathcal{D}_{f}$, is then closable (c.f. [2, Example I.1.3.4]). The closure $\overline{\mathcal{E}}_{f}$ of $\mathcal{E}_{f}$ determines a unique strongly continuous semi-group $\left(\overline{\mathbf{P}}_{1}^{f}\right)_{t}$ of self-adjoint contractions on $\mathcal{H}$ (c.f. 2, Proposition I.1.2.3]). The generator $\overline{\mathcal{L}}_{1}^{f}$ of $\left(\overline{\mathbf{P}}_{1}^{f}\right)_{t}$ is (up to a sign inversion) the Friedrichs extension of $-\mathcal{L}_{1}^{f}$, that is, a non-positive self-adjoint operator on $\mathcal{H}$ satisfying (3.3) for all $g \in \mathcal{D}_{f}$ (c.f. [2, Example I.1.3.4]).

For $\epsilon>0$ let $\varphi_{\epsilon}: \mathbb{R} \rightarrow[-\epsilon, 1+\epsilon]$ be infinitely differentiable functions, such that $\varphi_{\epsilon}(t)=t$ for $t \in[0,1]$ and $0 \leq \varphi_{\epsilon}\left(t_{2}\right)-\varphi_{\epsilon}\left(t_{1}\right) \leq t_{2}-t_{1}$ if $t_{1} \leq t_{2}$ (see [9, Problem I.1.2.1] for a construction of such functions). Obviously, for any $\epsilon, t>0, g \in \mathcal{D}\left(\mathbf{P}_{t}\right)$ and $\mu^{f}$ almost every $x \in \mathcal{S}$,

$$
\mathbb{E}_{x}\left[\left(\varphi_{\epsilon}\left(g(X(t))-\varphi_{\epsilon}(g(x))\right)^{2}\right] \leq \mathbb{E}_{x}\left[(g(X(t))-g(x))^{2}\right] .\right.
$$

By (3.6) this implies that $\mathcal{E}_{f, t}\left(\varphi_{\epsilon}(g)\right) \leq \mathcal{E}_{f, t}(g)$. Further, recall that when $g \in \mathcal{G}$ also $\varphi_{\epsilon}(g) \in \mathcal{G} \subseteq \mathcal{D}_{f}$, in which case it follows from (3.5) that $\mathcal{E}_{f}\left(\varphi_{\epsilon}(g), \varphi_{\epsilon}(g)\right) \leq \mathcal{E}_{f}(g, g)$. As this holds for all $\epsilon>0$ and on the dense subset $\mathcal{G}$ of $\mathcal{D}\left[\overline{\mathcal{E}}_{f}\right]$, we conclude that $\overline{\mathcal{E}}_{f}$ is a symmetric Dirichlet form in $\mathcal{H}$ (c.f. 21, Proposition I.4.10]). Consequently, the strongly continuous semi-group $\left(\overline{\mathbf{P}}_{1}^{f}\right)_{t}$ of self-adjoint contractions on $\mathcal{H}$ is subMarkovian (c.f. [2, Proposition I.3.2.1]), and in particular $\mu^{0}$-almost everywhere $\left(\overline{\mathbf{P}}_{1}^{f}\right)_{t} \psi \geq 0$ whenever $\mu^{0}$-almost everywhere $\psi \geq 0$ (c.f. [2, Definitions I.2.1.1 and I.2.4.1]). Recall that $\mathbf{1} \in \mathcal{G}$ and $\mathcal{L} \mathbf{1}=\mathbf{0}$ (since $\mathbf{P}_{t}$ is Markovian), implying by (3.3) that $\overline{\mathcal{L}}_{1}^{f} \mathbf{1}=\mathbf{0}$ as well. Consequently, $\left(\overline{\mathbf{P}}_{1}^{f}\right)_{t}=e^{t \overline{\mathcal{L}}_{1}^{f}}$ (c.f. [2, Proposition I.1.2.1]), is Markovian as claimed (that is, also $\left(\overline{\mathbf{P}}_{1}^{f}\right)_{t} \mathbf{1}=\mathbf{1}$ for all $t>0$ ).

In carrying out the construction of Langevin dynamics in a non-symmetric setting we use the following analog of Proposition 3.1 which applies in the Hilbert space $L_{2}\left(\mu^{f}\right)$ for any $\mu^{0}$-symmetric Markov semi-group $\overline{\mathbf{P}}_{t}$ on $L_{2}\left(\mu^{0}\right)$ (possibly no longer defined point-wise, and even when the corresponding Markov process is neither strong Markov, nor has right continuous sample path).

Proposition 3.4. If $\overline{\mathbf{P}}_{t}$ is $\mu^{0}$-symmetric Markov semi-group on the Hilbert space $L_{2}\left(\mu^{0}\right)$ with a generator $\overline{\mathcal{L}}$, then Assumption 2.5 holds for a $\mu^{f}$-symmetric, strongly continuous Markov semi-group $\left(\overline{\mathbf{P}}_{0}^{f}\right)_{t}$ on $\mathcal{B}=L_{2}\left(\mu^{f}\right)$, whose generator $\overline{\mathcal{L}}_{0}^{f}$ has the same domain as $\overline{\mathcal{L}}$ and is such that

$$
\overline{\mathcal{L}}_{0}^{f} g=e^{-f} \overline{\mathcal{L}} g, \quad \forall g \in \mathcal{D}(\overline{\mathcal{L}}) .
$$

Proof: Since $\overline{\mathbf{P}}_{t}$ is a $\mu^{0}$-symmetric sub-Markovian semi-group on $L_{2}\left(\mu^{0}\right)$, with domain that is dense in $L_{2}\left(\mu^{0}\right)$ (on account of $\mathcal{G} \subseteq \mathcal{D}(\overline{\mathcal{L}})$ being dense in $L_{2}\left(\mu^{0}\right)$ ), its generator $\overline{\mathcal{L}}$ is a Dirichlet operator, namely, a (negative), self-adjoint operator 
on $L_{2}\left(\mu^{0}\right)$ of a dense domain on which $\left\langle(h-1)_{+} \overline{\mathcal{L}} h\right\rangle_{\mu^{0}} \leq 0$ (c.f. 2, Proposition I.3.2.1]). Fixing $f \in \mathcal{G}$, the linear map $\overline{\mathcal{L}}_{0}^{f}$ from the dense subset $\mathcal{D}(\overline{\mathcal{L}})$ of $L_{2}\left(\mu^{f}\right)$ to $L_{2}\left(\mu^{f}\right)$ given by (3.7) is a symmetric operator on $L_{2}\left(\mu^{f}\right)$, as

$$
\left\langle h_{1} \overline{\mathcal{L}}_{0}^{f} h_{2}\right\rangle_{\mu^{f}}=\left\langle h_{1} \overline{\mathcal{L}} h_{2}\right\rangle_{\mu^{0}}=\left\langle h_{2} \overline{\mathcal{L}} h_{1}\right\rangle_{\mu^{0}}=\left\langle h_{2} \overline{\mathcal{L}}_{0}^{f} h_{1}\right\rangle_{\mu^{f}}
$$

for all $h_{1}, h_{2} \in \mathcal{D}(\overline{\mathcal{L}})$. Further, if $h, \phi \in L_{2}\left(\mu^{f}\right)$ are such that $\left\langle h \overline{\mathcal{L}}_{0}^{f} g\right\rangle_{\mu^{f}}=\langle\phi g\rangle_{\mu^{f}}$ for all $g \in \mathcal{D}\left(\overline{\mathcal{L}}_{0}^{f}\right)=\mathcal{D}(\overline{\mathcal{L}})$ then of course $\langle h \overline{\mathcal{L}} g\rangle_{\mu^{0}}=\left\langle e^{f} \phi g\right\rangle_{\mu^{0}}$ for $h, \phi \in L_{2}\left(\mu^{0}\right)$. With $\overline{\mathcal{L}}$ self-adjoint on $L_{2}\left(\mu^{0}\right)$ this implies that $h \in \mathcal{D}(\overline{\mathcal{L}})$ and $\phi=e^{-f} \overline{\mathcal{L}} h=\overline{\mathcal{L}}_{0}^{f} h$, namely, the symmetric operator $\overline{\mathcal{L}}_{0}^{f}$ is self-adjoint on $L_{2}\left(\mu^{f}\right)$. Clearly,

$$
\left\langle(h-1)_{+} \overline{\mathcal{L}}_{0}^{f} h\right\rangle_{\mu^{f}}=\left\langle(h-1)_{+} \overline{\mathcal{L}} h\right\rangle_{\mu^{0}} \leq 0
$$

for all $h \in \mathcal{D}(\overline{\mathcal{L}})=\mathcal{D}\left(\overline{\mathcal{L}}_{0}^{f}\right)$. We thus deduce that $\overline{\mathcal{L}}_{0}^{f}$ is a Dirichlet operator on $L_{2}\left(\mu^{f}\right)$, hence the generator of a $\mu^{f}$-symmetric sub-Markovian semi-group on $L_{2}\left(\mu^{f}\right)$, denoted hereafter $\left(\overline{\mathbf{P}}_{0}^{f}\right)_{t}$ (for example, apply again [2, Proposition I.3.2.1], now in the converse direction). Finally, with $\overline{\mathbf{P}}_{t}$ a Markov semi-group, we have that $\mathbf{1} \in \mathcal{D}(\overline{\mathcal{L}})$ and $\mu^{0}$-a.s. $\overline{\mathcal{L}} \mathbf{1}=\mathbf{0}$. Of course, the same applies $\mu^{f}$-a.s. for $\overline{\mathcal{L}}_{0}^{f}$ of (3.7). Consequently, $\left(\overline{\mathbf{P}}_{0}^{f}\right)_{t}$ is actually a $\mu^{f}$-symmetric Markov semi-group on $L_{2}\left(\mu^{f}\right)$ (e.g. [2, Exercise I.3.1]), as claimed.

Langevin dynamics in a non-symmetric setting. Building on Propositions 3.1 and 3.2 we shall construct a generalized Langevin dynamics when $\mu^{0}$ is invariant for the strongly continuous Markov semi-group $\mathbf{P}_{t}$ on $\mathcal{D}\left(\mathbf{P}_{t}\right)$ (of a strong Markov process of right continuous sample path $X(t)$ valued on a complete, separable metric space $\mathcal{S}$ ), now in case $\mathbf{P}_{t}$ is not $\mu^{0}$-symmetric. This construction is more complex than what we have seen in Proposition 3.2 and it involves certain (mild) restrictions on the domains of various generators. We start by letting $\overline{\mathbf{P}}_{t}$ denote the extension of $\mathbf{P}_{t}$ to a $\mu^{0}$-invariant strongly continuous, Markov semi-group on the Hilbert space $L_{2}\left(\mu^{0}\right)$ and $\overline{\mathbf{P}}_{t}^{\star}$ the adjoint of $\overline{\mathbf{P}}_{t}$ in $L_{2}\left(\mu^{0}\right)$. The adjoint semi-group $\left(\overline{\mathbf{P}}_{t}^{\star}\right)_{t \geq 0}$ is then strongly continuous in $L_{2}\left(\mu^{0}\right)$ and its generator $\overline{\mathcal{L}}^{\star}$ is the adjoint of the generator $\overline{\mathcal{L}}$ of $\overline{\mathbf{P}}_{t}$ (see [10, Theorem 4.3]). Further, since $\overline{\mathbf{P}}_{t}$ is $\mu^{0}$-invariant and Markovian on $L_{2}\left(\mu^{0}\right)$, the same applies for $\overline{\mathbf{P}}_{t}^{\star}$.

Let $\overline{\boldsymbol{\Gamma}}(h, g)=\overline{\mathcal{L}}(g h)-h \overline{\mathcal{L}}(g)-g \overline{\mathcal{L}}(h)$ denote the $L_{2}\left(\mu^{0}\right)$ extension of $\boldsymbol{\Gamma}$. We seek a perturbed Markovian semi-group whose generator has the form

$$
\overline{\mathcal{L}}_{1}^{f}=\widetilde{\mathcal{L}}+\frac{1}{2} e^{-f} \overline{\boldsymbol{\Gamma}}\left(e^{f}, \cdot\right)+e^{-f} \mathcal{L}_{a}
$$

where $\widetilde{\mathcal{L}}=\frac{1}{2}\left(\overline{\mathcal{L}}+\overline{\mathcal{L}}^{\star}\right)$ and $\mathcal{L}_{a}=\frac{1}{2}\left(\overline{\mathcal{L}}-\overline{\mathcal{L}}^{\star}\right)$ correspond to the symmetric and antisymmetric parts of $\overline{\mathcal{L}}$, respectively. Indeed, it is easy to see that $\overline{\mathcal{L}}_{1}^{f}$ is $\mu^{f}$-invariant and $\overline{\mathcal{L}}_{1}^{\delta f} \rightarrow \mathcal{L}$ for $\delta \downarrow 0$. However, while $\widetilde{\mathcal{L}}$ is a generator of a Markovian semi-group (as shown in Corollary [3.7), this is not the case for $\mathcal{L}_{a}$. Hence, we alternatively construct $\overline{\mathcal{L}}_{1}^{f}$ in Proposition 3.8 as the sum of the Markovian generators $\overline{\mathcal{L}}_{0}^{f}$ of Proposition 3.4 and $\widetilde{\mathcal{L}}^{f}$ which we construct next.

Lemma 3.5. Suppose $\mathbf{P}_{t}$ is a $\mu^{0}$-invariant strongly continuous Markov semi-group. Assume further that $\mathcal{G} \subseteq \mathcal{D}\left(\overline{\mathcal{L}}^{\star}\right)$ and $\overline{\mathcal{L}}^{\star} g \in \mathcal{G}$ for all $g \in \mathcal{G}$. Then, for each nonnegative $f \in \mathcal{G}$ there exists a Markov process $\widetilde{X}^{f}$ such that the generator of its 
$\mu^{f}$-symmetric, strongly continuous semi-group $\widetilde{\mathbf{P}}_{t}^{f}$ on the Hilbert space $\mathcal{B}=L_{2}\left(\mu^{f}\right)$, satisfies

$$
\widetilde{\mathcal{L}}^{f} g=\left(1-e^{-f}\right)\left(\frac{1}{2} \overline{\mathcal{L}} g+\frac{1}{2} \overline{\mathcal{L}}^{\star} g\right)+\frac{1}{2} e^{-f} \overline{\boldsymbol{\Gamma}}\left(e^{f}, g\right), \quad \forall g \in \mathcal{G}
$$

and for which Assumption 2.5 holds.

Proof: Fixing a non-negative $f \in \mathcal{G}$, consider the linear subspace

$$
\widehat{\mathcal{D}}_{f}:=\left\{h: e^{f} h \in \mathcal{D}(\overline{\mathcal{L}})\right\} \cap \mathcal{D}(\overline{\mathcal{L}}) \cap \mathcal{D}\left(\overline{\mathcal{L}}^{\star}\right)
$$

of $\mathcal{H}=L_{2}\left(\mu^{f}\right)$ and the linear operator $\widehat{\mathcal{L}}^{f}$ from $\widehat{\mathcal{D}}_{f}$ to $\mathcal{H}$, defined via (3.8). It is not hard to verify that $\widehat{\mathcal{L}}^{f}$ is $\mu^{f}$-symmetric operator on $\widehat{\mathcal{D}}_{f}$, with the associated symmetric bi-linear form

$$
\begin{aligned}
\widehat{\mathcal{E}}_{f}(h, g) & :=-\left\langle h \widehat{\mathcal{L}}^{f} g\right\rangle_{\mu^{f}} \\
& =\frac{1}{2}\left[\left\langle h \overline{\mathcal{L}}\left(g-g e^{f}\right)\right\rangle_{\mu^{0}}+\left\langle g \overline{\mathcal{L}}\left(h-h e^{f}\right)\right\rangle_{\mu^{0}}+\left\langle g h \overline{\mathcal{L}} e^{f}\right\rangle_{\mu^{0}}\right]
\end{aligned}
$$

Since $\overline{\mathcal{L}}$ and its adjoint $\overline{\mathcal{L}}^{\star}$ are the generators of the $\mu^{0}$-invariant, strongly continuous semi-groups $\overline{\mathbf{P}}_{t}$ and $\overline{\mathbf{P}}_{t}^{\star}$ on $L_{2}\left(\mu^{0}\right)$, similarly to (3.5), we further have that for any $g \in \widehat{\mathcal{D}}_{f}$

$$
\widehat{\mathcal{E}}_{f}(g, g)=\frac{1}{2}\left\langle g^{2} \overline{\mathcal{L}} e^{f}\right\rangle_{\mu^{0}}+\langle g \overline{\mathcal{L}} g\rangle_{\mu^{0}}-\left\langle g \overline{\mathcal{L}}\left(e^{f} g\right)\right\rangle_{\mu^{0}}=\lim _{t \rightarrow 0} \widehat{\mathcal{E}}_{f, t}(g)
$$

where for any $t>0$,

$$
\widehat{\mathcal{E}}_{f, t}(g):=\frac{1}{2 t}\left\langle\left(e^{f}-1\right)\left[g^{2}-2 g \overline{\mathbf{P}}_{t}^{\star} g+\overline{\mathbf{P}}_{t}^{\star} g^{2}\right]\right\rangle_{\mu^{0}} \geq 0
$$

in view of the non-negativity of $f$. Hence, $\widehat{\mathcal{E}}_{f}(g, g) \geq 0$ for all $g \in \widehat{\mathcal{D}}_{f}$, and since by our assumptions $\mathcal{G} \subseteq \widehat{\mathcal{D}}_{f}$, we conclude that the non-negative quadratic form $\widehat{\mathcal{E}}_{f}$ of dense domain $\widehat{\mathcal{D}}_{f}$ is closable, denoting by $\widetilde{\mathcal{E}}_{f}$ its closure, and by $\widetilde{\mathbf{P}}_{t}$ and $\widetilde{\mathcal{L}}^{f}$ the corresponding strongly continuous semi-group and generator. Since $\overline{\mathbf{P}}_{t}^{\star}$ is a Markov semi-group, by the same argument as in the proof of Proposition 3.2 we deduce that replacing $g$ by $\varphi_{\epsilon}(g)$ reduces the value of $g^{2}-2 g \overline{\mathbf{P}}_{t}^{\star} g+\overline{\mathbf{P}}_{t}^{\star} g^{2} \geq 0$, implying that $\widehat{\mathcal{E}}_{f, t}\left(\varphi_{\epsilon}(g)\right) \leq \widehat{\mathcal{E}}_{f, t}(g)$ for all $\epsilon, t>0$ and $g \in \mathcal{G}$, a dense subset of $\mathcal{D}\left[\widetilde{\mathcal{E}}_{f}\right]$. Arguing again as in Proposition 3.2, we conclude that $\widetilde{\mathcal{E}}_{f}$ is a symmetric Dirichlet form in $\mathcal{H}$ with $\widetilde{\mathbf{P}}_{t}=e^{t \widetilde{\mathcal{L}}^{f}}$ a strongly continuous Markovian semi-group (recall that $\mathbf{1} \in \mathcal{G}$ and $\left.\overline{\mathcal{L}} \mathbf{1}=\overline{\mathcal{L}}^{\star} \mathbf{1}=\mathbf{0}\right)$.

Remark 3.6. Though Assumption 2.5 holds for the $\mu^{f}$-symmetric Markov semigroup $\widetilde{\mathbf{P}}_{t}^{f}$, the Fluctuation Dissipation Theorem 2.10 fails in this case, for $\widetilde{\mathcal{L}}^{\delta f}$ of (3.8) converges to zero as $\delta \downarrow 0$ (and not to $\mathcal{L}$ ). We thus need the additional ingredients of Proposition 3.8 in order to complete the construction of the Langevin dynamics in non-symmetric setting.

Considering Lemma 3.5 for $f=\mathbf{1}$ and then Proposition 3.4 for $f=\log \left(1-e^{-1}\right) \mathbf{1}$ we get the existence of the following symmetric Markov process.

Corollary 3.7. For any $\mu^{0}$-invariant strongly continuous Markov semi-group $\mathbf{P}_{t}$, if $\mathcal{G} \subseteq \mathcal{D}\left(\overline{\mathcal{L}}^{\star}\right)$ and $\overline{\mathcal{L}}^{\star} g \in \mathcal{G}$ for all $g \in \mathcal{G}$, then Assumption 2.5 holds for a Markov 
process $\widetilde{X}$ on the Hilbert space $L_{2}\left(\mu^{0}\right)$. The generator of its $\mu^{0}$-symmetric, strongly continuous semi-group $\widetilde{\mathbf{P}}_{t}$ is such that for any $g \in \mathcal{D}(\overline{\mathcal{L}}) \cap \mathcal{D}\left(\overline{\mathcal{L}}^{\star}\right)$,

$$
\widetilde{\mathcal{L}} g=\frac{1}{2} \overline{\mathcal{L}} g+\frac{1}{2} \overline{\mathcal{L}}^{\star} g .
$$

We complete our construction by adding to the generator of Lemma 3.5 an appropriate non-symmetric perturbation (taken from Proposition 3.1).

Proposition 3.8. Suppose as in Lemma 3.5 that $f \in \mathcal{G}$ is non-negative and $\overline{\mathcal{L}}^{\star}(\mathcal{G}) \subseteq \mathcal{G}$. Further assume that $\mathcal{D}(\overline{\mathcal{L}})$ is contained in the domain of the generator $\widetilde{\mathcal{L}}^{f}$ of $\widetilde{X}^{f}$. Then, Assumption [2.5 holds for a $\mu^{f}$-invariant Markov process $X_{1}^{f}$ on $\mathcal{B}=L_{2}\left(\mu^{f}\right)$. The generator $\overline{\mathcal{L}}_{1}^{f}$ of its strongly continuous semi-group $\left(\overline{\mathbf{P}}_{1}^{f}\right)_{t}$ has the same domain as $\overline{\mathcal{L}}$ and is such that

$$
\overline{\mathcal{L}}_{1}^{f}=\widetilde{\mathcal{L}}^{f}+e^{-f} \overline{\mathcal{L}} .
$$

Remark 3.9. Proposition 3.8 is the non-symmetric generalization of Proposition 3.2 since if $\mathbf{P}_{t}$ is $\mu^{0}$-symmetric then $\overline{\mathcal{L}}^{\star}=\overline{\mathcal{L}}$ and the operator of (3.14) and the corresponding Markov process coincide with $\overline{\mathcal{L}}_{1}^{f}$ (and $X_{1}^{f}$ ) of Proposition 3.2. Also note that since each $f \in \mathcal{G}$ is bounded below, you have the non-negativity condition of the proposition by adding to any given $f \in \mathcal{G}$ a sufficiently large constant, without changing the corresponding normalized invariant measure $\mu^{f} / \mu^{f}(\mathcal{S})$.

Proof: From Proposition 3.1 we get the Markov process $X_{0}^{f}(t)$ on $\left(\mathcal{C}_{b}(\mathcal{S}),\|\cdot\|_{\infty}\right)$ whose semi-group is generated by $\mathcal{L}_{0}^{f}=e^{-f} \mathcal{L}$ and is invariant for $\mu^{f}$. Let $\overline{\mathbf{P}}_{0}^{f}$ denote the extension of this semi-group to $L_{2}\left(\mu^{f}\right)$ and $\overline{\mathcal{L}}_{0}^{f}$ its generator. Since $f$ is bounded it is easy to see that $\overline{\mathcal{L}}_{0}^{f}=e^{-f} \overline{\mathcal{L}}$ has the same domain as $\overline{\mathcal{L}}$. Subject to the assumptions of Lemma 3.5 we get the $\mu^{f}$-symmetric Markov semi-group $\widetilde{\mathbf{P}}_{t}^{f}$ on the same Hilbert space, whose generator $\widetilde{\mathcal{L}}^{f}$ satisfies (3.8). In particular, by Hille-Yosida theorem we know that $\widetilde{\mathcal{L}}^{f}$ is a closed operator, so our assumption that its domain contains the domain of $\overline{\mathcal{L}}$ implies that the operator $\overline{\mathcal{L}}_{1}^{f}($ on $\mathcal{D}(\overline{\mathcal{L}}))$, given by (3.14) is a generator of a strongly continuous semi-group $\overline{\mathbf{P}}_{1}^{f}$ (c.f. [5], pages 631-639]). Further, with $e^{f} \in \mathcal{G}$ (an algebra) such that both $\mathcal{L}(\mathcal{G}) \subseteq \mathcal{G}$ and $\overline{\mathcal{L}}^{\star}(\mathcal{G}) \subseteq \mathcal{G}$, we have the same properties for $\overline{\mathcal{L}}_{0}^{f}$ and $\widetilde{\mathcal{L}}^{f}$, hence for $\overline{\mathcal{L}}_{1}^{f}$ as well. The latter operator is the sum of the generators of two $\mu^{f}$-invariant Markov semigroups, so by Trotter's product formula (c.f. [10, Theorem 8.12]), we conclude that the corresponding generated semi-group is both Markovian and $\mu^{f}$-invariant.

\section{The CORRESPONDING RESPONSE FUnCTIONS}

The response function is not determined just by the given Markov process $X(\cdot)$, its invariant measure $\mu^{0}$, and the perturbation function $f \in \mathcal{G}$. Indeed, Theorem 4.1 provides the response function of the Markovian perturbations of Propositions 3.1 and 3.4 while Theorem 4.2 provides the response function for the Markovian perturbation of Proposition 3.8 under the uniform control of (4.1) on the antisymmetric part of the generator $\overline{\mathcal{L}}$. See also Corollary 4.6 for the simpler response function in the symmetric case of Proposition 3.2. Typically the response functions in these two theorems are not the same. 
Theorem 4.1. Taking $(\mathcal{B},\|\cdot\|)=\left(\mathcal{C}_{b}(\mathcal{S}),\|\cdot\|_{\infty}\right)$, the Markov perturbation $X_{0}^{f}(t)$ has a response function of the form 2.7), with $\mathbf{A}_{f}=\left(\mathbf{A}_{0}\right)_{f}=-f \mathcal{L}$.

Proof: Recall that $X_{0}^{f}(t)$ satisfies Assumption 2.5 for $(\mathcal{B},\|\cdot\|)=\left(\mathcal{C}_{b}(\mathcal{S}),\|\cdot\|_{\infty}\right)$. Fixing $f \in \mathcal{G}$, observe that $\mathcal{D}\left(\left(\mathbf{A}_{0}\right)_{f}\right)=\mathcal{D}(-f \mathcal{L})=\mathcal{D}(\mathcal{L})$ which contains $\widehat{\mathcal{G}}$ by our assumptions about $\mathcal{G}$. Further, by strong continuity of $\mathbf{P}_{s}$ on $\left(\mathcal{D}\left(\mathbf{P}_{t}\right),\|\cdot\|_{\infty}\right)$ it follows that for each given $g \in \mathcal{G}$,

$$
\left(\mathbf{A}_{0}\right)_{f} \mathbf{P}_{s} g=-f \mathcal{L} \mathbf{P}_{s} g=-f \mathbf{P}_{s}(\mathcal{L} g),
$$

is also strongly continuous. Fixing $\widehat{g}=\mathbf{P}_{v} g$ for $v \geq 0$ and $g \in \mathcal{G}$, and taking $\mathbf{P}_{t}^{f}=$ $\left(\mathbf{P}_{0}^{f}\right)_{t}$, as in the proof of Proposition 2.9] it suffices to show that $\left\|\zeta_{t}^{\delta}\right\|_{\infty} \rightarrow 0$ as $\delta \downarrow 0$, uniformly over $t \in(0, T]$, with $\zeta_{t}^{\delta}$ given by (2.12). To this end, since $\mathcal{L} \mathbf{P}_{u}=\mathbf{P}_{u} \mathcal{L}$, $\mathcal{L}_{0}^{f}=e^{-f} \mathcal{L}$ and $\left(\mathbf{A}_{0}\right)_{f}=-f \mathcal{L}$, it is not hard to verify that $\zeta_{t}^{\delta}=\phi_{\delta} \mathcal{L} r_{t}-\psi_{-\delta} \mathbf{P}_{t} \widehat{h}$, where $\widehat{h}=\mathbf{P}_{v} h$ for $h=\mathcal{L} g \in \mathcal{G}$, the continuous functions $\phi_{\delta}:=\mathbf{1}-e^{-\delta f}$ and $\psi_{\delta}:=\delta^{-1}\left(e^{\delta f}-\delta f-\mathbf{1}\right)$ are such that $\left\|\phi_{\delta}\right\|_{\infty}+\left\|\psi_{-\delta}\right\|_{\infty} \rightarrow 0$ as $\delta \downarrow 0$, and $r_{t}=\int_{0}^{t} \mathbf{P}_{t-u} f \mathbf{P}_{u} \widehat{h} d u$ is in $\mathcal{D}(\mathcal{L})$ by our assumptions about the space $\mathcal{G}$ of test functions.

With $\mathbf{P}_{t}$ contractive on $\left(\mathcal{D}\left(\mathbf{P}_{t}\right),\|\cdot\|_{\infty}\right)$, we thus have that for any $t \in[0, T]$,

$$
\left\|\zeta_{t}^{\delta}\right\|_{\infty} \leq\left\|\phi_{\delta}\right\|_{\infty}\left\|\mathcal{L} r_{t}\right\|_{\infty}+\left\|\psi_{-\delta}\right\|_{\infty}\|h\|_{\infty} \leq \eta_{\delta}(f, g, T),
$$

with $\eta_{\delta} \rightarrow 0$ as $\delta \downarrow 0$, provided $\sup \left\{\left\|\mathcal{L} r_{s}\right\|_{\infty}: s \leq T\right\}<\infty$.

To show the latter, recall that

$$
\begin{aligned}
\mathcal{L} r_{s}+f \mathbf{P}_{s} \widehat{h} & =\partial_{s} r_{s}=\partial_{s}\left(\int_{0}^{s} \mathbf{P}_{u} f \mathbf{P}_{s-u} \widehat{h} d u\right) \\
& =\mathbf{P}_{s} f \widehat{h}+\int_{0}^{s} \mathbf{P}_{u} f \mathcal{L} \mathbf{P}_{s-u} \widehat{h} d u=\mathbf{P}_{s} f \widehat{h}+\int_{0}^{s} \mathbf{P}_{u} f \mathbf{P}_{s-u} \mathcal{L} \widehat{h} d u,
\end{aligned}
$$

hence by contractivity of the semi-group $\mathbf{P}_{u}$,

$$
\begin{aligned}
\left\|\mathcal{L} r_{s}\right\|_{\infty} & \leq\left\|f \mathbf{P}_{s} \widehat{h}\right\|_{\infty}+\left\|\mathbf{P}_{s} f \widehat{h}\right\|_{\infty}+\int_{0}^{s}\left\|\mathbf{P}_{u} f \mathbf{P}_{s-u} \mathcal{L} \widehat{h}\right\|_{\infty} d u \\
& \leq 2\|f\|_{\infty}\|h\|_{\infty}+T\|f\|_{\infty}\|\mathcal{L} h\|_{\infty}
\end{aligned}
$$

which completes the proof.

Theorem 4.2. Suppose in addition to the assumptions of Proposition 3.8 holding for $\delta f$ and all $\delta \in(0,1]$, that $\left(\overline{\mathbf{P}}_{1}^{\delta f}\right)_{t} \widehat{g} \in \mathcal{D}\left(\overline{\mathcal{L}}^{\star}\right)$ for all $\widehat{g} \in \widehat{\mathcal{G}}, t \geq 0$, and that $\mathcal{D}(\overline{\mathcal{L}}) \subseteq \mathcal{D}(\widetilde{\mathcal{L}})$ for $\widetilde{\mathcal{L}}$ of Corollary 3.7 , with

$$
\|\widetilde{\mathcal{L}} r-\overline{\mathcal{L}} r\|^{2} \leq-K\langle r \overline{\mathcal{L}} r\rangle_{\mu^{0}}
$$

for some $K<\infty$ and all $r \in \mathcal{D}(\overline{\mathcal{L}})$. The semi-groups $\left(\overline{\mathbf{P}}_{1}^{f}\right)_{t}$ for the Markovian perturbations $X_{1}^{f}(t)$ on $\mathcal{B}=L_{2}\left(\mu^{0}\right)$ then have a response function of the form 2.7), now with

$$
\mathbf{A}_{f}=\left(\mathbf{A}_{1}\right)_{f}=\frac{1}{2} \overline{\boldsymbol{\Gamma}}(f, \cdot)+f \widetilde{\mathcal{L}}-f \overline{\mathcal{L}}
$$

a linear operator on $\mathcal{D}\left(\left(\mathbf{A}_{1}\right)_{f}\right):=\{g: f g \in \mathcal{D}(\overline{\mathcal{L}})\} \cap \mathcal{D}(\overline{\mathcal{L}})$. 
Remark 4.3. Since $\left(\mathbf{A}_{1}\right)_{f}$ is applied in (2.7) only on functions in the set $\widehat{\mathcal{G}}=$ $\left\{\mathbf{P}_{t} g: g \in \mathcal{G}, t \geq 0\right\}$, it is not hard to verify that 4 .2. is valid even with $\overline{\mathcal{L}}$ and $\overline{\boldsymbol{\Gamma}}$ replaced by $\mathcal{L}$ and $\boldsymbol{\Gamma}$, and that only the term corresponding to $f \widetilde{\mathcal{L}}$ in (2.7) might not be in $\mathcal{C}_{b}(\mathcal{S})$ (hence requiring the application of $\overline{\mathbf{P}}_{s}$ instead of $\mathbf{P}_{s}$ ).

Remark 4.4. Of the conditions of Theorem 4.2, it is the least convenient to check that $r=\left(\overline{\mathbf{P}}_{1}^{\delta f}\right)_{t} \widehat{g}$ is in the domain of $\overline{\mathcal{L}}^{\star}$. However, we use it only once, to deduce that then $\langle r \overline{\mathcal{L}} r\rangle_{\mu^{0}}=\langle r \widetilde{\mathcal{L}} r\rangle_{\mu^{0}}$. Thus, one can eliminate the former condition whenever there is a direct way to verify the latter.

Heuristically, the condition (4.1) tells us that the symmetric part of the dynamics is dominant, in the same spirit as our assumption that $\mathcal{D}(\overline{\mathcal{L}})$ is contained in both $\mathcal{D}(\widetilde{\mathcal{L}})$ and $\mathcal{D}\left(\widetilde{\mathcal{L}}^{f}\right)$ (and many non-symmetric examples have different domains for $\overline{\mathcal{L}}$, $\overline{\mathcal{L}}^{\star}$ and $\left.\widetilde{\mathcal{L}}\right)$. Nevertheless, in many cases we do not have to worry about domains of the various operators and in certain settings arrive at the same conclusion even when (4.1) does not hold (see Section 5 for one such example).

Remark 4.5. Since $\mu^{\delta \mathbf{1}}=\mu^{0}$ for any $\delta>0$, it is natural to choose $\mathbf{P}_{t}^{\delta \mathbf{1}}=\mathbf{P}_{t}$, as is the case for $X_{1}^{f}(\cdot)$, yielding then that $\mathbf{A}_{\mathbf{1}}=\mathbf{0}$. However, this is not always done. For example, $\mathbf{P}_{t}^{\delta \mathbf{1}} \neq \mathbf{P}_{t}$ for $X_{0}^{f}(\cdot)$, where indeed $\left(\mathbf{A}_{0}\right)_{\mathbf{1}}=-\mathcal{L} \neq \mathbf{0}$. Also note that by Definition 2.7 the response function $R_{X^{f}, g}(s, t)$ is always linear in $g$ and homogeneous with respect to multiplication of $f$ by a positive scalar. In Theorems 4.1 and 4.2, the response function is further linear in $f$, but this does not always apply (see Example 5.4 for the Metropolis perturbation in which $\mathbf{A}_{-f} \neq-\mathbf{A}_{f}$ ).

In case of $\mu^{0}$-symmetric processes we get the following corollary upon considering $\widetilde{\mathcal{L}}=\overline{\mathcal{L}}^{\star}=\overline{\mathcal{L}}$. The direct proof of this corollary is of course simpler and shorter than that of Theorem 4.2

Corollary 4.6. Suppose $\mathbf{P}_{t}$ is $\mu^{0}$-symmetric and that for all $\delta \in(0,1]$ the generator of the $\mu^{\delta f}$-symmetric semi-group of Proposition 3.2 is such that $\mathcal{D}(\overline{\mathcal{L}}) \subseteq \mathcal{D}\left(\overline{\mathcal{L}}_{1}^{\delta f}\right)$. These semi-groups then have a response function of the form 2.7) with $\left(\mathbf{A}_{1}\right)_{f}=$ $\frac{1}{2} \boldsymbol{\Gamma}(f, \cdot)$ (whose domain $\{g \in \mathcal{D}(\mathcal{L}): f g \in \mathcal{D}(\mathcal{L})\}$ contains $\widehat{\mathcal{G}}$ ).

Proof of Theorem 4.2: Recall Proposition 3.8 that fixing hereafter non-negative $f \in \mathcal{G}$, Assumption 2.5 holds for the Hilbert space $\mathcal{H}=L_{2}\left(\mu^{0}\right)$ and further, $\widehat{\mathcal{G}}=$ $\left\{\mathbf{P}_{t} g: g \in \mathcal{G}, t \geq 0\right\}$ is in the domain $\mathcal{D}(\mathbf{A})$ of the linear operator $\mathbf{A}=\left(\mathbf{A}_{1}\right)_{f}$ of (4.2). Due to the linearity of $\mathbf{A}$, for the strong continuity of $\mathbf{A P}_{s} g$ in $\mathcal{H}$ per given $g \in \mathcal{G}$, it suffices to show the convergence to zero in $\mathcal{H}$ of $\overline{\mathcal{L}}\left(\mathbf{P}_{s} \widehat{g}-\widehat{g}\right), \boldsymbol{\Gamma}\left(f, \mathbf{P}_{s} \widehat{g}-\widehat{g}\right)$ and $\widetilde{\mathcal{L}}\left(\mathbf{P}_{s} \widehat{g}-\widehat{g}\right)$, when $s \downarrow 0$, per given $\widehat{g} \in \widehat{\mathcal{G}}$. To this end, note first that $\overline{\mathcal{L}}\left(\mathbf{P}_{s} \widehat{g}-\right.$ $\widehat{g})=\left(\mathbf{P}_{s}-\mathbf{I}\right) \mathcal{L} \widehat{g}$ (since $\widehat{g}, \mathbf{P}_{s} \widehat{g} \in \mathcal{D}(\mathcal{L})$ ), with the latter converging to zero in $\mathcal{H}$ by the strong continuity of $\mathbf{P}_{s}$. Further, applying (4.1) for $\mathbf{P}_{s} \widehat{g}-\widehat{g} \in \mathcal{D}(\overline{\mathcal{L}})$, by the preceding argument, the convergence of $\widetilde{\mathcal{L}}\left(\mathbf{P}_{s} \widehat{g}-\widehat{g}\right)$ in $\mathcal{H}$ is a consequence of the convergence to zero of

$$
-\left\langle\left(\mathbf{P}_{s} \widehat{g}-\widehat{g}\right) \overline{\mathcal{L}}\left(\mathbf{P}_{s} \widehat{g}-\widehat{g}\right)\right\rangle_{\mu^{0}}=-\left\langle\left(\mathbf{P}_{s} \widehat{g}-\widehat{g}\right)\left(\mathbf{P}_{s}-\mathbf{I}\right)(\mathcal{L} \widehat{g})\right\rangle_{\mu^{0}} \leq 2\left\|\mathbf{P}_{s} \widehat{g}-\widehat{g}\right\|\|\mathcal{L} \widehat{g}\|,
$$

by the strong continuity of $\mathbf{P}_{s}$.

To deal with the last remaining term, namely $\boldsymbol{\Gamma}\left(f, \mathbf{P}_{s} \widehat{g}-\widehat{g}\right)$, note that if $\left(h_{1}, h_{2}\right) \in$ $\mathcal{D}(\boldsymbol{\Gamma})$ then $\boldsymbol{\Gamma}_{t}\left(h_{1}, h_{2}\right) \rightarrow \boldsymbol{\Gamma}\left(h_{1}, h_{2}\right)$ in supremum norm and hence also in $\mathcal{H}$, when 
$t \downarrow 0$, where

$$
\boldsymbol{\Gamma}_{t}(g, h):=t^{-1}\left[g h-g \mathbf{P}_{t} h-h \mathbf{P}_{t} g+\mathbf{P}_{t} g h\right],
$$

is a bi-linear symmetric, non-negative definite operator.

Since $\boldsymbol{\Gamma}_{t}\left(h_{1}, h_{2}\right)^{2} \leq \boldsymbol{\Gamma}_{t}\left(h_{1}, h_{1}\right) \boldsymbol{\Gamma}_{t}\left(h_{2}, h_{2}\right)$, we have in particular that

$$
\begin{aligned}
\left\|\boldsymbol{\Gamma}\left(f, \mathbf{P}_{s} \widehat{g}-\widehat{g}\right)\right\|^{2} & =\lim _{t \rightarrow 0}\left\|\boldsymbol{\Gamma}_{t}\left(f, \mathbf{P}_{s} \widehat{g}-\widehat{g}\right)\right\|^{2} \\
& \leq \lim _{t \rightarrow 0}\left\|\boldsymbol{\Gamma}_{t}(f, f)\right\|_{\infty}\left\langle\boldsymbol{\Gamma}_{t}\left(\mathbf{P}_{s} \widehat{g}-\widehat{g}, \mathbf{P}_{s} \widehat{g}-\widehat{g}\right)\right\rangle_{\mu^{0}} .
\end{aligned}
$$

As $(f, f) \in \mathcal{D}(\boldsymbol{\Gamma})$ we have the convergence of $\boldsymbol{\Gamma}_{t}(f, f)$ to $\boldsymbol{\Gamma}(f, f)$ in supremum norm. Further, the $\mu^{0}$-invariance of $\mathbf{P}_{s}$ results with

$$
\left\langle\boldsymbol{\Gamma}_{t}\left(\mathbf{P}_{s} \widehat{g}-\widehat{g}, \mathbf{P}_{s} \widehat{g}-\widehat{g}\right)\right\rangle_{\mu^{0}} \rightarrow-2\left\langle\left(\mathbf{P}_{s} \widehat{g}-\widehat{g}\right) \mathcal{L}\left(\mathbf{P}_{s} \widehat{g}-\widehat{g}\right)\right\rangle_{\mu^{0}}
$$

as $t \rightarrow 0$, which as we have already shown converges to zero when $s \rightarrow 0$. Combining all these facts we get the stated strong continuity of $\mathbf{A P}_{s} g$.

Fixing $\widehat{g}=\mathbf{P}_{v} g$ for $v \geq 0$ and $g \in \mathcal{G}$, it thus remains only to show that for $\mathbf{P}_{t}^{\delta}:=\left(\overline{\mathbf{P}}_{1}^{\delta f}\right)_{t}$ of Proposition 3.8 ,

$$
\rho_{t}^{\delta}:=\delta^{-1}\left(\mathbf{P}_{t}^{\delta}-\mathbf{P}_{t}\right) \widehat{g}-\int_{0}^{t} \mathbf{P}_{t-u} \mathbf{A} \mathbf{P}_{u} \widehat{g} d u
$$

is such that $t^{-1}\left\|\rho_{t}^{\delta}\right\| \rightarrow 0$ as $\delta \downarrow 0$ uniformly in $t \in(0, T]$. To this end, recall that the generator $\mathcal{L}^{\delta}:=\overline{\mathcal{L}}_{1}^{\delta f}$ of the semi-group $\mathbf{P}_{t}^{\delta}$ of Proposition 3.8 has exactly the same domain as $\overline{\mathcal{L}}$, so with $\widehat{\mathcal{G}} \subseteq \mathcal{D}(\overline{\mathcal{L}})$, we see that both $\mathbf{P}_{t} \widehat{g}$ and $\mathbf{P}_{t}^{\delta} \widehat{g}$ are in the domain of $\overline{\mathcal{L}}$ (and $\mathcal{L}^{\delta}$ ), with

$$
\psi_{t}^{\delta}=\delta^{-1}\left(\mathcal{L}^{\delta} \mathbf{P}_{t}^{\delta} \widehat{g}-\overline{\mathcal{L}} \mathbf{P}_{t}^{\delta} \widehat{g}\right)-\mathbf{A} \mathbf{P}_{t} \widehat{g},
$$

well defined. Further, as we shall prove at the sequel,

Lemma 4.7. For any $f \in \mathcal{G}, \widehat{g} \in \widehat{\mathcal{G}}$ and $T<\infty$ there exists $\widehat{K}=\widehat{K}(f, \widehat{g}, T)$ finite, such that $\left\|\psi_{t}^{\delta}\right\| \leq \widehat{K} \sqrt{\delta}$ for all $t \in[0, T]$ and $\delta \in(0,1]$.

Next, given Lemma 4.7 recall that

$$
\mathbf{P}_{t}^{\delta} \widehat{g}-\mathbf{P}_{t} \widehat{g}=\int_{0}^{t} \mathcal{L}^{\delta} \mathbf{P}_{u}^{\delta} \widehat{g} d u-\int_{0}^{t} \overline{\mathbf{P}}_{t-u} \overline{\mathcal{L}} \widehat{g} d u,
$$

from which we conclude by the finiteness of $\int_{0}^{t}\left\|\mathbf{A P}_{u} \widehat{g}\right\| d u$ and $\int_{0}^{t}\left\|\psi_{u}^{\delta}\right\| d u$, that

$$
\Delta:=\delta\left[\int_{0}^{t} \overline{\mathbf{P}}_{t-u} \psi_{u}^{\delta} d u-\rho_{t}^{\delta}\right]=\int_{0}^{t}\left(\overline{\mathbf{P}}_{t-u}-\mathbf{I}\right) \mathcal{L}^{\delta} \mathbf{P}_{u}^{\delta} \widehat{g} d u-\int_{0}^{t} \overline{\mathbf{P}}_{t-u} \overline{\mathcal{L}}\left(\mathbf{P}_{u}^{\delta} \widehat{g}-\widehat{g}\right) d u .
$$

Fixing $t \in[0, T]$ and $\delta>0$ we claim that $\Delta=\mathbf{0}$.

Indeed, fixing $h \in \mathcal{G}$, by Fubini's theorem and the definition of the adjoint semigroup $\left(\overline{\mathbf{P}}^{\star}\right)_{t \geq 0}$, clearly,

$$
\langle h \Delta\rangle_{\mu^{0}}=\int_{0}^{t}\left\langle\left(\overline{\mathbf{P}}_{t-u}^{\star} h-h\right) \mathcal{L}^{\delta} \mathbf{P}_{u}^{\delta} \widehat{g}\right\rangle_{\mu^{0}} d u-\int_{0}^{t}\left\langle\left(\overline{\mathcal{L}}^{\star} \overline{\mathbf{P}}_{v}^{\star} h\right)\left(\mathbf{P}_{t-v}^{\delta} \widehat{g}-\widehat{g}\right)\right\rangle_{\mu^{0}} d v .
$$

Further, with $h \in \mathcal{D}\left(\overline{\mathcal{L}}^{\star}\right)$ and $\widehat{g} \in \mathcal{D}\left(\mathcal{L}^{\delta}\right)$, we thus get that also

$$
\langle h \Delta\rangle_{\mu^{0}}=\int_{0}^{t}\left\langle\left(\int_{0}^{t-u} \overline{\mathbf{P}}_{v}^{\star} \overline{\mathcal{L}}^{\star} h d v\right) \mathcal{L}^{\delta} \mathbf{P}_{u}^{\delta} \widehat{g}\right\rangle_{\mu^{0}} d u-\int_{0}^{t}\left\langle\overline{\mathbf{P}}_{v}^{\star} \overline{\mathcal{L}}^{\star} h\left(\int_{0}^{t-v} \mathcal{L}^{\delta} \mathbf{P}_{u}^{\delta} \widehat{g} d u\right)\right\rangle_{\mu^{0}} d v .
$$


By Fubini's theorem, the contractiveness of $\overline{\mathbf{P}}_{v}^{\star}$ and $\mathbf{P}_{u}^{\delta}$ and the finiteness of $\left\|\overline{\mathcal{L}}^{\star} h\right\|$ and $\left\|\mathcal{L}^{\delta} \widehat{g}\right\|$, this implies that $\langle h \Delta\rangle_{\mu^{0}}=0$ for all $h \in \mathcal{G}$. So, with $\mathcal{G}$ dense in $L_{2}\left(\mu^{0}\right)$, we deduce that $\Delta=\mathbf{0}$ as claimed, i.e. $\rho_{t}^{\delta}=\int_{0}^{t} \overline{\mathbf{P}}_{t-u} \psi_{u}^{\delta} d u$.

Now, by Lemma 4.7, the contractiveness of $\overline{\mathbf{P}}_{t}$ and the convexity of $\|\cdot\|$ we have that

$$
\left\|\rho_{t}^{\delta}\right\| \leq \int_{0}^{t}\left\|\psi_{u}^{\delta}\right\| d u \leq t \widehat{K} \sqrt{\delta}
$$

thus completing the proof of the theorem.

Proof of Lemma 4.7; Fixing $f, h \in \mathcal{G}$, for each $r \in \mathcal{D}(\overline{\mathcal{L}}) \subseteq \mathcal{D}(\widetilde{\mathcal{L}})$ set $\xi(r)=$ $\frac{1}{2} \xi_{1}(r)+\xi_{2}(r)$, where

$$
\xi_{1}(r)=\left\langle f r \overline{\mathcal{L}}^{\star} h-h f \overline{\mathcal{L}} r-h r \overline{\mathcal{L}} f\right\rangle_{\mu^{0}} \quad \text { and } \quad \xi_{2}(r)=\langle h f(\widetilde{\mathcal{L}} r-\overline{\mathcal{L}} r)\rangle_{\mu^{0}} .
$$

Next for each $r \in \mathcal{D}(\overline{\mathcal{L}})$ and $\delta \in(0,1]$ let

$$
\Delta_{\delta}(r):=\delta^{-1}\left\langle h\left(\mathcal{L}^{\delta} r-\overline{\mathcal{L}} r\right)\right\rangle_{\mu^{0}}-\xi(r) .
$$

It is not hard to verify that $\xi(r)=\langle h \mathbf{A} r\rangle_{\mu^{0}}$ whenever $r \in \mathcal{D}(\mathbf{A})$. In particular, with $\mathbf{P}_{t} \widehat{g} \in \mathcal{D}(\mathbf{A})$ and $\mathbf{P}_{t}^{\delta} \widehat{g} \in \mathcal{D}(\overline{\mathcal{L}})$ this implies that

$$
\left\langle h \psi_{t}^{\delta}\right\rangle_{\mu^{0}}=\Delta_{\delta}\left(\mathbf{P}_{t}^{\delta} \widehat{g}\right)+\xi\left(\mathbf{P}_{t}^{\delta} \widehat{g}-\mathbf{P}_{t} \widehat{g}\right),
$$

for any $\widehat{g} \in \widehat{\mathcal{G}}, \delta \in(0,1]$ and $t \in[0, T]$.

To complete the proof we require the following lemmas, whose proofs are provided at the end of the section.

Lemma 4.8. Under the conditions of Theorem 4.2 there exists $K_{1}=K_{1}(f)<\infty$ such that for all $\delta \in(0,1], h \in \mathcal{G}$ and $r \in \mathcal{D}(\overline{\mathcal{L}})$

$$
\begin{aligned}
|\xi(r)| & \leq K_{1} \overline{\mathcal{E}}(r)^{1 / 2}\|h\|, \\
\left|\Delta_{\delta}(r)\right| & \leq \delta K_{1} \overline{\mathcal{E}}(r)^{1 / 2}\|h\|,
\end{aligned}
$$

where $\overline{\mathcal{E}}(r):=-\langle r \overline{\mathcal{L}} r\rangle_{\mu^{0}}$ is finite and non-negative for $r \in \mathcal{D}(\overline{\mathcal{L}})$.

Lemma 4.9. Under the conditions of Theorem 4.2. for each $T<\infty$ and $\widehat{g} \in \widehat{\mathcal{G}}$ there exists $\kappa=\kappa(f, \widehat{g}, T)<\infty$ such that for all $\delta \in(0,1]$ and any $t \in[0, T]$,

$$
\begin{aligned}
\overline{\mathcal{E}}\left(\mathbf{P}_{t}^{\delta} \widehat{g}\right) & \leq \kappa, \\
\overline{\mathcal{E}}\left(\mathbf{P}_{t}^{\delta} \widehat{g}-\mathbf{P}_{t} \widehat{g}\right) & \leq \delta \kappa .
\end{aligned}
$$

Indeed, in view of (4.7) we get from the bounds of Lemma 4.8 that

$$
\left|\left\langle h \psi_{t}^{\delta}\right\rangle_{\mu^{0}}\right| \leq K_{1}\|h\|\left[\delta \overline{\mathcal{E}}\left(\mathbf{P}_{t}^{\delta} \widehat{g}\right)^{1 / 2}+\overline{\mathcal{E}}\left(\mathbf{P}_{t}^{\delta} \widehat{g}-\mathbf{P}_{t} \widehat{g}\right)^{1 / 2}\right]
$$

Hence, by the bounds of Lemma 4.9 we deduce that $\left|\left\langle h \psi_{t}^{\delta}\right\rangle_{\mu^{0}}\right| \leq \widehat{K}\|h\| \sqrt{\delta}$ for $\widehat{K}=\widehat{K}(f, \widehat{g}, T)=2 K_{1} \sqrt{\kappa}$ finite, and all $\delta \in(0,1], t \in[0, T]$ and $h \in \mathcal{G}$. Since $\mathcal{G}$ is a dense linear subspace of $\mathcal{H}$ we conclude that $\left\|\psi_{t}^{\delta}\right\| \leq \widehat{K} \sqrt{\delta}$, as claimed.

Proof of Lemma 4.8; Fixing $f, h \in \mathcal{G}$ and $r \in \mathcal{D}(\overline{\mathcal{L}})$ we start by proving (4.8). Indeed, with $\overline{\mathcal{L}}$ and $\overline{\mathcal{L}}^{\star}$ being the generators of the strongly continuous semi-group $\overline{\mathbf{P}}_{t}$ and its adjoint $\overline{\mathbf{P}}_{t}^{\star}$, we have from (4.6) that

$$
\xi_{1}(r)=\lim _{t \rightarrow 0} \frac{1}{t}\left[\left\langle r f \overline{\mathbf{P}}_{t}^{\star} h-h f \overline{\mathbf{P}}_{t} r-h r \overline{\mathbf{P}}_{t} f+h r f\right\rangle_{\mu^{0}}\right]=\lim _{t \rightarrow 0}\left\langle h \overline{\mathbf{\Gamma}}_{t}(f, r)\right\rangle_{\mu^{0}},
$$

for the bi-linear symmetric, non-negative definite operators

$$
\overline{\boldsymbol{\Gamma}}_{t}\left(h_{1}, h_{2}\right):=t^{-1}\left[h_{1} h_{2}-h_{1} \overline{\mathbf{P}}_{t} h_{2}-h_{1} \overline{\mathbf{P}}_{t} h_{2}+\overline{\mathbf{P}}_{t} h_{1} h_{2}\right]
$$


on $\mathcal{H} \times \mathcal{H}$. Next, mimicking the arguments of (4.4) and (4.5) it follows by the $\mu^{0}$-invariance of $\overline{\mathbf{P}}_{t}$ that

$$
\limsup _{t \rightarrow 0}\left\|\overline{\boldsymbol{\Gamma}}_{t}(f, r)\right\|^{2} \leq \limsup _{t \rightarrow 0}\left\|\overline{\boldsymbol{\Gamma}}_{t}(f, f)\right\|_{\infty}\left\langle\overline{\boldsymbol{\Gamma}}_{t}(r, r)\right\rangle_{\mu^{0}}=2\|\boldsymbol{\Gamma}(f, f)\|_{\infty} \overline{\mathcal{E}}(r),
$$

for all $r \in \mathcal{D}(\overline{\mathcal{L}})$ and $f \in \mathcal{D}\left(\mathbf{P}_{t}\right)$ such that $(f, f) \in \mathcal{D}(\boldsymbol{\Gamma})$. Consequently,

$$
\left|\xi_{1}(r)\right| \leq\|h\| \limsup _{t \rightarrow 0}\left\|\overline{\boldsymbol{\Gamma}}_{t}(f, r)\right\| \leq \sqrt{2\|\boldsymbol{\Gamma}(f, f)\|_{\infty}} \overline{\mathcal{E}}(r)^{1 / 2}\|h\| .
$$

Clearly, $\left|\xi_{2}(r)\right| \leq\|h f\|\|\widetilde{\mathcal{L}} r-\overline{\mathcal{L}} r\| \leq \sqrt{K}\|f\|_{\infty}\left\|\overline{\mathcal{E}}(r)^{1 / 2}\right\| h \|$ by (4.6) and (4.1). We thus deduce that (4.8) holds for any $K_{1}(f) \geq \sqrt{\|\boldsymbol{\Gamma}(f, f)\|_{\infty}}+\sqrt{K}\|f\|_{\infty}$.

Turning to (4.9) and fixing $\delta \in(0,1]$ let $\psi_{\delta}:=\delta^{-1}\left(e^{\delta f}-\delta f-\mathbf{1}\right) \in \mathcal{D}\left(\mathbf{P}_{t}\right)$, and note that by (3.14) and (4.6)

$$
\Delta_{\delta}(r)=\widehat{\Delta}_{\delta}(r)+\left\langle h \psi_{-\delta}(\widetilde{\mathcal{L}} r-\overline{\mathcal{L}} r)\right\rangle_{\mu^{0}}
$$

where

$$
\widehat{\Delta}_{\delta}(r):=\delta^{-1}\left[\left\langle h \widetilde{\mathcal{L}}^{\delta f} r\right\rangle_{\mu^{0}}-\left\langle\left(1-e^{-\delta f}\right) h \widetilde{\mathcal{L}} r\right\rangle_{\mu^{0}}\right]-\frac{1}{2} \xi_{1}(r) .
$$

Recall that $e^{-\delta f} h \in \mathcal{G}$, so using the $\mu^{\delta f}$-symmetry of $\widetilde{\mathcal{L}}^{\delta f}$ and the $\mu^{0}$-symmetry of $\widetilde{\mathcal{L}}$, followed by the relations (3.8) and (3.13), at $g=e^{-\delta f} h$ and $g=h$, we have that

$$
\begin{aligned}
\delta\left[\widehat{\Delta}_{\delta}(r)+\frac{1}{2} \xi_{1}(r)\right] & =\left\langle r e^{\delta f} \widetilde{\mathcal{L}}^{\delta f}\left(e^{-\delta f} h\right)\right\rangle_{\mu^{0}}-\left\langle r\left[\widetilde{\mathcal{L}} h-\widetilde{\mathcal{L}}\left(e^{-\delta f} h\right)\right]\right\rangle_{\mu^{0}} \\
& =\left\langle r e^{\delta f} \widetilde{\mathcal{L}}\left(e^{-\delta f} h\right)\right\rangle_{\mu^{0}}+\frac{1}{2}\left\langle r \overline{\boldsymbol{\Gamma}}\left(e^{\delta f}, e^{-\delta f} h\right)\right\rangle_{\mu^{0}}-\langle r \widetilde{\mathcal{L}} h\rangle_{\mu^{0}} \\
& =\frac{1}{2}\left[\left\langle r e^{\delta f} \overline{\mathcal{L}}^{\star}\left(e^{-\delta f} h\right)\right\rangle_{\mu^{0}}-\left\langle r h e^{-\delta f} \overline{\mathcal{L}} e^{\delta f}\right\rangle_{\mu^{0}}-\langle h \overline{\mathcal{L}} r\rangle_{\mu^{0}}\right]
\end{aligned}
$$

Adapting the derivation of (4.12) we find that the latter expression is the limit as $t \rightarrow 0$ of $\frac{1}{2}\left\langle h e^{-\delta f} \overline{\boldsymbol{\Gamma}}_{t}\left(e^{\delta f}, r\right)\right\rangle_{\mu^{0}}$, so in view of (4.12), we deduce that

$$
\widehat{\Delta}_{\delta}(r)=\frac{1}{2} \lim _{t \rightarrow 0}\left\langle h\left[\delta^{-1} e^{-\delta f} \overline{\boldsymbol{\Gamma}}_{t}\left(e^{\delta f}, r\right)-\overline{\boldsymbol{\Gamma}}_{t}(f, r)\right]\right\rangle_{\mu^{0}} .
$$

Since $u \mapsto \overline{\boldsymbol{\Gamma}}_{t}(u, r)$ is a linear functional such that $\overline{\boldsymbol{\Gamma}}_{t}(\mathbf{1}, r)=0$ for any $r \in \mathcal{H}$, we further have that

$$
\delta^{-1} e^{-\delta f} \overline{\boldsymbol{\Gamma}}_{t}\left(e^{\delta f}, r\right)-\overline{\boldsymbol{\Gamma}}_{t}(f, r)=e^{-\delta f} \overline{\boldsymbol{\Gamma}}_{t}\left(\psi_{\delta}, r\right)-\phi_{\delta} \overline{\boldsymbol{\Gamma}}_{t}(f, r)
$$

for $\phi_{\delta}:=\mathbf{1}-e^{-\delta f}$. With $e^{-\delta f} \in[0,1]$ and $\psi_{\delta} \in \mathcal{G}$, we thus get as in the derivation of (4.13) and (4.14) that

$$
\begin{aligned}
\left\|\widehat{\Delta}_{\delta}(r)\right\| & \leq \frac{1}{2}\|h\|\left[\limsup _{t \rightarrow 0}\left\|\overline{\boldsymbol{\Gamma}}_{t}\left(\psi_{\delta}, r\right)\right\|+\left\|\phi_{\delta}\right\|_{\infty} \limsup _{t \rightarrow 0}\left\|\overline{\boldsymbol{\Gamma}}_{t}(f, r)\right\|\right] \\
& \leq k_{0}(\delta, f) \overline{\mathcal{E}}(r)^{1 / 2}\|h\|,
\end{aligned}
$$

for $k_{0}(\delta, f)=\sqrt{\left\|\boldsymbol{\Gamma}\left(\psi_{\delta}, \psi_{\delta}\right)\right\|_{\infty}}+\left\|\phi_{\delta}\right\|_{\infty} \sqrt{\|\boldsymbol{\Gamma}(f, f)\|_{\infty}}$ finite. Combining this with (4.15) and (4.1) we thus deduce that

$$
\left\|\Delta_{\delta}(r)\right\| \leq\left\|\widehat{\Delta}_{\delta}(r)\right\|+\|h\|\left\|\psi_{-\delta}\right\|_{\infty}\|\widetilde{\mathcal{L}} r-\overline{\mathcal{L}} r\| \leq k_{1}(\delta, f) \overline{\mathcal{E}}(r)^{1 / 2}\|h\|,
$$

where $k_{1}(\delta, f)=k_{0}(\delta, f)+\sqrt{K}\left\|\psi_{-\delta}\right\|_{\infty}$. Thus, we establish (4.9) once we show that $k_{1}(\delta, f) \leq \delta K_{1}(f)$ for some finite $K_{1}(f)$ and all $\delta \in(0,1]$. To this end, recall that $\left\|\phi_{\delta}\right\|_{\infty} \leq \delta\|f\|_{\infty}$ and $\left\|\psi_{-\delta}\right\|_{\infty} \leq \delta\|f\|_{\infty}^{2} / 2$ for any non-negative $f \in \mathcal{D}\left(\mathbf{P}_{t}\right)$ and $\delta>0$. Further, for any $g \in \mathcal{G}$, as $t \rightarrow 0$ the non-negative functions $\boldsymbol{\Gamma}_{t}(g, g)$ 
converge to $\boldsymbol{\Gamma}(g, g)$ with respect to the supremum norm, so it remains only to check that for any $\delta \in(0,1]$ and $t>0$,

$$
\boldsymbol{\Gamma}_{t}\left(\psi_{\delta}, \psi_{\delta}\right) \leq\left(e^{\delta\|f\|_{\infty}}-1\right)^{2} \boldsymbol{\Gamma}_{t}(f, f)
$$

(indeed, using the bound $e^{\delta u}-1 \leq \delta e^{u}$ in (4.16) results with (4.9) holding when $\left.K_{1}(f) \geq\left(e^{\|f\|_{\infty}}+\|f\|_{\infty}\right) \sqrt{\|\boldsymbol{\Gamma}(f, f)\|_{\infty}}+\sqrt{K}\|f\|_{\infty}^{2} / 2\right)$. As for (4.16), recall that for any $x, y \in \mathcal{S}$ and $\delta>0$,

$$
\left|\psi_{\delta}(y)-\psi_{\delta}(x)\right|=\left|\int_{f(x)}^{f(y)}\left(e^{\delta u}-1\right) d u\right| \leq c_{\delta}|f(y)-f(x)|,
$$

for $c_{\delta}=e^{\delta\|f\|_{\infty}}-1$. Consequently,

$\boldsymbol{\Gamma}_{t}\left(\psi_{\delta}, \psi_{\delta}\right)=t^{-1} \mathbb{E}_{x}\left[\left(\psi_{\delta}(X(t))-\psi_{\delta}(x)\right)^{2}\right] \leq c_{\delta}^{2} t^{-1} \mathbb{E}_{x}\left[(f(X(t))-f(x))^{2}\right]=c_{\delta}^{2} \boldsymbol{\Gamma}_{t}(f, f)$,

which is exactly the inequality (4.16).

Proof of Lemma 4.9; Fixing $T<\infty, f \in \mathcal{G}$ and $\widehat{g} \in \widehat{\mathcal{G}}$, recall that $(h, \widehat{g}) \in$ $\mathcal{D}(\boldsymbol{\Gamma}) \subseteq \mathcal{D}(\overline{\boldsymbol{\Gamma}})$ for any $h \in \mathcal{G}$. Consequently, then $\overline{\boldsymbol{\Gamma}}_{t}(h, \widehat{g}) \rightarrow \boldsymbol{\Gamma}(h, \widehat{g})$ as $t \rightarrow 0$. In view of (4.13) and (4.16) we see that

$$
\left\|\overline{\boldsymbol{\Gamma}}\left(\psi_{\delta}, \widehat{g}\right)\right\|^{2} \leq 2\left\|\boldsymbol{\Gamma}\left(\psi_{\delta}, \psi_{\delta}\right)\right\|_{\infty} \overline{\mathcal{E}}(\widehat{g}) \leq 2\left(e^{\delta\|f\|_{\infty}}-1\right)^{2}\|\boldsymbol{\Gamma}(f, f)\|_{\infty} \overline{\mathcal{E}}(\widehat{g})
$$

are bounded uniformly in $\delta \in(0,1]$. Further, since $e^{\delta f}=\delta \psi_{\delta}+\delta f+\mathbf{1}$,

$$
\left\|\overline{\boldsymbol{\Gamma}}\left(e^{\delta f}, \widehat{g}\right)\right\| \leq \delta\left\|\overline{\boldsymbol{\Gamma}}\left(\psi_{\delta}, \widehat{g}\right)\right\|+\delta\|\overline{\boldsymbol{\Gamma}}(f, \widehat{g})\| \leq \kappa_{0}(f) \overline{\mathcal{E}}(\widehat{g})^{1 / 2}
$$

are also bounded uniformly in $\delta \in(0,1]$ (take $\kappa_{0}(f)=e^{\|f\|_{\infty}} \sqrt{2\|\boldsymbol{\Gamma}(f, f)\|_{\infty}}$ finite).

Turning to prove (4.10), note that for any $\delta \in(0,1]$ and $r \in \mathcal{D}(\overline{\mathcal{L}})$, by (3.14),

$$
-\left\langle r \overline{\mathcal{L}}_{1}^{\delta f} r\right\rangle_{\mu^{\delta f}}=-\left\langle r \widetilde{\mathcal{L}}^{\delta f} r\right\rangle_{\mu^{\delta f}}-\langle r \overline{\mathcal{L}} r\rangle_{\mu^{0}} \geq \overline{\mathcal{E}}(r)
$$

since $\widetilde{\mathcal{L}}^{\delta f}$ is the generator of a $\mu^{\delta f}$-symmetric, strongly continuous semi-group whose domain contains $\mathcal{D}(\overline{\mathcal{L}})$, hence a negative self-adjoint operator on this set. With $\mathbf{P}_{t}^{\delta}$ a contraction for the norm of $L_{2}\left(\mu^{\delta f}\right)$ (denoted hereafter by $\|\cdot\|_{\delta}$ ), we thus find that for any $\widehat{g} \in \widehat{\mathcal{G}}$ and $t>0$,

$$
\overline{\mathcal{E}}\left(\mathbf{P}_{t}^{\delta} \widehat{g}\right) \leq-\left\langle\left(\mathbf{P}_{t}^{\delta} \widehat{g}\right)\left(\mathbf{P}_{t}^{\delta} \overline{\mathcal{L}}_{1}^{\delta f} \widehat{g}\right)\right\rangle_{\mu^{\delta f}} \leq\|\widehat{g}\|_{\delta}\left\|\overline{\mathcal{L}}_{1}^{\delta f} \widehat{g}\right\|_{\delta} .
$$

Further, $f$ is non-negative and by our assumptions, $\widehat{g}$ is in $\widehat{\mathcal{D}}_{f}$ of (3.9) where the identity (3.8) applies. We thus establish the uniform bound of (4.10) upon noting that both $\|\widehat{g}\|_{\delta} \leq e^{\|f\|_{\infty}\|\widehat{g}\|}$ and

$$
\begin{aligned}
\left\|\overline{\mathcal{L}}_{1}^{\delta f} \widehat{g}\right\|_{\delta} & \leq\left\|\widetilde{\mathcal{L}}^{\delta f} \widehat{g}\right\|_{\delta}+\left\|e^{-\delta f} \overline{\mathcal{L}} \widehat{g}\right\|_{\delta} \\
& \leq \frac{e^{\|f\|_{\infty}}}{2}\left(\|\overline{\mathcal{L}} \widehat{g}\|+\left\|\overline{\mathcal{L}}^{\star} \widehat{g}\right\|+\left\|\overline{\boldsymbol{\Gamma}}\left(e^{\delta f}, \widehat{g}\right)\right\|\right)+e^{\|f\|_{\infty}}\|\overline{\mathcal{L}} \widehat{g}\|
\end{aligned}
$$

are bounded uniformly in $\delta \in(0,1]$ (see (4.17) for the uniform bound on $\left.\left\|\boldsymbol{\Gamma}\left(e^{\delta f}, \widehat{g}\right)\right\|\right)$.

Next consider the non-negative quadratic form $\widetilde{\mathcal{E}}\left(h_{1}, h_{2}\right):=-\left\langle h_{1} \widetilde{\mathcal{L}} h_{2}\right\rangle_{\mu^{0}}$ on $\mathcal{D}(\widetilde{\mathcal{L}}) \times \mathcal{D}(\widetilde{\mathcal{L}})$, noting that $\overline{\mathcal{E}}(r)=\widetilde{\mathcal{E}}(r, r)$ for all $r \in \mathcal{D}(\overline{\mathcal{L}}) \cap \mathcal{D}\left(\overline{\mathcal{L}}^{\star}\right)$, due to (3.13). Turning to prove (4.11), by our assumptions this is the case for $r_{t}^{\delta}:=\mathbf{P}_{t}^{\delta} \widehat{g}-\mathbf{P}_{t} \widehat{g}$, hence $w_{t}^{\delta}:=\overline{\mathcal{E}}\left(r_{t}^{\delta}\right)=\widetilde{\mathcal{E}}\left(r_{t}^{\delta}, r_{t}^{\delta}\right)$. Further, $t \mapsto a_{t}^{\delta}:=\partial_{t} r_{t}^{\delta}=\mathcal{L}^{\delta} \mathbf{P}_{t}^{\delta} \widehat{g}-\overline{\mathcal{L}} \mathbf{P}_{t} \widehat{g}$ is uniformly continuous, since

$$
\sup _{|t-s| \leq \theta}\left\|a_{t}^{\delta}-a_{s}^{\delta}\right\| \leq \sup _{u \leq \theta}\left\|\left(\mathbf{P}_{u}^{\delta}-\mathbf{I}\right) \mathcal{L}^{\delta} \widehat{g}\right\|+\sup _{u \leq \theta}\left\|\left(\mathbf{P}_{u}-\mathbf{I}\right) \mathcal{L} \widehat{g}\right\|=: \varepsilon_{\delta}(\theta)
$$


which converges to zero when $\theta \downarrow 0$. With $b_{t}^{\delta}=a_{t}^{\delta}-\overline{\mathcal{L}} r_{t}^{\delta}$, we show next that

$$
\begin{aligned}
\left\|b_{t}^{\delta}\right\| & \leq 2 \delta K_{1} \sqrt{\kappa}, \\
\left\|\overline{\mathcal{L}} r_{t}^{\delta}\right\| & \leq \kappa_{1},
\end{aligned}
$$

for some finite $\kappa_{1}=\kappa_{1}(f, \widehat{g})$, the universal constants $K_{1}$ and $\kappa$ of Lemma 4.8 and (4.10), respectively, all $t \geq 0$ and $\delta \in(0,1]$. Indeed, $b_{t}^{\delta}=\mathcal{L}^{\delta} \mathbf{P}_{t}^{\delta} \widehat{g}-\overline{\mathcal{L}} \mathbf{P}_{t}^{\delta} \widehat{g}$, hence $\left\langle h b_{t}^{\delta}\right\rangle_{\mu^{0}}=\delta\left[\Delta_{\delta}\left(\mathbf{P}_{t}^{\delta} \widehat{g}\right)+\xi\left(\mathbf{P}_{t}^{\delta} \widehat{g}\right)\right]$ for any $h \in \mathcal{G}$. Thus, by Lemma 4.8 and (4.10)

$$
\left|\left\langle h b_{t}^{\delta}\right\rangle_{\mu^{0}}\right| \leq \delta K_{1}(\delta+1)\|h\| \overline{\mathcal{E}}\left(\mathbf{P}_{t}^{\delta} \widehat{g}\right)^{1 / 2} \leq \delta K_{1}(\delta+1) \sqrt{\kappa}\|h\|,
$$

and with $\mathcal{G}$ dense in $L_{2}\left(\mu^{0}\right)$ this immediately yields the bound of (4.20). Turning to prove (4.21), note that for any $t \geq 0$ and $\delta \in(0,1]$, by (4.20) the contractivity of $\mathbf{P}_{t}$ on $L_{2}\left(\mu^{0}\right)$ and the contractivity of $\mathbf{P}_{t}^{\delta}$ on $L_{2}\left(\mu^{\delta f}\right)$,

$$
\begin{aligned}
\left\|\overline{\mathcal{L}} r_{t}^{\delta}\right\| & \leq\left\|\mathcal{L}^{\delta} \mathbf{P}_{t}^{\delta} \widehat{g}\right\|+\left\|b_{t}^{\delta}\right\|+\left\|\overline{\mathcal{L}} \mathbf{P}_{t} \widehat{g}\right\| \\
& \leq e^{\|f\|_{\infty}}\left\|\mathcal{L}^{\delta} \widehat{g}\right\|_{\delta}+2 K_{1} \sqrt{\kappa}+\|\overline{\mathcal{L}} \widehat{g}\|
\end{aligned}
$$

with the right side bounded uniformly in $\delta \in(0,1]$ by some finite $\kappa_{1}=\kappa_{1}(f, \widehat{g}) \geq 1$ (see (4.18) for details).

As $\widetilde{\mathcal{L}}$ is $\mu^{0}$-symmetric, by Fubini's theorem, for any $s^{\prime} \geq s$,

$w_{s^{\prime}}^{\delta}-w_{s}^{\delta}-\left(s^{\prime}-s\right)\left[\widetilde{\mathcal{E}}\left(a_{s}^{\delta}, r_{s}^{\delta}\right)+\widetilde{\mathcal{E}}\left(a_{s^{\prime}}^{\delta}, r_{s^{\prime}}^{\delta}\right)\right]=\int_{s}^{s^{\prime}} \widetilde{\mathcal{E}}\left(a_{u}^{\delta}-a_{s}^{\delta}, r_{s}^{\delta}\right) d u-\int_{s}^{s^{\prime}} \widetilde{\mathcal{E}}\left(a_{s^{\prime}}^{\delta}-a_{u}^{\delta}, r_{s^{\prime}}^{\delta}\right) d u$.

Recall that by (4.1) and (4.21), for some universal finite constant $K$,

$$
\left\|\widetilde{\mathcal{L}} r_{t}^{\delta}\right\| \leq \sqrt{K w_{t}^{\delta}}+\left\|\overline{\mathcal{L}} r_{t}^{\delta}\right\| \leq K w_{t}^{\delta}+2 \kappa_{1},
$$

so by the uniform continuity of $t \mapsto a_{t}^{\delta}$, see (4.19),

$w_{s^{\prime}}^{\delta}-w_{s}^{\delta}-\left(s^{\prime}-s\right)\left[\widetilde{\mathcal{E}}\left(a_{s}^{\delta}, r_{s}^{\delta}\right)+\widetilde{\mathcal{E}}\left(a_{s^{\prime}}^{\delta}, r_{s^{\prime}}^{\delta}\right)\right] \leq\left(s^{\prime}-s\right) \varepsilon_{\delta}\left(s^{\prime}-s\right)\left[K w_{s^{\prime}}^{\delta}+K w_{s}^{\delta}+4 \kappa_{1}\right]$.

Further, by (4.1) also

$$
\widetilde{\mathcal{E}}\left(a_{t}^{\delta}-b_{t}^{\delta}, r_{t}^{\delta}\right)=-2\left\langle\left(\overline{\mathcal{L}} r_{t}^{\delta}\right) \widetilde{\mathcal{L}} r_{t}^{\delta}\right\rangle_{\mu^{0}} \leq\left\|\widetilde{\mathcal{L}} r_{t}^{\delta}-\overline{\mathcal{L}} r_{t}^{\delta}\right\|^{2} \leq K \overline{\mathcal{E}}\left(r_{t}^{\delta}\right)=K w_{t}^{\delta}
$$

Combining the latter pair of inequalities and the bound $\widetilde{\mathcal{E}}\left(b_{t}^{\delta}, r_{t}^{\delta}\right) \leq\left\|b_{t}^{\delta}\right\|\left\|\widetilde{\mathcal{L}} r_{t}^{\delta}\right\|$, we deduce from (4.20) and (4.22) that if $\varepsilon_{\delta}\left(s^{\prime}-s\right) \leq 2 \delta K_{1} \sqrt{\kappa}$, then

$$
w_{s^{\prime}}^{\delta}-w_{s}^{\delta} \leq\left(s^{\prime}-s\right)\left[K_{2} w_{s^{\prime}}^{\delta}+K_{2} w_{s}^{\delta}+\kappa_{2} \delta\right],
$$

for some finite universal constants $K_{2}=K_{2}\left(K_{1}, \kappa, K\right), \kappa_{2}=\kappa_{2}\left(K_{1}, \kappa, \kappa_{1}\right)$ and all $\delta \in(0,1]$. Since $w_{0}^{\delta}=0$, iterating the latter bound $n$ times, for $s^{\prime}-s=t / n$, then taking $n \rightarrow \infty$, we conclude that $w_{t}^{\delta} \leq \delta \kappa_{2} \int_{0}^{t} e^{2 K_{2} u} d u$ for all $\delta \in(0,1]$ and $t \geq 0$. That is, (4.11) holds for $\kappa(f, \widehat{g}, T)=\kappa_{2} \int_{0}^{T} e^{2 K_{2} u} d u$ finite.

\section{Pure jump processes on a Discrete state space}

We consider here pure jump processes $X(t)$ on a countable (or finite) state space $\mathcal{S}$ equipped with the discrete topology, such that the total jump rate at state $x$ is bounded uniformly over $x \in \mathcal{S}$. That is, the jump rates $c(x, y) \geq 0$ from $x$ to $y \neq x$ are such that

$$
\sup _{x \in \mathcal{S}} \sum_{y: y \neq x} c(x, y)<\infty
$$


(which trivially holds when the set $\mathcal{S}$ is finite). Recall that to each such process corresponds a strongly continuous Markov semi-group on the Banach space $\mathcal{C}_{b}$ of all bounded functions on $\mathcal{S}$, the generator of which is the bounded linear operator $\mathcal{L}: \mathcal{C}_{b} \rightarrow \mathcal{C}_{b}$ such that

$$
\mathcal{L} g(x)=\sum_{y: y \neq x} c(x, y)(g(y)-g(x)) .
$$

Conversely, any operator of the form (5.2) with non-negative $c(x, y)$ satisfying (5.1) is the generator of such a Markov process, and taking in this context $\mathcal{G}=\mathcal{C}_{b}$ eliminates all technical issues of the previous sections (about the domains of various generators). Further assuming that the process $X(\cdot)$ is irreducible, let $\mu^{0}(\cdot)$ denote its unique invariant probability measure, identified hereafter with the positive function $\mu^{0}(x):=\mu^{0}(\{x\})$ on $\mathcal{S}$. Recall that necessarily,

$$
\mu^{0}(x) \sum_{y: y \neq x} c(x, y)=\sum_{y: y \neq x} \mu^{0}(y) c(y, x), \quad \forall x \in \mathcal{S} .
$$

We proceed to compute in this case the response functions for our two generic Markov perturbations. To this end, consider the operators $\mathcal{L}^{\star}$ and $\widetilde{\mathcal{L}}=\frac{1}{2}\left(\mathcal{L}+\mathcal{L}^{\star}\right)$ of the form (5.2) but for jump rates

$$
\begin{aligned}
c^{\star}(x, y) & :=\frac{\mu^{0}(y)}{\mu^{0}(x)} c(y, x), \quad x \neq y, \\
\widetilde{c}(x, y) & :=\frac{1}{2}\left(c(x, y)+c^{\star}(x, y)\right) \quad x \neq y,
\end{aligned}
$$

respectively. By (5.3), both $c^{\star}(\cdot, \cdot)$ and $\widetilde{c}(\cdot, \cdot)$ satisfy (5.1) so $\mathcal{L}^{\star}$ and $\widetilde{\mathcal{L}}$ are both bounded operators on $\mathcal{C}_{b}$ and the generators of strongly continuous, Markov semigroups on $\mathcal{C}_{b}$, denoted $\mathbf{P}_{t}^{\star}$ and $\widetilde{\mathbf{P}}_{t}$, respectively. It is easy to check that $\mathbf{P}_{t}^{\star}$ is the adjoint of the original semi-group $\mathbf{P}_{t}$ and that $\widetilde{\mathbf{P}}_{t}$ is the $\mu^{0}$-symmetric, strongly continuous, Markov semi-group of Corollary 3.7 (both restricted to $\mathcal{C}_{b} \subseteq L_{2}\left(\mu^{0}\right)$ ).

Given a non-negative $f \in \mathcal{C}_{b}$, both Propositions 3.1 and 3.8 apply here, and their generic perturbations correspond to the bounded operators $\mathcal{L}_{0}^{f}$ and $\mathcal{L}_{1}^{f}$ on $\mathcal{C}_{b}$ having jump rates $c_{0}^{f}(x, y)=e^{-f(x)} c(x, y)$ and

$$
\begin{aligned}
c_{1}^{f}(x, y) & =\widetilde{c}(x, y)+\frac{1}{2}\left(e^{f(y)-f(x)}-1\right) c(x, y)+\frac{1}{2} e^{-f(x)}\left(c(x, y)-c^{\star}(x, y)\right) \\
& =\frac{1}{2}\left(e^{f(y)-f(x)}+e^{-f(x)}\right) c(x, y)+\frac{1}{2}\left(1-e^{-f(x)}\right) c^{\star}(x, y),
\end{aligned}
$$

respectively. Theorem 4.1 provides the response function for $X_{0}^{f}(\cdot)$ which in this case has a bounded operator $\left(\mathbf{A}_{0}\right)_{f}$ on $\mathcal{C}_{b}$ of the form

$$
\left(\mathbf{A}_{f}\right) g(x)=\sum_{y: y \neq x} a^{f}(x, y)(g(y)-g(x))
$$

with $a_{0}^{f}(x, y)=-f(x) c(x, y)$. Consider the bounded operator $\left(\mathbf{A}_{1}\right)_{f}$ of the form (5.4) with

$$
a_{1}^{f}(x, y)=a_{0}^{f}(x, y)+\frac{1}{2} f(y) c(x, y)+\frac{1}{2} f(x) c^{\star}(x, y),
$$


and let

$$
\begin{aligned}
\xi_{\delta}(x, y) & :=\delta^{-1}\left(c_{1}^{\delta f}(x, y)-c(x, y)\right)-a_{1}^{f}(x, y) \\
& =\frac{1}{2} \varphi_{-\delta}(f(x))\left[c^{\star}(x, y)-c(x, y)\right]+\frac{1}{2} \varphi_{\delta}(f(y)-f(x)) c(x, y),
\end{aligned}
$$

where $\varphi_{\delta}(r)=\delta^{-1}\left(e^{\delta r}-\delta r-1\right) \rightarrow 0$ as $\delta \rightarrow 0$, uniformly on compacts. Hence,

$$
\lim _{\delta \rightarrow 0} \sup _{x \in \mathcal{S}} \sum_{y: y \neq x}\left|\xi_{\delta}(x, y)\right|=0
$$

This in turn implies that (2.10) holds for $\mathcal{L}_{1}^{f}$ and $\left(\mathbf{A}_{1}\right)_{f}$, so by Proposition 2.9 we deduce that the Markovian perturbations $X_{1}^{f}(\cdot)$ have the response function associated with $\left(\mathbf{A}_{1}\right)_{f}$.

Suppose now that $\mathbf{A}_{f}$ is of the form (5.4) and that

$$
\sup _{x \in \mathcal{S}} \sum_{y: y \neq x}\left|a^{f}(x, y)\right|<\infty
$$

which guarantees that $\mathbf{A}_{f}$ is a bounded operator on $\mathcal{C}_{b}$. In view of Theorem 2.10, for such $\mathbf{A}_{f}$ to correspond to the response function of some perturbation $X^{f}(\cdot)$ (per Definition 2.7), it is necessary that $a^{f}(x, y)=b^{f}(x, y)-f(x) c(x, y)$, where

$$
\begin{aligned}
b^{r f}(x, y) & =r b^{f}(x, y), \quad r>0 \\
\mu^{0}(x) \sum_{y: y \neq x} b^{f}(x, y) & =\sum_{y: y \neq x} \mu^{0}(y) b^{f}(y, x), \quad \forall x \in \mathcal{S} .
\end{aligned}
$$

Slightly modifying the time change generic perturbation of Proposition 3.1 we next show that essentially these conditions on $a^{f}(x, y)$ are also sufficient for having a perturbation $X^{f}(\cdot)$ whose response function is given by (2.7).

Proposition 5.1. Suppose that the generator of the semi-group $\mathbf{P}_{t}$ of a pure jump Markov process $X(\cdot)$ on a discrete state space $\mathcal{S}$ is of the form (5.2) for jump rates $c(x, y) \geq 0$ that satisfy (5.1). To any $\mathbf{A}_{f}$ of the form (5.4) with $a^{f}(x, y)=$ $b^{f}(x, y)-f(x) c(x, y)$ satisfying (5.6) - (5.8) and such that for some $\rho^{f}<\infty$,

$$
b^{f}(x, y) \geq-\rho^{f} c(x, y) \quad \forall x \neq y,
$$

there corresponds a Markovian perturbation $X^{f}(\cdot)$ satisfying Assumption 2.5 whose response function is $R_{X^{f}, g}(s, t)=\mathbf{P}_{s} \mathbf{A}_{f} \mathbf{P}_{t-s} g$ (for $g \in \mathcal{C}_{b}$ ).

Remark 5.2. Condition (5.9) implies that $b^{f}(x, y)$ is non-negative for every $x \neq y$ such that $c(x, y)=0$ (and for a finite state space $\mathcal{S}$ it puts no other restrictions on $\left.b^{f}(x, y)\right)$.

Proof: In view of (5.7) and (5.9), if $\delta>0$ is small enough so $1-\delta \rho^{f}>0$ then

$$
\widehat{c}_{0}^{\delta f}(x, y)=e^{-\delta f(x)}\left[c(x, y)+b^{\delta f}(x, y)\right]=c_{0}^{\delta f}(x, y)+e^{-\delta f(x)} b^{\delta f}(x, y),
$$

are non-negative for all $x \neq y$. Further, by the boundedness of $f(\cdot)$, (5.1) and (5.6) we have that $\sum_{y} \widehat{c}_{0}^{\delta f}(x, y)$ is bounded, uniformly in $x \in \mathcal{S}$. Thus, there exists a pure jump Markov process $\widehat{X}_{0}^{\delta f}(\cdot)$ on $\mathcal{S}$ whose semi-group is generated by a bounded operator $\widehat{\mathcal{L}}_{0}^{\delta f}$ on $\mathcal{C}_{b}$ of the form (5.2) with the jump rates $\widehat{c}_{0}^{\delta f}(x, y)$. Moreover, it follows from (5.3), (5.8) and (5.10) that

$$
\mu^{\delta f}(x) \sum_{y: y \neq x} \widehat{c}_{0}^{\delta f}(x, y)=\sum_{y: y \neq x} \mu^{\delta f}(y) \widehat{c}_{0}^{\delta f}(y, x), \quad \forall x \in \mathcal{S}
$$


(where $\mu^{\delta f}(x)=e^{\delta f(x)} \mu^{0}(x)$ ). This implies that $\mu^{\delta f}(\cdot)$ is a finite, positive invariant measure for the semi-group of the irreducible Markov process $\widehat{X}_{0}^{\delta f}(\cdot)$, which thus satisfies Assumption 2.5 (with $\mathcal{G}=\mathcal{C}_{b}=\mathcal{B}$ ). It is easy to check that (5.5) holds for $\xi_{\delta}(x, y):=\delta^{-1}\left(\widehat{c}_{0}^{\delta f}(x, y)-c(x, y)\right)-a^{f}(x, y)$. This in turn implies that (2.10) holds in this setting, so by Proposition 2.9 we deduce that (2.6) holds as well, and that $\mathbf{A}_{f} \mathbf{P}_{s} g$ is strongly continuous on $\mathcal{C}_{b}$.

Remark 5.3. We alternatively get the response function $R_{X^{f}, g}(s, t)=\mathbf{P}_{s} \mathbf{A}_{f} \mathbf{P}_{t-s} g$ per Proposition 5.1 by adapting instead the generic perturbation of Proposition 3.8, i.e. following the same line of reasoning for the Markov perturbation $\widehat{X}_{1}^{\delta f}(\cdot)$ that correspond to the jump rates

$$
\widehat{c}_{1}^{\delta f}(x, y)=c_{1}^{\delta f}(x, y)+e^{-\delta f(x)}\left(b^{\delta f}(x, y)-\frac{\delta}{2} f(y) c(x, y)-\frac{\delta}{2} f(x) c^{\star}(x, y)\right) .
$$

Indeed, for $f(\cdot)$ non-negative and $\delta>0$ also $\psi_{\delta}=\delta^{-1}\left(e^{\delta f}-\delta f-\mathbf{1}\right)$ is non-negative, so by (5.7) and (5.9)

$$
\begin{aligned}
e^{\delta f(x)} \widehat{c}_{1}^{\delta f}(x, y) & =c(x, y)+b^{\delta f}(x, y)+\frac{\delta}{2}\left[\psi_{\delta}(y) c(x, y)+\psi_{\delta}(x) c^{\star}(x, y)\right] \\
& \geq c(x, y)+\delta b^{f}(x, y)
\end{aligned}
$$

is non-negative as soon as $1-\delta \rho^{f}>0$. With $\psi_{\delta} \in \mathcal{C}_{b}$ it follows from (5.1) and (5.6) that $\widehat{\mathcal{L}}_{1}^{\delta f}$ of the form (5.2) corresponding to jump rates $\widehat{c}_{1}^{\delta f}(x, y)$ is a bounded operator on $\mathcal{C}_{b}$ hence a generator of a semi-group for a Markov process $\widehat{X}_{1}^{\delta f}(\cdot)$. Further, from the $\mu^{0}$-invariance of $\mathcal{L}$ and $\mathbf{B}_{f}=\mathbf{A}_{f}+f \mathcal{L}$, see (5.3) and (5.8), it follows that $\left\langle\widehat{\mathcal{L}}_{1}^{\delta f} g\right\rangle_{\mu^{\delta f}}=0$ for all $g \in \mathcal{C}_{b}$ and so the irreducible Markov process $\widehat{X}_{0}^{\delta f}(\cdot)$ satisfies Assumption 2.5. Finally, with $\left\|\psi_{\delta}\right\|_{\infty} \rightarrow 0$ as $\delta \downarrow 0$ we get the stated response function upon checking that (5.5) holds for $\xi_{\delta}(x, y)=\delta^{-1}\left(\widehat{c}_{1}^{\delta f}(x, y)-\right.$ $c(x, y))-a^{f}(x, y)$.

Cycle decomposition provides a canonical construction of Markov processes on a discrete state space with a prescribed invariant measure (such as $\mu^{f}$ ). For simplicity, we consider only cycles of finite length. More precisely, equipping $\mathcal{S}$ with any complete order, let $\Gamma$ denote the collection of all finite oriented cycles $\gamma$. That is, $\gamma=\left(x_{0}, x_{1}, \ldots, x_{n}\right)$ of length $n=|\gamma| \geq 2$ is such that $x_{n}=x_{0}$ and $x_{i} \neq x_{j}$ for all $0 \leq i<j<n$. Suppose a strictly positive probability measure $\mu^{0}$ on $\mathcal{S}$ and $\alpha: \Gamma \mapsto \mathbb{R}_{+}$are such that

$$
\|\alpha\|_{\Gamma}:=\sup _{x \in \mathcal{S}} \frac{1}{\mu^{0}(x)} \sum_{\gamma: x \in \gamma}|\alpha(\gamma)|
$$

is finite (in particular, if $\mathcal{S}$ is finite then so is $\Gamma$ and $\|\alpha\|_{\Gamma}<\infty$ for any $\alpha: \Gamma \mapsto \mathbb{R}$ ). It is then easy to check that the jump rates

$$
c(x, y)=\frac{1}{\mu^{0}(x)} \sum_{\gamma \in \Gamma} \alpha(\gamma) 1_{(x, y) \in \gamma} \quad \forall x \neq y \in \mathcal{S},
$$

satisfy (5.1) and that $\mu^{0}(\cdot)$ is an invariant measure for the corresponding semigroup $\mathbf{P}_{t}$. Further, this semi-group is $\mu^{0}$-symmetric (i.e. the Markov process is 
reversible), if $\alpha(\gamma)=0$ whenever $|\gamma|>2$. Next, let $\beta^{f}: \Gamma \mapsto \mathbb{R}$ and $\alpha^{f}: \Gamma \mapsto \mathbb{R}_{+}$ for $f \in \mathcal{C}_{b}$ be such that $\left\|\beta^{f}\right\|_{\Gamma}$ is finite, $\beta^{r f}=r \beta^{f}$ and

$$
\lim _{\delta \downarrow 0}\left\|\delta^{-1}\left(\alpha^{\delta f}-\alpha\right)-\beta^{f}\right\|_{\Gamma}=0 .
$$

Then, applying once more Proposition 2.9, we deduce that the Markov process of jump rates

$$
c^{f}(x, y)=\frac{e^{-f(x)}}{\mu^{0}(x)} \sum_{\gamma \in \Gamma} \alpha^{f}(\gamma) 1_{(x, y) \in \gamma}
$$

has an invariant measure $\mu^{f}$ and the response function corresponding to

$$
a^{f}(x, y)=-f(x) c(x, y)+\frac{1}{\mu^{0}(x)} \sum_{\gamma \in \Gamma} \beta^{f}(\gamma) 1_{(x, y) \in \gamma} .
$$

We next consider some concrete examples such as the Glauber and Metropolis dynamics for Gibbs measures on finite graphs.

Example 5.4. Consider a finite graph with $\mathcal{S}$ denoting its vertexes and the symmetric $E \subseteq \mathcal{S} \times \mathcal{S}$ denoting its edges. Given $H: \mathcal{S} \mapsto \mathbb{R}$ let $\mu^{0}(x)=e^{-H(x)}$ denote the corresponding non-normalized Gibbs measure and consider the reversible Markov processes obtained by (5.11) when $\alpha(\gamma)>0$ if and only if $\gamma=(x, y, x)$ with $(x, y) \in E$. That is, having jump rates

$$
c(x, y)=e^{H(x)} \alpha(x, y) 1_{(x, y) \in E},
$$

for $\alpha(x, y)=\alpha(y, x)>0$. Two such examples are the Metropolis dynamics where $\alpha_{M}(x, y)=\min \left(e^{-H(x)}, e^{-H(y)}\right)$ and the Glauber dynamics for which $\alpha_{G}(x, y)=$ $1 /\left(e^{H(x)}+e^{H(y)}\right)$. The Markov perturbations one uses for the Metropolis (or Glauber) dynamics are of the same type as the original process, just replacing $H(\cdot)$ by $H(\cdot)-$ $\delta f(\cdot)$. Here the convergence in $\|\cdot\|_{\Gamma}$ is equivalent to a point-wise convergence on $E$ leading to the response functions that correspond to

$$
\begin{aligned}
a_{M}^{f}(x, y) & =(f(y)-f(x)) e^{-\Delta H}\left(1_{\Delta H>0}+1_{\Delta H=0} 1_{f(x)>f(y)}\right) 1_{(x, y) \in E}, \\
a_{G}^{f}(x, y) & =(f(y)-f(x)) \frac{e^{-\Delta H}}{\left(1+e^{-\Delta H}\right)^{2}} 1_{(x, y) \in E},
\end{aligned}
$$

where $\Delta H:=H(y)-H(x)$. Note in particular that while $a_{G}^{f}$ is linear in $f(\cdot)$, this is in general not the case for $a_{M}^{f}$.

\section{Finite dimensional Diffusion processes}

Here $\mathcal{S}$ is a connected, finite dimensional $\mathcal{C}^{\infty}$-manifold $M$ without boundary. We first consider compact $M$, with the treatment of non-compact $M=\mathbb{R}^{d}$ provided at the end of the section. Let $\mu^{0}$ be a probability measure on $M$ that has a smooth strictly positive density with respect to any coordinate chart for $M$ (c.f. [3, Section 6.3]). Setting $\mathcal{G}=\mathcal{C}^{\infty}(M)$ and $\Gamma(\mathbf{T}(M))$ denoting the space of smooth sections over $M$, recall that in the absence of boundary, for any $\mathbf{Z} \in \Gamma(\mathbf{T}(M))$ there exists then a unique $g_{\mathbf{Z}} \in \mathcal{G}$ such that $\mathbf{Z}^{\star} h=-\mathbf{Z} h+g_{\mathbf{Z}} h$ acts on $\mathcal{G}$ as the adjoint of $\mathbf{Z}$ with respect to the inner product of $L_{2}\left(\mu^{0}\right)$ (and $g_{\mathbf{Z}}=\mathbf{Z}^{\star} \mathbf{1}$ ). For $\mathbf{D}_{i} \in \Gamma(\mathbf{T}(M)$ ), $i=0,1, \ldots, d$, consider the operator $\mathcal{L}=\sum_{i=1}^{d} \mathbf{D}_{i} \circ \mathbf{D}_{i}+\mathbf{D}_{0}$ on $\mathcal{G}$ (that is, a 
diffusion generator in the Hörmander form, see [13]). Any such operator can be rewritten as

$$
\mathcal{L}=-\sum_{i=1}^{d} \mathbf{D}_{i}^{\star} \circ \mathbf{D}_{i}+\mathbf{Y},
$$

where $\mathbf{Y}=\mathbf{D}_{0}+\sum_{i=1}^{d} g_{\mathbf{D}_{i}} \mathbf{D}_{i}$ is also in $\Gamma(\mathbf{T}(M))$. Note that such operator is the restriction to $\mathcal{G}$ of the generator of a strongly continuous Markov semi-group $\mathbf{P}_{t}$ on $\mathcal{C}_{b}=\mathcal{C}_{b}(\mathcal{S})$ such that $\widehat{\mathcal{G}}=\mathcal{G}=\mathcal{C}^{\infty}(M)$ (see for example, [3, Theorem 6.3.2]). In particular, the corresponding Markov process $X(\cdot)$ can be constructed as the unique solution of a certain Stratonovich stochastic differential equation (S-SDE), c.f. [3, Exercise 6.3.22].

Also, the semi-group $\mathbf{P}_{t}$ is $\mu^{0}$-invariant if and only if $g_{\mathbf{Y}}=\mathbf{0}$ (see 3, Theorem 6.3.2]). In particular, if $\left\{\mathbf{D}_{1}, \ldots, \mathbf{D}_{d}\right\}$ satisfies Hörmander's strong hypo-elliptic condition (i.e. (H) of [3] Section 6.3]), then for any $\mathbf{D}_{0} \in \Gamma(\mathbf{T}(M))$ there exists a unique probability measure $\mu^{0}$ (of a smooth strictly positive density with respect to any coordinate chart) for which $g_{\mathbf{Y}}=\mathbf{0}$. We next provide the diffusion process $X_{1}^{f}(\cdot)$ of the generalized Langevin dynamics of Proposition 3.8 and its response function.

Proposition 6.1. Suppose $g_{\mathbf{Y}}=\mathbf{0}$ and for any non-negative $f \in \mathcal{C}^{\infty}(M)$ let $\mathbf{Y}^{f}=e^{-f} \mathbf{Y}+\sum_{i=1}^{d}\left(\mathbf{D}_{i} f\right) \mathbf{D}_{i}$. Then,

$$
\mathcal{L}_{1}^{f}=-\sum_{i=1}^{d} \mathbf{D}_{i}^{\star} \circ \mathbf{D}_{i}+\mathbf{Y}^{f},
$$

is (the restriction to $\mathcal{G}=\mathcal{C}^{\infty}(M)$ of) the generator of a strongly continuous, $\mu^{f}$ invariant Markovian semi-group $\left(\mathbf{P}_{1}^{f}\right)_{t}$ on $\mathcal{C}_{b}$ with $\mathcal{G}$ closed under the action of this semi-group (and the Markov process $X_{1}^{f}(\cdot)$ is the unique solution of a certain $S$-SDE). If further

$$
\int_{M}|\mathbf{Y} g|^{2} d \mu^{0} \leq K \sum_{i=1}^{d} \int_{M}\left|\mathbf{D}_{i} g\right|^{2} d \mu^{0},
$$

for some $K<\infty$ and all $g \in \mathcal{G}$, then $X_{1}^{f}(\cdot)$ has a response function that corresponds to $\left(\mathbf{A}_{1}\right)_{f}=\sum_{i=1}^{d}\left(\mathbf{D}_{i} f\right) \mathbf{D}_{i}-f \mathbf{Y}$, a linear operator of domain $\mathcal{G}$.

Remark 6.2. The process $X_{1}^{f}(\cdot)$ is a diffusion on $M$ that differs from $X(\cdot)$ only by the addition of a smooth drift term corresponding to $\mathbf{Y}^{f}-\mathbf{Y}$. We note in passing that $X(\cdot)$ is reversible (i.e. has a $\mu^{0}$-symmetric semi-group) if and only if $\mathbf{Y}=\mathbf{0}$, in which case the added drift is of a gradient form (and $X_{1}^{f}(\cdot)$ is known in the literature as the Langevin dynamic).

Remark 6.3. If $\mathbf{D}_{0}=\sum_{i=1}^{d} h_{i} \mathbf{D}_{i}$ for some $h_{i} \in L_{2}\left(\mu^{0}\right)$, then Girsanov transformation shows that the laws of $X_{1}^{f}(\cdot)$ and $X(\cdot)$ are mutually absolutely continuous on $\mathcal{C}([0, T] ; M)$ for each $T<\infty$. The Langevin dynamic is in this respect more natural that the time change generic perturbation (of generator $\mathcal{L}_{0}=e^{-f} \mathcal{L}$ ), for which this is of course not the case. If in addition $h_{i} \in L_{\infty}\left(\mu^{0}\right)$ for $i=1, \ldots, d$ (as for example, in case of uniform ellipticity), then the condition (6.1) is trivially satisfied. 
Proof: Our assumption that $g_{\mathbf{Y}}=\mathbf{0}$ means that $\mathbf{Y}^{\star}=-\mathbf{Y}$. Hence, acting on $\mathcal{G}$, the adjoint

$$
\mathcal{L}^{\star}=-\sum_{i=1}^{d} \mathbf{D}_{i}^{\star} \circ \mathbf{D}_{i}-\mathbf{Y}
$$

of $\mathcal{L}$ with respect to the inner product of $L_{2}\left(\mu^{0}\right)$ is such that $\mathcal{L}^{\star} \mathcal{G} \subseteq \mathcal{G}$. It is thus the restriction to $\mathcal{G}$ (and a core) of the generator of the adjoint semi-group $\mathbf{P}_{t}^{\star}$ on $\mathcal{C}_{b}$, with $\mathcal{G}$ closed under the action of $\mathbf{P}_{t}^{\star}$. Consequently, the generator of the $\mu^{0}$-symmetric semi-group $\widetilde{\mathbf{P}}_{t}$ of Corollary 3.7 is just $\widetilde{\mathcal{L}}=-\sum_{i=1}^{d} \mathbf{D}_{i}^{\star} \circ \mathbf{D}_{i}$ (when acting on $\mathcal{G}$ ). Moreover, here

$$
\boldsymbol{\Gamma}(f, g)=2 \sum_{i=1}^{d}\left(\mathbf{D}_{i} f\right)\left(\mathbf{D}_{i} g\right)
$$

satisfies the Leibniz rule $\boldsymbol{\Gamma}(f h, g)=h \boldsymbol{\Gamma}(f, g)+f \boldsymbol{\Gamma}(h, g)$, so $\boldsymbol{\Gamma}\left(e^{f}, g\right)=e^{f} \boldsymbol{\Gamma}(f, g)$ and for any non-negative $f \in \mathcal{G}$, the generator of the $\mu^{f}$-symmetric semi-group $\widetilde{\mathbf{P}}_{t}^{f}$ of Lemma 3.5 is such that for $g \in \mathcal{G}$,

$$
\widetilde{\mathcal{L}}^{f} g=-\left(1-e^{-f}\right) \sum_{i=1}^{d} \mathbf{D}_{i}^{\star} \circ \mathbf{D}_{i} g+\sum_{i=1}^{d}\left(\mathbf{D}_{i} f\right)\left(\mathbf{D}_{i} g\right) .
$$

It follows that when acting on $\mathcal{G}$ the generator $\mathcal{L}_{1}^{f}=\widetilde{\mathcal{L}}^{f}+e^{-f} \mathcal{L}$ we use in Proposition 3.8 is merely

$$
\mathcal{L}_{1}^{f}=-\sum_{i=1}^{d} \mathbf{D}_{i}^{\star} \circ \mathbf{D}_{i}+\mathbf{Y}^{f} .
$$

It is easy to check that for any $\mathbf{Z} \in \Gamma(\mathbf{T}(M))$, the operator $\mathbf{Z}^{\star, f}=-\mathbf{Z}+\left(g_{\mathbf{Z}}-\mathbf{Z} f\right)$ acts on $\mathcal{G}$ as the adjoint of $\mathbf{Z}$ with respect to the inner product of $L_{2}\left(\mu^{f}\right)$. Further, with $\mathbf{Y} e^{-f}=-e^{-f} \mathbf{Y} f$, it follows that

$$
g_{e^{-f} \mathbf{Y}}-e^{-f} \mathbf{Y} f=e^{-f} g_{\mathbf{Y}}=\mathbf{0} .
$$

Thus, $\mathcal{L}_{1}^{f}=-\sum_{i=1}^{d} \mathbf{D}_{i}^{\star, f} \circ \mathbf{D}_{i}+e^{-f} \mathbf{Y}$, and $\left(e^{-f} \mathbf{Y}\right)^{\star, f}=-\left(e^{-f} \mathbf{Y}\right)$. Hence, by [3, Theorem 6.3.2], now with respect to the finite measure $\mu^{f}$ on $M$ that is also of a smooth strictly positive density, we find that $\mathcal{L}_{1}^{f}$ is the generator of a strongly continuous, $\mu^{f}$-invariant Markovian semi-group $\left(\mathbf{P}_{1}^{f}\right)_{t}$ on $\mathcal{C}_{b}$ such that $\mathcal{G}$ is closed under its action, with $X_{1}^{f}(\cdot)$ characterized as the unique strong solution of some S-SDE (this direct construction bypasses that of Proposition 3.8).

Carefully examining the proof of Theorem 4.2 one verifies that there is no need to ever consider functions outside $\mathcal{G}$ in case this algebra is in the domain of the generators $\mathcal{L}, \mathcal{L}^{*}, \widetilde{\mathcal{L}}, \mathcal{L}_{1}^{\delta f}$ and is closed under the action of the corresponding Markovian semi-groups on $\mathcal{C}_{b}$. It then suffices to define the operator $\left(\mathbf{A}_{1}\right)_{f}$ of (4.2) only on $\mathcal{G}$ and since here $\mathcal{L}-\widetilde{\mathcal{L}}=\mathbf{Y}$ we deduce from (6.2) that $\left(\mathbf{A}_{1}\right)_{f} g=\sum_{i=1}^{d}\left(\mathbf{D}_{i} f\right)\left(\mathbf{D}_{i} g\right)-f \mathbf{Y} g$ for all $g \in \mathcal{G}$, as stated. Further, it follows that in such a situation $X_{1}^{f}(\cdot)$ has the response function corresponding to $\left(\mathbf{A}_{1}\right)_{f}$ as soon as $\|\widetilde{\mathcal{L}} g-\mathcal{L} g\|^{2} \leq-K\langle g \mathcal{L} g\rangle_{\mu^{0}}$ for some $K<\infty$ and all $g \in \mathcal{G}$, which is exactly our condition (6.1).

Consider next the non-compact manifold $M=\mathbb{R}^{d}$, denoting by $\mathcal{C}^{\infty}, \mathcal{C}_{b}^{\infty}, \mathcal{C}_{0}^{\infty}$ the collections of smooth functions, smooth functions with bounded derivatives of all orders, smooth functions of compact support on $M=\mathbb{R}^{d}$, respectively. Let $\mathcal{G}$ 
be the vector space spanned by $\mathbf{1}$ and the collection of Schwartz test functions on $M$ (i.e. functions in $\mathcal{G}$ are elements of $\mathcal{C}^{\infty}$ whose derivatives of all positive orders decay faster than any power of $\|x\|$ ), and consider the Markovian semi-group $\mathbf{P}_{t}$ on $\mathcal{G}$ that is generated by $\mathcal{L}=\sum_{i=1}^{d} \mathbf{D}_{i} \circ \mathbf{D}_{i}+\mathbf{D}_{0}$ where for $i=0, \ldots, d$,

$$
\mathbf{D}_{i}=\sum_{k=1}^{d} a_{i, k} \frac{\partial}{\partial x_{k}}
$$

with $a_{i, k} \in \mathcal{C}_{b}^{\infty}$ for $i \geq 1$ while $a_{0, k} \in \mathcal{C}^{\infty}$ with $\frac{\partial a_{0, k}}{\partial x_{j}} \in \mathcal{C}_{b}^{\infty}$ for $k, j=1, \ldots, d$ (that is, the drift of our diffusion may be unbounded, but its derivatives are bounded). Hence,

$$
\mathcal{L}=\sum_{j, k=1}^{d} c_{j, k} \frac{\partial^{2}}{\partial x_{j} \partial x_{k}}+\sum_{j=1}^{d} b_{j} \frac{\partial}{\partial x_{j}},
$$

where for each $x \in M$,

$$
c_{j, k}=\sum_{i=1}^{d} a_{i, j} a_{i, k}, \quad b_{j}=a_{0, j}+\sum_{i, k=1}^{d} a_{i, k} \frac{\partial a_{i, j}}{\partial x_{k}} .
$$

Suppose that $\left\{\mathbf{D}_{1}, \ldots, \mathbf{D}_{d}\right\}$ satisfies Hörmander's strong hypo-elliptic condition and there exists a bounded below Lyapunov function $V \in \mathcal{C}^{\infty}$, such that $\mathcal{L} V \leq 0$ and

$$
\lim _{\|x\| \rightarrow \infty} V(x)=\infty .
$$

This implies that the diffusion has a unique invariant measure $\mu^{0}$ with a strictly positive smooth density $\rho \in \mathcal{C}_{b}^{\infty}$ with respect to Lebesgue's measure on $M$ (see [6]). In view of [18, Theorem 3.14] the semi-group $\mathbf{P}_{t}$ maps $\mathcal{G}$ into itself (more precisely they require bounded drift, but under our assumptions, for $\mathbf{D}_{0}$ of linear growth the transition probability function has sub-Gaussian tails and once this is shown, a localization argument reduces to the case covered in [18. An alternative approach is to use that fact that if $g \in \mathcal{G}$ then $\mathcal{L} \mathbf{P}_{t} g=\mathbf{P}_{t}(\mathcal{L} g)$, where $\mathcal{L} g \in \mathcal{G}$ and use the weighted Sobolev norm estimates of [7, Theorem 4.1]).

Now take $f \in \mathcal{C}_{0}^{\infty}$ and upon making the relevant modifications, apply Proposition 6.1 in this setting.

\section{Stochastic SPIN Systems}

We consider next systems of locally interacting diffusion processes, indexed by the $d$-dimensional lattice $\mathbb{Z}^{d}$. Such processes naturally arise in statistical physics, where all Gibbs states of the interaction potential are invariant measures for the chosen dynamics. In particular, in the presence of a phase transition we typically have non-uniqueness of the Gibbs state and infinitely many invariant measures for the Markov process $X(\cdot)$. Note that in contrast with the setting of Section 6 , here the state space $\mathcal{S}$ is such that we typically do not have an obvious dense algebra of test functions $\mathcal{G}$ in $\mathcal{D}\left(\mathbf{P}_{t}\right)$ which is closed under the action of the semi-group $\mathbf{P}_{t}$ (for example, the algebra of functions depending on finitely many coordinates is typically not closed under action of $\mathbf{P}_{t}$ ).

For simplicity we restrict ourselves to pair interaction potentials and consider first the simpler case of compact spin spaces $\mathcal{S}=\left(\mathbf{S}^{1}\right)^{\mathbb{Z}^{d}}$, with spins taking values in the one dimensional torus $\mathbf{S}^{1}$ (equipped with Lebesgue measure and its $\sigma$-algebra 
$\mathcal{S}_{1}$ ), and having smooth, symmetric, finite range interactions. Specifically, for $\mathbf{x}=$ $\left(x_{i}, i \in \mathbb{Z}^{d}\right)$ and any $V \subset \subset \mathbb{Z}^{d}$ (i.e. $V$ finite), consider the Hamiltonian

$$
H_{V}(\mathbf{x})=\sum_{i \in V} \Phi_{i}\left(x_{i}\right)+\frac{1}{2} \sum_{i \neq j \in V} \Phi_{i, j}\left(x_{i}, x_{j}\right)+\sum_{i \in V, j \notin V} \Phi_{i, j}\left(x_{i}, x_{j}\right),
$$

where the potentials $\Phi_{i} \in \mathcal{C}^{\infty}\left(\mathbf{S}^{1}\right)$ and $\Phi_{i, j} \in \mathcal{C}^{\infty}\left(\mathbf{S}^{1} \times \mathbf{S}^{1}\right), i \neq j$ are such that $\Phi_{i, j}(x, y)=\Phi_{j, i}(x, y)=\Phi_{i, j}(y, x)$ and $\Phi_{i, j}=\mathbf{0}$ if $|i-j|>r$. Let

$$
H_{i}(\mathbf{x}):=H_{\{i\}}(\mathbf{x})=\Phi_{i}\left(x_{i}\right)+\sum_{j \in N(i)} \Phi_{i, j}\left(x_{i}, x_{j}\right),
$$

where $N(i)=\left\{j \in \mathbb{Z}^{d}: 1<|i-j| \leq r\right\}$ denotes the $r$-neighborhood of $i$, excluding $i$. Given smooth functions $\Psi_{i}(\mathbf{x})=\Psi_{i}\left(x_{j}, j \in N(i)\right) \in \mathcal{C}^{\infty}\left(\left(\mathbf{S}^{1}\right)^{N(i)}\right)$, set

$$
b_{i}(\mathbf{x})=-\Phi_{i}^{\prime}\left(x_{i}\right)-\sum_{j \in N(i)} \Phi_{i, j}^{\prime}\left(x_{i}, x_{j}\right)+\Psi_{i}(\mathbf{x}) e^{H_{i}(\mathbf{x})},
$$

where $\Phi_{i}^{\prime}(x)=\partial_{x} \Phi_{i}(x), \Phi_{i, j}^{\prime}(x, y)=\partial_{x} \Phi_{i, j}(x, y)$ (and hereafter $\partial_{x}$ denotes the smooth section corresponding to this differential operator on the $\mathcal{C}^{\infty}$-manifold $\mathbf{S}^{1}$ ). We assume that

$$
\begin{gathered}
\sup _{i}\left|\Phi_{i}^{\prime}(0)\right| \leq C, \quad \sup _{i, j}\left|\Phi_{i, j}^{\prime}(0,0)\right| \leq C, \\
\inf _{i} \inf _{x} \Phi_{i}^{\prime \prime}(x) \geq-K, \quad \inf _{i, j} \inf _{x, y} \Phi_{i, j}^{\prime \prime}(x, y) \geq-K \\
\sup _{x, y}\left|\partial_{x} \partial_{y} \Phi_{i, j}(x, y)\right| \leq c(i-j), \\
\sup _{i}\left|\Psi_{i}(\mathbf{0})\right| \leq C, \quad \sup _{i} \sup _{j \in N(i)} \sup _{\mathbf{x}}\left|\partial_{x_{j}} \Psi_{i}(\mathbf{x})\right| \leq C
\end{gathered}
$$

for some finite constants $C, K$ and $c(k)$. Under these conditions there exists a strong Markov process $X(t)$ on $\mathcal{S}=\left(\mathbf{S}^{1}\right)^{\mathbb{Z}^{d}}$ (equipped with the corresponding product topology), which is the unique strong solution of the system of stochastic differential equations

$$
d X_{i}(t)=b_{i}(X(t)) d t+\sqrt{2} d W_{i}(t), \quad i \in \mathbb{Z}^{d},
$$

where $W_{i}(t), i \in \mathbb{Z}^{d}$ are independent Brownian motions on $\mathbf{S}^{1}$ (c.f. 23]).

We note in passing that with $\mathbf{S}^{1}$ compact, conditions (7.2)-(7.5) trivially hold for example whenever $i \mapsto \Phi_{i}, i \mapsto \Psi_{i}$ and $(i, j) \mapsto \Phi_{i, j}$ are translation invariant (and also in some other cases).

Let $\mathcal{G}$ denote the dense subset of $\mathcal{C}_{b}(\mathcal{S})$, consisting of all local smooth functions. That is, $\mathcal{G}=\left\{\mathcal{C}^{\infty}\left(\left(\mathbf{S}^{1}\right)^{V}\right)\right.$ for some $\left.V \subset \subset \mathbb{Z}^{d}\right\}$. Restricted to the algebra $\mathcal{G}$, the generator of the corresponding semi-group $\mathbf{P}_{t}$ on $\mathcal{C}_{b}(\mathcal{S})$ takes the form

$$
\mathcal{L}=\sum_{i}\left(\mathbf{D}_{i} \circ \mathbf{D}_{i}+b_{i} \mathbf{D}_{i}\right)
$$

where $\mathbf{D}_{i} g=\partial_{x_{i}} g$. Hence, $\mathcal{L} g \in \mathcal{G}$ for each $g \in \mathcal{G}$.

The solution $X(t)$ of (7.6) is smooth with respect to $X(0)=\mathbf{x}$ with derivatives $Y_{i j}(t)=\partial_{x_{j}} X_{i}(t)$ such that $Y_{i j}(t)=\sum_{k} \int_{0}^{t} B_{i k}(X(s)) Y_{k j}(s) d s$ and $Y_{i j}(0)=1_{j}(i)$, where by our assumptions $B_{i k}=\mathbf{D}_{k} b_{i}$ are uniformly bounded and the sum is over the finite support of the local function $b_{i}$. While $\mathbf{P}_{t} g$ is not necessarily local for all 
$g \in \mathcal{G}$ (so $\mathcal{G}$ is not closed under the action of this semi-group), nevertheless by the chain rule $\mathbf{D}_{i} \mathbf{P}_{t} g=\sum_{k} \mathbb{E}_{\mathbf{x}}\left[\mathbf{D}_{k} g(X(t)) Y_{i k}(t)\right]$, which is thus uniformly bounded in $\mathbf{x}$ (c.f. the proof of [4, Theorem 2.2] or [12] which further shows the existence of a smooth transition probability density). Further, iterating this procedure to deal with the second derivatives, one easily verifies that for all $\widehat{g} \in \widehat{\mathcal{G}}$,

$$
\sum_{i}\left\|\mathbf{D}_{i} \circ \mathbf{D}_{i} \widehat{g}\right\|_{\infty}+\sum_{i}\left\|b_{i} \mathbf{D}_{i} \widehat{g}\right\|_{\infty}<\infty .
$$

In particular, $h \mathbf{P}_{t} g \in \mathcal{D}(\mathcal{L})$ for all $h, g \in \mathcal{G}$ and the form (7.7) of $\mathcal{L}$ extends to any such function.

Under our assumptions the collection $\mathbf{G}(\Phi)$ of Gibbs measures associated with the potentials $\left\{\Phi_{i}, \Phi_{i, j}, i, j \in \mathbb{Z}^{d}\right\}$ is non-empty (see [12]). That is, $\mu \in \mathbf{G}(\Phi)$ if and only if the DLR equations $\int_{B} \mu_{V}(d \mathbf{y} \mid \mathbf{x}) \mu(d \mathbf{x})=\mu(B)$ hold for all $B \in \mathcal{S}_{1}^{\mathbb{Z}^{d}}$ and $V \subset \subset \mathbb{Z}^{d}$, where the probability measure $\mu_{V}(\cdot \mid \mathbf{x})$ on $\left(\mathbf{S}^{1}\right)^{V}$ has the density

$$
\mu_{V}(\mathbf{y} \mid \mathbf{x})=\frac{1}{Z_{V}(\mathbf{x})} \exp \left(-H_{V}(\mathbf{y})\right) \prod_{i \notin V} \delta_{x_{i}}\left(y_{i}\right),
$$

with respect to Lebesgue measure on this set (and $Z_{V}(\mathbf{x}) \in \mathbb{R}_{+}$is the corresponding normalizing constant). Since $\mathbf{S}^{1}$ has no boundary and $\partial_{x_{i}} \Psi_{i}=\mathbf{0}$, considering the form (7.7) and the DLR relation for $V=\{i\}$, it is not hard to verify that $\langle\mathcal{L} g\rangle_{\mu}=0$ for any $\mu \in \mathbf{G}(\Phi)$ and $g \in \widehat{\mathcal{G}}$. That is, any measure in $\mathbf{G}(\Phi)$ is invariant for $X(t)$. Further, fixing $\mu^{0} \in \mathbf{G}(\Phi)$, upon using the explicit form of the local specification $\mu_{\{i\}}(\cdot \mid \mathbf{x})$ and integration by part, it is easy to check that for all $g, h \in \mathcal{D}\left(\mathbf{D}_{i}\right)$ and for $\mu^{0}$-a.e. $\mathbf{x} \in \mathcal{S}$,

$$
\left\langle h \mathbf{D}_{i} g\right\rangle_{\mu_{\{i\}}(\cdot \mid \mathbf{x})}=\left\langle g\left(H_{i}^{\prime} h-\mathbf{D}_{i} h\right)\right\rangle_{\mu_{\{i\}}(\cdot \mid \mathbf{x})}
$$

where $H_{i}^{\prime}=\mathbf{D}_{i} H_{i}=\Phi_{i}^{\prime}\left(x_{i}\right)+\sum_{j \in N(i)} \Phi_{i, j}^{\prime}\left(x_{i}, x_{j}\right)$ is in $\mathcal{G}$. That is, the operator $\mathbf{D}_{i}^{\star}=-\mathbf{D}_{i}+H_{i}^{\prime}$, having the same domain as $\mathbf{D}_{i}$, is the adjoint of $\mathbf{D}_{i}$ in $L_{2}\left(\mu^{0}\right)$. In particular,

$$
\overline{\mathcal{L}}=\sum_{i}\left(-\mathbf{D}_{i}^{\star} \circ \mathbf{D}_{i}+\Psi_{i} e^{H_{i}} \mathbf{D}_{i}\right)
$$

with its adjoint in $L_{2}\left(\mu^{0}\right)$

$$
\overline{\mathcal{L}}^{\star}=\sum_{i}\left(-\mathbf{D}_{i}^{\star} \circ \mathbf{D}_{i}-\Psi_{i} e^{H_{i}} \mathbf{D}_{i}\right)
$$

having the same domain as $\overline{\mathcal{L}}$ and such that $\overline{\mathcal{L}}^{\star} \mathcal{G} \subseteq \mathcal{G}$. Consequently, the same applies for the self-adjoint operator $\widetilde{\mathcal{L}}=-\sum_{i} \mathbf{D}_{i}^{\star} \circ \mathbf{D}_{i}$ (whose domain thus contains that of $\overline{\mathcal{L}})$.

When $\Psi_{i}=\mathbf{0}$ for all $i$, the Markov process $X(t)$ is merely the usual reversible Langevin dynamic and $\mathbf{G}(\Phi)$ is then precisely the collection of measures for which its semi-group $\mathbf{P}_{t}$ is symmetric (see [23, Theorem 4.3]). In this case, it is further known that for $d=1,2$ there are no other invariant measures for $\mathbf{P}_{t}$ (c.f. [12]).

Fixing a non-negative, smooth, local function $f \in \mathcal{C}^{\infty}\left(\left(\mathbf{S}^{1}\right)^{U}\right)$ for some $U \subset \subset \mathbb{Z}^{d}$ (with $f \neq \mathbf{0}$ ), and $\mu^{0} \in \mathbf{G}(\Phi)$, the probability measure $\bar{\mu}^{f}=Z_{f}^{-1} e^{f} \mu^{0}$ (with $\left.Z_{f}=\left\langle e^{f}\right\rangle_{\mu^{0}}\right)$ is a Gibbs measure corresponding to potentials $\left\{\Phi_{i}, \Phi_{i, j}, i, j \in \mathbb{Z}^{d}\right.$ and $\left.\widetilde{\Phi}_{U}=-f\right\}$. Consequently, $\bar{\mu}^{f}$ is invariant for the strong Markov process $X^{f}(t)$ 
which is the unique strong solution of the SDE (17.6) with drift

$$
b_{i}^{f}=-H_{i}^{\prime}+\mathbf{D}_{i} f+\Psi_{i}^{f} \exp \left(H_{i}-f 1_{U}(i)\right),
$$

provided $\Psi_{i}^{f} \in \mathcal{C}^{\infty}\left(\left(\mathbf{S}^{1}\right)^{N(i)}\right)$ satisfy (7.5). For example, when taking $\Psi_{i}^{f}=\mathbf{0}$ the generator of the corresponding semi-group coincides with $\widetilde{\mathcal{L}}^{f}+e^{-f} \widetilde{\mathcal{L}}$ of Lemma 3.5 and Corollary 3.7, at least when acting on smooth functions satisfying (7.8) (and in particular, on $\widehat{\mathcal{G}}$ ).

We now seek $\Psi_{i}^{f}$ for which this Markovian perturbation has a response function. To this end, suppose first that $\Psi_{i}=\mathbf{0}$ for all but finitely many $i \in \mathbb{Z}^{d}$. In this case we may and shall enlarge $U$ so $\Psi_{i}=\mathbf{0}$ for all $i \notin U$ in which case taking $\Psi_{i}^{f}=\Psi_{i}$ results effectively with the Markovian perturbation $X_{1}^{f}(\cdot)$ of Proposition 3.8 . The same argument we used to derive (7.9) also shows that $\left\langle r \Psi_{i} e^{H_{i}} \mathbf{D}_{i} r\right\rangle_{\mu_{\{i\}}(\cdot \mid \mathbf{x})}=0$ for $\mu^{0}$-a.e. $\mathbf{x} \in \mathcal{S}$ and any $r \in \mathcal{D}\left(\mathbf{D}_{i}\right)$. Consequently, for any $r \in \mathcal{D}(\overline{\mathcal{L}})$,

$$
\langle r \overline{\mathcal{L}} r\rangle_{\mu^{0}}=\langle r \widetilde{\mathcal{L}} r\rangle_{\mu^{0}}=-\sum_{i}\left\|\mathbf{D}_{i} r\right\|^{2}
$$

and applying Cauchy-Schwarz inequality we see that the condition 4.1 holds here for $K=\sum_{i \in U}\left\|\Psi_{i} e^{H_{i}}\right\|^{2}$ finite. In view of Remark 4.4 we can apply Theorem 4.2 and conclude that this Markovian perturbation yields a response function (in the sense of Definition 2.77), that corresponds to

$$
\mathbf{A}_{f}=\sum_{i}\left(\mathbf{D}_{i} f\right) \mathbf{D}_{i}-f \sum_{i \in U} \Psi_{i} e^{H_{i}} \mathbf{D}_{i} .
$$

Whereas the condition (4.1) typically fails to hold when $\Psi_{i}$ is non-zero at infinitely many sites $i \in \mathbb{Z}^{d}$, the perturbed drift $b_{i}^{f}$ with $\Psi_{i}^{f}=\Psi_{i}$ (where now $U=U_{f}$ remains the domain of $f$ ), can be directly shown to still yield the response function corresponding to the operator $\mathbf{A}_{f}$ of (7.10). While we do not detail this argument, note that we merely replaced the non-symmetric part of the perturbation (namely, $\left.\left(1-e^{-f}\right)(\overline{\mathcal{L}} r-\widetilde{\mathcal{L}} r)\right)$, by the "localized" non-symmetric operator $\left(1-e^{-f}\right) \sum_{i \in U} \Psi_{i} e^{H_{i}} \mathbf{D}_{i} r$, which as we have seen, is dominated by $-\langle r \overline{\mathcal{L}} r\rangle_{\mu^{0}}$ (and we can handle the latter as in the proof of Theorem 4.2).

Considering next the case of unbounded spins, where $\mathbf{S}^{1}$ is replaced by $\mathbb{R}$, we restrict the state space $\mathcal{S}$ to the subset of tempered configurations $\mathbf{x} \in \mathbb{R}^{\mathbb{Z}^{d}}$ such that $\sum_{i}\left|x_{i}\right|^{2}(1+|i|)^{-2 p}$ is finite for $p$ large enough. Considering only the usual Langevin dynamics where $\Psi_{i}=\mathbf{0}$ and assuming once more that (7.2)-(7.4) hold, guarantees the existence of a strong Markov process $X(t)$ with state space $\mathcal{S}$ which is the unique strong solution of the SDE (7.6), see [23. In this setting we take

$$
\mathcal{G}=\left\{g \in \mathcal{C}^{\infty}\left(\mathbb{R}^{V}\right), V \subset \subset \mathbb{Z}^{d}, \partial_{x_{i}} g \text { has a compact support in } \mathbb{R}^{V}, \forall i \in V\right\},
$$

where again (7.7) holds and $\mathcal{L G} \subseteq \mathcal{G}$.

Generally $\mathbf{P}_{t} g$ is neither a local function, nor having derivatives of compact support, so once again $\mathcal{G}$ is not closed under the action of $\mathbf{P}_{t}$. Nevertheless, under suitable assumptions (7.8) holds, the process $X(t)$ has invariant (Gibbs) measures and the $\bar{\mu}^{f}$-symmetric Markovian semi-group associated with the perturbed drift $b_{i}^{f}$ yields the same explicit response function as before. For example, as shown in [23], the set $\mathbf{G}(\Phi)$ of Gibbs measures is non-empty when for some $b>\sum_{k} c(k)$

$$
\sup _{x, i}\left(b x^{2}-x \Phi_{i}^{\prime}(x)\right)<\infty .
$$


Acknowledgment We are grateful to Leticia Cugliandolo whose notes on the derivation of the FDT for reversible Markov chains on a finite state space motivated this work and to Yves Le Jan for his help with the proof of Proposition 3.1. We also benefited from valuable feedback of Jorge Kurchan, Joseph Avron, Joel Lebowitz and Andrea Montanari about the role and use of the FDT in physics, from a comment of Ben Goldys regarding the setting for a non-compact state space, and from the anonymous referees input which greatly improved the presentation of our work.

\section{REFERENCES}

[1] D. Bakry, and M. Emery, Diffusions hypercontractives, in Séminaire de probabilités XIX, LNM 1123, Springer, (1985), 179-206.

[2] N. Bouleau, and F. Hirsch, Dirichlet forms and analysis on Wiener space, de Gruyter, New York, (1991).

[3] J. D. Deuschel and D. W. Stroock, Large Deviations, American Mathematical Society; Reprint edition, 2000.

[4] J. D. Deuschel and D. W. Stroock, Hypercontractivity and spectral gap of symmetric diffusions with applications to the stochastic Ising models, J. Funct. Anal., 92, (1990), 30-48.

[5] N. Dunford, and J. T. Schwartz, Linear operators, part I: general theory, Interscience, New-York, (1958).

[6] R. Durrett, Stochastic calculus: a practical introduction, CRC Press, (1996).

[7] Eckmann J.-P and Hairer M, Spectral Properties of Hypoelliptic Operators Commn.Math.Phys, 235, (2003), 233-257.

[8] A. Einstein, On the motion of small particles suspended in liquids at rest required by the molecular-kinetic theory of heat, Ann. Physics (Leipzig), 17, (1905), 549-560.

[9] M. Fukushima, Y. Oshima and M. Takeda, Dirichlet forms and symmetric Markov processes, de Gruyter, New York, (1994).

[10] J. A. Goldstein, Semigroups of linear operators and applications, Oxford Univ. press, (1985).

[11] T. Hanney and M. R. Evans, Einstein relation for nonequilibrium steady states, J. Stat. Phys. 111, (2003), 1377-1390.

[12] R. Holley and D. Stroock, Diffusions on the infinite dimensional torus, J. Func. Anal. 42, (1981), 29-63.

[13] L. Hörmander, Hypoelliptic second order differential equations, Acta Math. 119, (1967), $147-171$.

[14] M. Ichiyanagi, Differential transport coefficients and the fluctuation-dissipation theorem for non-equilibrium steady states, Physica A, 201, (1993), 626-648.

[15] D.-Q. Jiang, M. Qian and M.-P. Qian, Mathematical theory of nonequilibrium steady states, Lecture notes in mathematics 1833, Springer-Verlag, New-York (2004).

[16] R. Kubo, The fluctuation-dissipation theorem, Rep. Prog. Phys. 29, (1966), 255-284.

[17] R. Kubo, M. Toda and N. Hashitsume, Statistical physics II, second edition, SpringerVerlag (1991).

[18] S. Kusuoka and D.W. Stroock, Application of the Malliavin calculus, II, J. Fac. Sci. Univ. Tokyo IA Math. 32 (1985), 1-76.

[19] J. L. Lebowitz and H. Rost, The Einstein relation for the displacement of a test particle in a random environment, Stoch. Proc. and Appl. 54, (1994), 183-196.

[20] M. Loulakis, Mobility and Einstein relation for a tagged particle in asymmetric mean zero random walk with simple exclusion, Ann. Inst. H. Poinc. Probab. Statist. 41, (2005), $237-254$.

[21] Z.-M. Ma and M. Röckner, Introduction to the the theory of (non-symmetric) Dirichlet forms, Springer-Verlag (1991).

[22] H. Nyquist, Thermal agitation of electric charge in conductors, Phys. Review, 32, (1928), 110-113.

[23] T. Shiga and A. Shimizu, Infinite dimensional stochastic differential equations and their applications, J. Math. Kyoto Univ., 20, (1980), 395-416. 